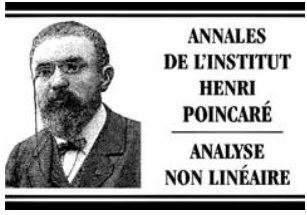

www.elsevier.com/locate/anihpc

\title{
Weakly stable multidimensional shocks
}

\section{Chocs multidimensionnels faiblement stables}

\author{
Jean-François Coulombel \\ UMPA, CNRS-UMR 5669, École normale supérieure de Lyon, 46, allée d'Italie, 69364 Lyon cedex 07, France
}

Received 17 September 2002; received in revised form 6 January 2003; accepted 15 April 2003

Available online 4 October 2003

\begin{abstract}
We study the linear stability of multidimensional shock waves for systems of conservation laws in the case where Majda's uniform stability condition is violated. The linearized problem is attacked using the "good unknown" of Alinhac. We prove an energy estimate and show that the solutions to the linearized problem have singularities localized along bicharacteristic curves originating from the boundary. The application to isentropic gas dynamics is detailed.
\end{abstract}

(C) 2004 L'Association Publications de l'Institut Henri Poincaré. Published by Elsevier B.V. All rights reserved

Résumé

Nous étudions dans cet article la stabilité linéaire des ondes de choc multidimensionnelles qui ne sont pas uniformément stables au sens de Majda. Pour le problème linéarisé, nous montrons une estimation d'énergie sur la «bonne inconnue» d'Alinhac. En particulier, nous montrons que les solutions du problème linéarisé ont des singularités le long de courbes bicaractéristiques émanant du bord du domaine. Pour finir, nous traitons l'exemple de la dynamique des gaz isentropique.

(C) 2004 L'Association Publications de l'Institut Henri Poincaré. Published by Elsevier B.V. All rights reserved

MSC: 35L67; 35L50; 35L65; 76L05

Keywords: Conservation laws; Multidimensional shocks; Stability; Symmetrizers

\section{Introduction}

In [24] and [23], Majda proved the existence of multidimensional shock waves for hyperbolic systems of conservation laws. The analysis relied on a uniform stability assumption. However, previous works [5,12] have exhibited some examples where the uniform stability condition breaks down. In [12], we have begun to extend Majda's linear analysis to these particular examples, namely we have proved an energy estimate on a constant coefficients linearized system. Here we adopt a general approach and prove a complete linear stability result for a class of shock waves that are not uniformly stable. The analysis is closely related to what was done in [12].

E-mail address: jfcoulom@umpa.ens-lyon.fr (J.-F. Coulombel). 
To avoid any possible confusion, we shall not include the case of nonclassical shock waves in this work though this field has known a significant increase of interest over the past few years, see, e.g., $[5,6,15,16]$ and the references therein.

We shall focus in this paper on multidimensional hyperbolic systems: the one-dimensional case is far different from the multidimensional case since shock waves are either uniformly stable or violently unstable, see [25]. The scalar case is also known to be very different from the system case since scalar conservation laws provide us with a unified theory of existence and uniqueness of solutions in the large, see, e.g., [13,33].

We consider a system of $N$ conservation laws in time-space $\mathbb{R} \times \mathbb{R}^{d}$ :

$$
\sum_{j=0}^{d} \partial_{j} f_{j}(u)=0
$$

where $x_{0}$ is the time variable, also denoted by $t$ in the sequel, $\left(x_{1}, \ldots, x_{d}\right)$ is the space variable and $\partial_{j}$ stands for the partial derivative with respect to $x_{j}$. The fluxes $f_{0}, \ldots, f_{d}$ are $\mathcal{C}^{\infty}$ functions defined on an open set $U$ of $\mathbb{R}^{N}$ with values in $\mathbb{R}^{N}$. The jacobian matrix of $f_{j}$ at a point $u \in U$ will be denoted by $A_{j}(u)$.

We assume that the system (1) does not consist of a single conservation law (in one or several space variables), that is, $N \geqslant 2$. We also assume that the space dimension is $d \geqslant 2$ (see the preceeding remarks). We first assume that (1) is a symmetric hyperbolic system of conservation laws:

Assumption 1. There exists a $\mathcal{C}^{\infty}$ mapping $\Sigma: U \rightarrow \mathcal{M}_{N}(\mathbb{R})$ such that

$\forall j=0, \ldots, d \forall u \in U \Sigma(u) A_{j}(u)$ is symmetric,

$\forall K$ compact $\subset U \exists c_{K}>0$ such that $\Sigma(u) A_{0}(u) \geqslant c_{K} I$ for all $u \in K$.

Recall that Assumption 1 is satisfied when there exists a strictly convex entropy, see [13,33]. Assumption 1 is met by many physical examples such as Euler equations of gas dynamics, Maxwell equations or the wave equation. Moreover, Assumption 1 is the key tool to solve the Cauchy problem associated with (1) for smooth initial data (namely in a Sobolev space of large index), see [25,33].

Because the system has been assumed to be symmetric hyperbolic, the matrix $A(u, \xi)$ defined by the formula:

$$
\forall \xi \in \mathbb{R}^{d}, \quad A(u, \xi):=A_{0}(u)^{-1} \sum_{j=1}^{d} \xi_{j} A_{j}(u)
$$

is diagonalizable over $\mathbb{R}$ for all state $u \in U$ and all wave vector $\xi \in \mathbb{R}^{d}$ (see [33]). However, we shall need a little more than hyperbolicity to carry out the study of the linear stability of shock waves. In [21], the system was assumed to be strictly hyperbolic but it has been shown in [24] that a suitable "block structure condition" (that is met by strictly hyperbolic systems) is sufficient to carry out the study of initial boundary value problems and the study of the linear stability of shock waves, see also $[11,27,29]$. The block structure condition will be recalled further in this paper. In [26], Métivier has shown that the block structure condition was met by every hyperbolic system with constant multiplicity. We are thus naturally led to make the assumption that (1) is a system with constant multiplicity:

Assumption 2. There exist $\mathcal{C}^{\infty}$ real valued mappings $\lambda_{1}, \ldots, \lambda_{q}$ defined on $U \times \mathbb{R}^{d} \backslash\{0\}$, and fixed integers $m_{1}, \ldots, m_{q}$ such that the $\lambda_{j}$ 's are the eigenvalues, with multiplicity $m_{j}$, of the matrix $A(u, \xi)$ defined by (2). Furthermore, the $\lambda_{j}$ 's satisfy

$$
\forall u \in U, \forall \xi \in \mathbb{R}^{d} \backslash\{0\}, \quad \lambda_{1}(u, \xi)<\cdots<\lambda_{q}(u, \xi) .
$$

We point out that Assumption 2 is easily checked on the system. However, one could replace Assumption 2 by the more abstract block structure condition, as was made in [24,27,29]. 
Example. Consider Euler's equations of isentropic gas dynamics in space dimension $d$ :

$$
\left\{\begin{array}{l}
\partial_{t} \rho+\nabla \cdot(\rho \mathbf{u})=0 \\
\partial_{t}(\rho \mathbf{u})+\nabla \cdot(\rho \mathbf{u} \otimes \mathbf{u})+\nabla p=0
\end{array}\right.
$$

where $\rho$ stands for the density of the fluid, $\mathbf{u}$ for the velocity, $p$ for the pressure. Quantities $\rho$ and $p$ are linked by an equation of state $p=p(\rho)$. In the domain $\{\rho>0\}$, hyperbolicity (we mean Assumption 1) amounts to requiring that the pressure satisfies

$$
c^{2}:=\frac{\mathrm{d} p}{\mathrm{~d} \rho}>0 .
$$

As usual, $c$ denotes the sound speed in the fluid. Under this condition on the pressure law, Euler's equations are endowed with a strictly convex entropy, so Assumption 1 is met. Moreover, the eigenvalues of the corresponding matrix $A(U, \xi), U=(\rho, \mathbf{u})$, are given by

$$
\begin{array}{ll}
\lambda_{1}(U, \xi)=\mathbf{u} \cdot \xi-c|\xi| & \text { with multiplicity } m_{1}=1, \\
\lambda_{2}(U, \xi)=\mathbf{u} \cdot \xi & \text { with multiplicity } m_{2}=d-1, \\
\lambda_{3}(U, \xi)=\mathbf{u} \cdot \xi+c|\xi| & \text { with multiplicity } m_{3}=1
\end{array}
$$

and therefore Assumption 2 is met. We shall detail in Section 4 how the general analysis of this paper applies in the context of isentropic gas dynamics.

Note that Lundquist's equations of magnetohydrodynamics violate Assumption 2. The study of shock waves in MHD is a very intricate subject due to the appearance of many "pathologies" (nonconstant multiplicity, occurrence of under- and over-compressive shocks, etc.). We refer to [9] and to the references therein for some results on this subject.

Because of the natural development of singularities in finite time, see [3], it appears natural to seek solutions to (1) as functions that are smooth on either side of a hypersurface of $\mathbb{R} \times \mathbb{R}^{d}$. Recall the following classical result:

Proposition 1.1. Let $\Gamma=\left\{x_{d}-\varphi\left(x_{0}, \ldots, x_{d-1}\right)=0\right\}$ be a smooth hypersurface in $\mathbb{R} \times \mathbb{R}^{d}$, and let $u$ be a smooth function on either side of $\Gamma$. Then $u$ is a weak solution of (1) if and only if $u$ satisfies (1) (in the classical sense) on either side of $\Gamma$ and if the Rankine-Hugoniot conditions hold at each point of $\Gamma$ :

$$
\forall \mathbf{x}=\left(x_{0}, \ldots, x_{d}\right) \in \Gamma, \quad \sum_{j=0}^{d-1} \partial_{j} \varphi\left[f_{j}(u)\right](\mathbf{x})-\left[f_{d}(u)\right](\mathbf{x})=0,
$$

the partial derivatives of $\varphi$ in the above formula being evaluated at $\left(x_{0}, \ldots, x_{d-1}\right)$. In (3), we have let $\left[f_{j}(u)\right](\mathbf{x})$ denote the jump of the quantity $f_{j}(u)$ across the hypersurface:

$$
\left[f_{j}(u)\right](\mathbf{x}):=\lim _{s \rightarrow 0^{+}}\left(f_{j}(u(\mathbf{x}+s \mathbf{n}))-f_{j}(u(\mathbf{x}-s \mathbf{n}))\right) \text { with } \mathbf{n}=\left(-\partial_{0} \varphi, \ldots,-\partial_{d-1} \varphi, 1\right) .
$$

The existence of such a piecewise smooth solution to (1) is a free boundary problem since the function $\varphi$ defining the hypersurface $\Gamma$ is part of the unknown of the problem. To overcome this first difficulty, we begin by straightening the variables in order to work in a fixed domain: given a smooth function $\varphi$ on $\mathbb{R}^{d}$, we define a change of variables in $\mathbb{R}^{d+1}$ by the formula:

$$
\Phi\left(x_{0}, \ldots, x_{d}\right):=\left(x_{0}, \ldots, x_{d-1}, x_{d}+\varphi\left(x_{0}, \ldots, x_{d-1}\right)\right) .
$$

We have chosen here the standard change of variables (as in [24,27,29]): it maps the hyperplane $\left\{x_{d}=0\right\}$ onto the hypersurface $\Gamma$ and the two half-spaces $\left\{ \pm x_{d}>0\right\}$ on the two sides of $\Gamma$. Other choices for the change of variables 
(that may be appropriate for characteristic problems) may be found in [14]. We now perform a change of unknown functions. If $u$ is a smooth function on either side of $\Gamma$, then the function $u_{\sharp}$ defined by

$$
\forall\left(x_{0}, \ldots, x_{d}\right) \in \mathbb{R}^{d+1}, \quad u_{\sharp}\left(x_{0}, \ldots, x_{d}\right):=u\left(\Phi\left(x_{0}, \ldots, x_{d}\right)\right)
$$

is smooth on either side of the hyperplane $\left\{x_{d}=0\right\}$. Denoting by $u_{\sharp}^{+}$(respectively $u_{\sharp}^{-}$) the restriction of $u_{\sharp}$ to the half-space $\left\{x_{d}>0\right\}$ (respectively $\left\{x_{d}<0\right\}$ ), Proposition 1.1 asserts that $u$ is a weak solution of (1) if and only if

$$
\begin{cases}L\left(u_{\sharp}^{ \pm}, \varphi\right) u_{\sharp}^{ \pm}=0, & \text { if } \pm x_{d}>0, \\ B\left(u_{\sharp}^{+}, u_{\sharp}^{-}, \varphi\right)=0, & \text { if } x_{d}=0,\end{cases}
$$

where operators $L$ and $B$ are defined by the following formulas:

$$
L(v, \psi) w:=\sum_{j=0}^{d-1} A_{j}(v) \partial_{j} w+\widetilde{A_{d}}(v, \nabla \psi) \partial_{d} w
$$

with

$$
\begin{aligned}
& \widetilde{A_{d}}(v, \nabla \psi):=A_{d}(v)-\sum_{j=0}^{d-1} \partial_{j} \psi A_{j}(v), \\
& B\left(w^{+}, w^{-}, \psi\right):=\sum_{j=0}^{d-1} \partial_{j} \psi\left[f_{j}(w)\right]-\left[f_{d}(w)\right] .
\end{aligned}
$$

Now that the domain is fix, the problem reduces to the following question: given an initial datum $u^{0}$ that is smooth on either side of a hypersurface $\left\{x_{d}=\varphi^{0}\left(x_{1}, \ldots, x_{d-1}\right)\right\}$, does there exist a solution $\left(u_{\sharp}, \varphi\right)$ of (4) with initial value $\left(u_{\sharp}^{0}, \varphi^{0}\right)$, at least locally in time? This question has received a positive answer in [23] under the socalled uniform stability condition (we shall recall it in Section 2), see [25,34] for a description of the method. The main idea is that Eqs. (5a)-(5c) are satisfied for planar shocks and the linear uniform stability of these trivial solutions implies the existence of nontrivial solutions. As detailed in $[5,12,35]$, the uniform stability condition breaks down in some cases and Majda's nonlinear existence result can not be applied anymore. Our purpose is therefore to derive a linear stability result under a weaker condition than Majda's one.

\section{The constant coefficients analysis}

We first examine the linear stability of a planar shock in order to formulate our "weak stability" assumptions. A planar shock is a solution of (1) that takes the form

$$
\mathbf{u}= \begin{cases}\mathbf{u}_{r} & \text { if } x_{d}>\sigma t+v \cdot y, \\ \mathbf{u}_{l} & \text { if } x_{d}<\sigma t+v \cdot y\end{cases}
$$

where $\mathbf{u}_{r}$ and $\mathbf{u}_{l}$ are fixed vectors belonging to the open set $U, y=\left(x_{1}, \ldots, x_{d-1}\right)$ is the vector formed by the tangential space coordinates, $v$ is a vector in $\mathbb{R}^{d-1}$ and $\sigma$ is the normal speed of propagation of the front. This corresponds to the equation

$$
\varphi\left(x_{0}, \ldots, x_{d-1}\right)=\sigma x_{0}+\sum_{j=1}^{d-1} v_{j} x_{j}
$$

for the shock front. We easily check that $\mathbf{u}$ is a solution of (1) if and only if the Rankine-Hugoniot relations

$$
\sigma\left[f_{0}(\mathbf{u})\right]+\sum_{j=1}^{d-1} v_{j}\left[f_{j}(\mathbf{u})\right]=\left[f_{d}(\mathbf{u})\right]
$$




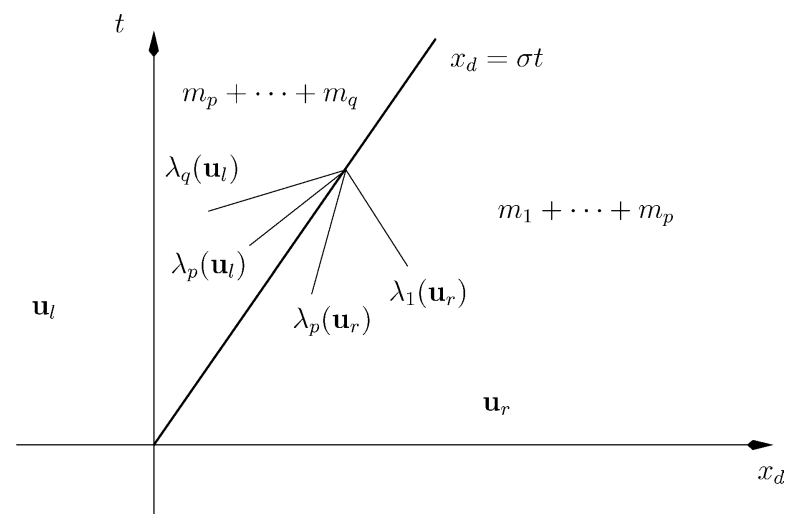

Fig. 1. Characteristics entering the shock front.

are satisfied. Performing a rotation of the axis, we may assume $v=0$. Changing last space variable $x_{d}$ into $x_{d}-\sigma t$, we may also assume that the shock is stationary, that is, $\sigma=0$. Note that Assumption 2 is still satisfied after this change of observer.

Following Lax [22], we assume that $\mathbf{u}$ is a $p$-shock:

Assumption 3. There exists an integer $p \in\{1, \ldots, q\}$ such that the following inequalities hold:

$$
\lambda_{p-1}\left(\mathbf{u}_{l}, e_{d}\right)<0<\lambda_{p}\left(\mathbf{u}_{l}, e_{d}\right) \quad \text { and } \quad \lambda_{p}\left(\mathbf{u}_{r}, e_{d}\right)<0<\lambda_{p+1}\left(\mathbf{u}_{r}, e_{d}\right),
$$

where $e_{d}:=(0, \ldots, 0,1) \in \mathbb{R}^{d}$ is the direction of propagation of the shock $\mathbf{u}$. Moreover, $\lambda_{p}$ is a simple eigenvalue, that is, $m_{p}=1$.

In the case $p=1$ (respectively $p=q$ ), that is, in the case of an extreme shock, the first inequality on the left (respectively the last on the right) is ignored. Recall that Assumption 3 is made in order to avoid under- (or over-) determinacy of the boundary value problem (4). In view of the number of jump conditions, the number of characteristics (counted with their multiplicity) entering the shock front curve has to be equal to $N+1$, see Fig. 1 . Recall also that if $\lambda_{p}$ is a multiple eigenvalue, that is, $m_{p}>1$, the $p$-th field is linearly degenerate by Boillat's Theorem, see [33]. This is a second reason why we assume that $\lambda_{p}$ is a simple eigenvalue.

Remark. Applying the implicit functions theorem, we easily see that the set of solutions $\left(\sigma, v, u_{l}, u_{r}\right)$ of the Rankine-Hugoniot relations (7) consists, in the neighborhood of $\left(0,0, \mathbf{u}_{l}, \mathbf{u}_{r}\right)$ of all vectors of the form

$$
\left(\sigma, v, u_{l}, h\left(\sigma, v, u_{l}\right)\right)
$$

where $h$ is a $\mathcal{C}^{\infty}$ mapping defined on a neighborhood of $\left(0,0, \mathbf{u}_{l}\right) \in \mathbb{R} \times \mathbb{R}^{d-1} \times U$ and satisfies $h\left(0,0, \mathbf{u}_{l}\right)=\mathbf{u}_{r}$. Moreover, planar shock waves that are close to our reference planar shock wave $\mathbf{u}$ are $p$-shocks, that is, meet Assumption 3.

In what follows, we base most of our analysis on the reference shock wave $\mathbf{u}$ but we shall also need to deal with shock waves that are close to $\mathbf{u}$. These shock waves share the main properties of $\mathbf{u}$.

\subsection{The weak stability condition}

We now introduce the linearized operators around the shock $\mathbf{u}$ : consider a family $u_{s}=\mathbf{u}+s v$ and $\varphi_{s}=s \psi$. Then we define 


$$
\begin{aligned}
\mathcal{L}_{\mathbf{u}}\left(v^{ \pm}, \psi\right) & :=\left.\frac{\mathrm{d}}{\mathrm{d} s} L\left(u_{s}^{ \pm}, \varphi_{s}\right) u_{s}^{ \pm}\right|_{s=0}, \\
\mathcal{B}_{\mathbf{u}}(v, \psi) & :=\left.\frac{\mathrm{d}}{\mathrm{d} s} B\left(u_{s}^{+}, u_{s}^{-}, \varphi_{s}\right)\right|_{s=0} .
\end{aligned}
$$

Since $\mathbf{u}$ is constant on either side of $\left\{x_{d}=0\right\}$, the linearized operators read

$$
\begin{aligned}
& \mathcal{L}_{\mathbf{u}}\left(v^{ \pm}, \psi\right)=\mathcal{L}_{\mathbf{u}} v^{ \pm}=\sum_{j=0}^{d} A_{j}\left(\mathbf{u}_{r, l}\right) \partial_{j} v^{ \pm}, \quad \pm x_{d}>0, \\
& \mathcal{B}_{\mathbf{u}}(v, \psi)=\sum_{j=0}^{d-1} \partial_{j} \psi\left[f_{j}(\mathbf{u})\right]-A_{d}\left(\mathbf{u}_{r}\right) v^{+}+A_{d}\left(\mathbf{u}_{l}\right) v^{-}, \quad x_{d}=0 .
\end{aligned}
$$

We let $b_{j}(\mathbf{u}):=\left[f_{j}(\mathbf{u})\right]($ for $j=0, \ldots, d-1)$ and

$$
M(\mathbf{u})\left(\begin{array}{c}
v^{+} \\
v^{-}
\end{array}\right):=-A_{d}\left(\mathbf{u}_{r}\right) v^{+}+A_{d}\left(\mathbf{u}_{l}\right) v^{-} .
$$

Then the linearized boundary value operator $\mathcal{B}_{\mathbf{u}}$ reads

$$
\mathcal{B}_{\mathbf{u}}(v, \psi)=\sum_{j=0}^{d-1} \partial_{j} \psi b_{j}(\mathbf{u})+M(\mathbf{u})\left(\begin{array}{c}
v^{+} \\
v^{-}
\end{array}\right) .
$$

We are interested in the following boundary value problem for the unknown functions $(v, \psi)$ :

$$
\begin{cases}\mathcal{L}_{\mathbf{u}} v^{ \pm}=f_{ \pm} & \text {for } \pm x_{d}>0 \\ \mathcal{B}_{\mathbf{u}}(v, \psi)=g & \text { for } x_{d}=0\end{cases}
$$

where $f_{ \pm}$and $g$ are source terms.

Note that system (9) is a constant coefficients hyperbolic boundary value problem; moreover, the boundary $\left\{x_{d}=0\right\}$ is noncharacteristic because of Assumption 3: both matrices $A_{d}\left(\mathbf{u}_{r}\right)$ and $A_{d}\left(\mathbf{u}_{l}\right)$ are regular. Kreiss' theory [21] does not apply directly because partial derivatives of $\psi$ are involved in the boundary conditions. Nonetheless, we attack problem (9) by the same kind of arguments: formally, we perform a Laplace transform in $x_{0}$ and a Fourier transform in the tangential space variables $\left(x_{1}, \ldots, x_{d-1}\right)$. We also make the change of variables $\left(x_{d} \rightarrow-x_{d}\right)$ in the evolution equation for $v^{-}$. This yields the following system of ODEs:

$$
\begin{aligned}
& \left(\tau A_{0}\left(\mathbf{u}_{r}\right)+\mathrm{i} \sum_{j=1}^{d-1} \eta_{j} A_{j}\left(\mathbf{u}_{r}\right)\right) V^{+}+A_{d}\left(\mathbf{u}_{r}\right) \frac{\mathrm{d} V^{+}}{\mathrm{d} x_{d}}=\widehat{F_{+}}, \\
& \left(\tau A_{0}\left(\mathbf{u}_{l}\right)+\mathrm{i} \sum_{j=1}^{d-1} \eta_{j} A_{j}\left(\mathbf{u}_{l}\right)\right) V^{-}-A_{d}\left(\mathbf{u}_{l}\right) \frac{\mathrm{d} V^{-}}{\mathrm{d} x_{d}}=\widehat{F_{-}},
\end{aligned}
$$

in the domain $\left\{x_{d}>0\right\}$, with the boundary conditions:

$$
\left(\tau b_{0}(\mathbf{u})+\mathrm{i} \sum_{j=1}^{d-1} \eta_{j} b_{j}(\mathbf{u})\right) \Psi+M(\mathbf{u})\left(\begin{array}{c}
V^{+}(0) \\
V^{-}(0)
\end{array}\right)=\widehat{G},
$$

on $\left\{x_{d}=0\right\}$. The complex number $\tau=\gamma+\mathrm{i} \eta_{0}$ has nonnegative real part and $\left(\eta_{1}, \ldots, \eta_{d-1}\right)$ is a wave vector in $\mathbb{R}^{d-1}$. In the sequel, we shall denote by $\eta$ the vector $\left(\eta_{0}, \eta_{1}, \ldots, \eta_{d-1}\right) \in \mathbb{R}^{d}$.

Because the boundary is noncharacteristic, we may rewrite (10a), (10b) as an ODEs system of the form

$$
\frac{\mathrm{d}}{\mathrm{d} x_{d}}\left(\begin{array}{l}
V^{+} \\
V^{-}
\end{array}\right)=\mathcal{A}(\mathbf{u}, \eta, \gamma)\left(\begin{array}{l}
V^{+} \\
V^{-}
\end{array}\right)+\mathcal{A}_{d}(\mathbf{u})^{-1}\left(\begin{array}{c}
\widehat{F_{+}} \\
\widehat{F_{-}}
\end{array}\right)
$$


with

$$
\mathcal{A}(\mathbf{u}, \eta, \gamma):=\left(\begin{array}{cc}
\mathcal{A}_{r}(\mathbf{u}, \eta, \gamma) & \mathbf{0} \\
\mathbf{0} & \mathcal{A}_{l}(\mathbf{u}, \eta, \gamma)
\end{array}\right) .
$$

Matrices $\mathcal{A}_{r, l}$ and $\mathcal{A}_{d}$ in (12) are defined by

$$
\begin{aligned}
& \mathcal{A}_{r, l}(\mathbf{u}, \eta, \gamma):=\mp A_{d}\left(\mathbf{u}_{r, l}\right)^{-1}\left(\gamma A_{0}\left(\mathbf{u}_{r, l}\right)+\mathrm{i} \sum_{j=0}^{d-1} \eta_{j} A_{j}\left(\mathbf{u}_{r, l}\right)\right), \\
& \mathcal{A}_{d}(\mathbf{u}):=\left(\begin{array}{cc}
A_{d}\left(\mathbf{u}_{r}\right) & \mathbf{0} \\
\mathbf{0} & -A_{d}\left(\mathbf{u}_{l}\right)
\end{array}\right) .
\end{aligned}
$$

Defining

$$
\mathcal{A}_{j}(\mathbf{u}):=\left(\begin{array}{cc}
A_{j}\left(\mathbf{u}_{r}\right) & \mathbf{0} \\
\mathbf{0} & A_{j}\left(\mathbf{u}_{l}\right)
\end{array}\right), \quad 0 \leqslant j \leqslant d-1,
$$

we easily find

$$
\mathcal{A}(\mathbf{u}, \eta, \gamma)=-\mathcal{A}_{d}(\mathbf{u})^{-1}\left(\gamma \mathcal{A}_{0}(\mathbf{u})+\mathrm{i} \sum_{j=0}^{d-1} \eta_{j} \mathcal{A}_{j}(\mathbf{u})\right) .
$$

We also define the symbol associated with the shock front:

$$
b(\mathbf{u}, \eta, \gamma):=\gamma b_{0}(\mathbf{u})+\mathrm{i} \sum_{j=0}^{d-1} \eta_{j} b_{j}(\mathbf{u}) .
$$

As pointed out by Hersh [17], the homogeneous part of the ODEs (12) is hyperbolic when $\gamma>0$, that is, the matrix $\mathcal{A}$ has no purely imaginary eigenmodes when $\gamma>0$. For $\gamma>0$, we define $\mathcal{E}^{-}(\mathbf{u}, \eta, \gamma)$ as the stable subspace of $\mathcal{A}$, which is the set of initial values of solutions to (12) that are square integrable on $\left[0,+\infty\left[\right.\right.$ when $\widehat{F_{+}}=\widehat{F_{-}}=0$. Because of the decoupled nature of (12), the stable subspace $\mathcal{E}^{-}(\mathbf{u}, \eta, \gamma)$ is nothing but the product of the stable subspaces of $\mathcal{A}_{r}$ and $\mathcal{A}_{l}$. In the case of an extreme shock, one of these two stable subspaces is trivial. The dimension of $\mathcal{E}^{-}(\mathbf{u}, \eta, \gamma)$ is equal to the dimension of $\mathcal{E}^{-}(\mathbf{u}, 0, \gamma)$ for all $\eta$ (this is just a continuity/connectedness argument). To compute the dimension of $\mathcal{E}^{-}(\mathbf{u}, 0, \gamma)$, we seek the eigenmodes of $\mathcal{A}(\mathbf{u}, 0, \gamma)$. These are the roots $\omega$ of the dispersion equation

$$
\operatorname{det}[\omega I-\mathcal{A}(\mathbf{u}, 0, \gamma)]=0 .
$$

The definition of $\mathcal{A}$ shows that $\omega I-\mathcal{A}(\mathbf{u}, 0, \gamma)$ is singular if and only if $\gamma A_{0}\left(\mathbf{u}_{r}\right)+\omega A_{d}\left(\mathbf{u}_{r}\right)$ or $\gamma A_{0}\left(\mathbf{u}_{l}\right)-\omega A_{d}\left(\mathbf{u}_{l}\right)$ are singular. As a consequence, $\omega$ satisfies the dispersion equation if and only if there exists an integer $k \in\{1, \ldots, q\}$ such that

$$
\lambda_{k}\left(\mathbf{u}_{r}, e_{d}\right) \omega=-\gamma \quad \text { or } \quad \lambda_{k}\left(\mathbf{u}_{l}, e_{d}\right) \omega=\gamma,
$$

where $e_{d}:=(0, \ldots, 0,1) \in \mathbb{R}^{d}$. Assumption 3 shows that such values of $\omega$ are negative for $k=p+1, \ldots, q$ in the first case and $k=1, \ldots, p-1$ in the second case. Taking multiplicities into account, this shows that $\mathcal{E}^{-}(\mathbf{u}, 0, \gamma)$ (and therefore $\mathcal{E}^{-}(\mathbf{u}, \eta, \gamma)$ ) has dimension $N-1$ as long as $\gamma>0$.

For fixed $\underline{\eta} \neq 0$, the stable subspace $\mathcal{E}^{-}(\mathbf{u}, \eta, \gamma)$ admits a continuous extension when $(\eta, \gamma) \rightarrow(\underline{\eta}, 0)$, see [8] (the argument makes use of the compactness of Grassmanian manifolds); we still denote this extension by $\mathcal{E}^{-}(\mathbf{u}, \underline{\eta}, 0)$. Note that for $\gamma=0$, vectors in the extended stable subspace are not always boundary values of square integrable functions because of the possible occurrence of purely imaginary eigenmodes. This is widely detailed in $[5,12]$ for Euler equations of isentropic gas dynamics. 
We define the hemisphere of $\mathbb{R}^{d+1}$ as

$$
\Sigma_{+}:=\left\{(\eta, \gamma) \in \mathbb{R}^{d+1} \text { s.t. } \gamma^{2}+|\eta|^{2}=1 \text { and } \gamma \geqslant 0\right\} .
$$

The boundary of $\Sigma_{+}$will be denoted by $\Sigma_{0}$, that is,

$$
\Sigma_{0}:=\left\{(\eta, 0) \in \mathbb{R}^{d+1} \text { s.t. }|\eta|^{2}=1\right\} .
$$

Recall the following definition:

Definition 1 (Majda [24]). The planar shock $\mathbf{u}$ is said to satisfy the uniform stability condition if there exists a positive constant $c>0$ such that for all $(\eta, \gamma) \in \Sigma_{+}$, one has

$$
\forall(\chi, Z) \in \mathbb{C} \times \mathcal{E}^{-}(\mathbf{u}, \eta, \gamma), \quad|\chi b(\mathbf{u}, \eta, \gamma)+M(\mathbf{u}) Z| \geqslant c(|\chi|+|Z|) .
$$

By compactness of $\Sigma_{+}$, the uniform stability condition is equivalent to the requirement that for all $(\eta, \gamma) \in \Sigma_{+}$, the "critical" subspace

$$
\left\{(\chi, Z) \in \mathbb{C} \times \mathcal{E}^{-}(\mathbf{u}, \eta, \gamma) \text { s.t. } \chi b(\mathbf{u}, \eta, \gamma)+M(\mathbf{u}) Z=0\right\}
$$

is trivial, that is, reduced to $\{0\}$. The word "critical" is not standard but its use here intends to show the major place that is occupied by this subspace in the normal modes analysis.

Recall that a planar shock is uniformly stable if and only if solutions of (9) satisfy a maximal $L^{2}$ estimate as in the study of linear hyperbolic boundary value problems, see [24, Proposition 1]. The uniform stability condition is thus the direct extension of Kreiss' uniform condition for standard hyperbolic boundary value problems [21].

As noted by Majda [24, Lemma 4.1], the uniform stability condition enables to isolate the shock front $\Psi$ appearing in (11) in a single equation. We emphasize that this operation cannot be achieved for scalar conservation laws in space dimension more than 1.

Our approach is slightly different: we shall allow some instability but these instabilities can only stem from the traces of solutions to the dynamical system (12) and not from the shock front symbol $b$ defined by (13). More precisely, we make the following assumption:

Assumption 4. There exists a positive constant $c$ such that

$$
\forall(\eta, \gamma) \in \Sigma_{+}, \quad|b(\mathbf{u}, \eta, \gamma)| \geqslant c .
$$

Of course, an analogue estimate is valid for all states $u_{r}, u_{l}$ close to $\mathbf{u}_{r}, \mathbf{u}_{l}$.

Remark. Assumption 4 is equivalent (see [18, Chapter 4] or [20]) to the requirement that there exists a $\mathcal{C}^{\infty}$ mapping $P: \Sigma_{+} \rightarrow G l_{N}(\mathbb{C})$ such that

$$
\forall(\eta, \gamma) \in \Sigma_{+}, \quad P(\mathbf{u}, \eta, \gamma) b(\mathbf{u}, \eta, \gamma)=\left(\begin{array}{l}
\mathbf{0} \\
1
\end{array}\right),
$$

and one can even choose $P$ depending smoothly on the states $\mathbf{u}_{r}, \mathbf{u}_{l}$.

It is clear that (14) holds if $\mathbf{u}$ satisfies Majda's uniform stability condition. In a pseudodifferential setting, $b$ is an overdetermined elliptic symbol ( $\gamma$ is seen as a parameter and we are thus dealing with pseudodifferential symbols with a parameter). It is shown in [12] that Assumption 4 is met in some cases where the uniform stability condition is violated: the main example concerns shock waves in isentropic gas dynamics when the pressure law is not a convex function of the density. 
Assumption 4 enables us to reformulate boundary conditions (11) by isolating the unknown shock front $\Psi$ in a single equation. Because of (14), we can define for all $(\eta, \gamma) \in \mathbb{R}^{d} \times \mathbb{R}^{+} \backslash\{0\}$ the orthogonal projector

$$
\Pi(\mathbf{u}, \eta, \gamma) h:=h-\frac{\langle h, b(\mathbf{u}, \eta, \gamma)\rangle}{|b(\mathbf{u}, \eta, \gamma)|^{2}} b(\mathbf{u}, \eta, \gamma)
$$

Multiplying (11) by $\Pi$ and $b^{*}$ yields the new boundary conditions

$$
\begin{aligned}
& \Pi(\mathbf{u}, \eta, \gamma) M(\mathbf{u}) V(0)=\Pi(\mathbf{u}, \eta, \gamma) \widehat{G}, \\
& |b(\mathbf{u}, \eta, \gamma)|^{2} \Psi=\langle\widehat{G}-M(\mathbf{u}) V(0), b(\mathbf{u}, \eta, \gamma)\rangle .
\end{aligned}
$$

Observe that $b$ is homogeneous of degree 1 in $(\eta, \gamma)$ so (14) is equivalent to

$$
\forall(\eta, \gamma) \in \mathbb{R}^{d} \times \mathbb{R}^{+}, \quad c\left(\gamma^{2}+|\eta|^{2}\right) \leqslant|b(\mathbf{u}, \eta, \gamma)|^{2} .
$$

Using Schwarz' inequality in (15b), we obtain

$$
\forall(\eta, \gamma) \in \mathbb{R}^{d} \times \mathbb{R}^{+}, \quad\left(\gamma^{2}+|\eta|^{2}\right)|\Psi|^{2} \leqslant C\left(|\widehat{G}|^{2}+|V(0)|^{2}\right)
$$

for a suitable constant $C$ depending only on the shock $\mathbf{u}$. It is thus sufficient to get an estimate on $V(0)$ and we shall get from (16) an estimate on $\Psi$. In order to obtain the desired estimate on $V(0)$, we attack the following boundary value problem:

$$
\left\{\begin{array}{l}
\frac{\mathrm{d} V}{\mathrm{~d} x_{d}}=\mathcal{A}(\mathbf{u}, \eta, \gamma) V+\mathcal{A}_{d}(\mathbf{u})^{-1} \widehat{F}, \quad \text { for } x_{d}>0, \\
\Pi(\mathbf{u}, \eta, \gamma) M(\mathbf{u}) V(0)=\Pi(\mathbf{u}, \eta, \gamma) \widehat{G} .
\end{array}\right.
$$

To avoid overloading the paper, we introduce the notation:

$$
\beta(\mathbf{u}, \eta, \gamma):=\Pi(\mathbf{u}, \eta, \gamma) M(\mathbf{u}) .
$$

This is the symbol of the "reduced" boundary conditions.

Isolating the front yields a boundary value problem where the boundary operator takes the form of a Fourier multiplier of order 0 . Indeed, the homogeneity property of $b$ implies that $\Pi$ is homogeneous of degree 0 with respect to $(\eta, \gamma)$. More precisely, $\Pi$ is a pseudodifferential symbol of degree 0 ( $\gamma$ is seen as a parameter), and so is also $\beta$. We refer to [11] for a detailed study of pseudodifferential calculus with a parameter; the introduction of a parameter in Bony's paradifferential calculus [10,28] has been achieved in [29], see also [27].

Our final assumption is that the boundary conditions defined by the symbol $\beta$ satisfy the Kreiss-Lopatinskii condition but violate the uniform Kreiss-Lopatinskii condition. It is important for what follows that this assumption is met by all planar shock waves close to $\mathbf{u}$ :

Assumption 5. For all planar shock waves $u$ close to the reference shock wave $\mathbf{u}$, the following properties hold:

- If $(\eta, \gamma) \in \Sigma_{+}$and $\gamma>0$, the reduced critical subspace

$$
\left\{Z \in \mathcal{E}^{-}(u, \eta, \gamma) \text { s.t. } \beta(u, \eta, \gamma) Z=0\right\}
$$

is trivial.

- If $(\underline{\eta}, 0) \in \Sigma_{0}$ is a point where the reduced critical subspace

$$
\left\{Z \in \mathcal{E}^{-}(u, \underline{\eta}, 0) \text { s.t. } \beta(u, \underline{\eta}, 0) Z=0\right\}
$$

is nontrivial, then there exists a neighborhood $\mathcal{V}$ of $(\underline{\eta}, 0)$ in $\Sigma_{+}$and a constant $c>0$ such that

$$
\forall(\eta, \gamma) \in \mathcal{V}, \forall Z \in \mathcal{E}^{-}(u, \eta, \gamma), \quad|\beta(u, \eta, \gamma) Z| \geqslant c \gamma|Z| .
$$


Furthermore, there exists a $\mathcal{C}^{\infty}$ mapping $Q(u, \cdot): \mathcal{V} \rightarrow G l_{2 N}(\mathbb{C})$ depending smoothly on $u$ such that, for all $z=(\eta, \gamma) \in \mathcal{V}$, one has

$$
Q(u, z) \mathcal{A}(u, z) Q(u, z)^{-1}=\left(\begin{array}{lll}
\omega_{1}(z) I_{n_{1}} & & \\
& \ddots & \\
& & \omega_{J}(z) I_{n_{J}}
\end{array}\right),
$$

where the $\omega_{j}$ 's are complex valued functions, and $I_{n_{j}}$ is the identity matrix with size $n_{j}$. Moreover, the integers $n_{j}$ 's are independant of $(u, z)$, and the $\omega_{j}$ 's satisfy

$$
\operatorname{Re} \omega_{j}(z) \geqslant c \gamma
$$

or

$$
\operatorname{Re} \omega_{j}(z) \leqslant-c \gamma,
$$

for all $z \in \mathcal{V}$. In applications, it may happen that some of the $\omega_{j}$ 's have negative or positive real part when $\gamma=0$. In such a case, one would have

$$
\operatorname{Re} \omega_{j}(z) \geqslant c \quad(\text { or } \leqslant-c)
$$

for all $z \in \mathcal{V}$.

The terminology we shall use throughout the paper is the following:

Definition 2. The planar shock wave $\mathbf{u}$ is said to be weakly stable if it satisfies Assumptions 3-5. In particular, all planar shock waves close to $\mathbf{u}$ are also weakly stable.

Before stating our main result on such weakly stable planar shocks, let us explain in a few words the meaning of Assumption 5. The first condition asserts that the shock wave is not violently unstable: it must satisfy the analogue of the Kreiss-Lopatinskii condition for linear hyperbolic boundary value problems. The second condition asserts that the uniform stability condition is violated "at order 1". Recall that when Majda's uniform stability condition is met, one has an estimate of the type

$$
\forall(\eta, \gamma) \in \mathcal{V}, \forall Z \in \mathcal{E}^{-}(u, \eta, \gamma), \quad|\beta(u, \eta, \gamma) Z| \geqslant c|Z| .
$$

In our study, the allowed instability yields a power of $\gamma$ in (18).

When $(\eta, \gamma)$ tends to an instability point $(\eta, 0)$, part of the stable and unstable subspaces of $\mathcal{A}(u, \eta, \gamma)$ form a central subspace. The last requirement of Assumption 5 implies that the restriction of $\mathcal{A}(u, \eta, \gamma)$ to this subspace (that is central when $\gamma=0$ ) is smoothly diagonalizable. In gas dynamics, there are examples of shock waves for which the uniform stability condition breaks down at a point where the symbol is not smoothly diagonalizable, see $[12,24]$. Unfortunately, we have not been able to deal with this case: one major problem is the failure of the differentiability of the eigenmodes at such points. We refer to the work by Okhubo and Shirota [30] for some aspects of these phenomena.

Remark. Making Assumption 5 for all planar shock waves close to $\mathbf{u}$ is not very restrictive (first because it is satisfied in both examples we study). In fact, if Assumption 5 is satisfied by the shock wave $\mathbf{u}$, then it is automatically satisfied by all shock waves close to u provided that all the $\omega_{j}$ 's are purely imaginary when $\gamma=0$ in the neighborhood of the instability points, see [7]. This theoretical result applies to weakly stable shock waves in isentropic gas dynamics, see [12]. In the case of phase transitions in a van der Waals fluid, instability points belong to a region of the parameters $(\eta, \gamma)$ where some eigenmodes have negative real part and it is not a direct consequence of [7] that Assumption 5 is satisfied by all planar phase transitions close to the reference phase transition $\mathbf{u}$ (but fortunately the calculations show that it is true). 


\subsection{The weak stability of planar shock waves}

In this paragraph, we show that if $\mathbf{u}$ is a weakly stable planar shock, then the constant coefficients boundary value problem (9) is "well-posed", in the sense that solutions to (9) satisfy an a priori energy estimate. Since we deal with shock waves that violate Majda's uniform stability condition, the energy estimate will involve losses of derivatives with respect to the source terms $f$ and $g$.

We need first of all to introduce a few notations. Denote by $\Omega$ and $\omega$ the domains

$$
\Omega:=\mathbb{R}_{+}^{d+1}=\left\{\left(x_{0}, \ldots, x_{d}\right) \in \mathbb{R}^{d+1} \text { s.t. } x_{d}>0\right\} \quad \text { and } \omega:=\mathbb{R}^{d}=\partial \Omega .
$$

For $\gamma>0$ and $s \in \mathbb{R}$ we define the following symbols

$$
\forall \xi \in \mathbb{R}^{d}, \quad \lambda^{s, \gamma}(\xi):=\left(\gamma^{2}+|\xi|^{2}\right)^{s / 2}
$$

The usual Sobolev spaces $H^{s}(\omega)$ are equipped with the following weighted norms (depending on the positive parameter $\gamma$ ):

$$
\|v\|_{s, \gamma}^{2}:=\frac{1}{(2 \pi)^{d}} \int_{\mathbb{R}^{d}} \lambda^{2 s, \gamma}(\xi)|\hat{v}(\xi)|^{2} \mathrm{~d} \xi .
$$

We shall write $\|\cdot\|_{0}$ instead of $\|\cdot\|_{0, \gamma}$ since there is no dependance on the parameter $\gamma$ for the $L^{2}$ norm. These weighted norms enable to construct a parameter version of the classical pseudodifferential calculus which is of constant use in the study of hyperbolic boundary value problems, see, e.g., [21,24,27].

The space $L^{2}\left(\mathbb{R}^{+}, H^{s}\left(\mathbb{R}^{d}\right)\right)$ is also equipped with the weighted norm:

$$
\|v\|_{s, \gamma}^{2}:=\int_{0}^{+\infty}\left\|v\left(\cdot, x_{d}\right)\right\|_{s, \gamma}^{2} \mathrm{~d} x_{d} .
$$

We shall also write $\|\cdot\|_{0}$ instead of $\|\cdot\|_{0, \gamma}$. Typically, we shall use the spaces $L^{2}(\Omega)$ and $L^{2}\left(\mathbb{R}^{+}, H^{1}\left(\mathbb{R}^{d}\right)\right)$.

Eventually, the scalar products of $L^{2}(\omega)$ and $L^{2}(\Omega)$ will be denoted as follows:

$$
\langle f, g\rangle_{L^{2}(\omega)}:=\int_{\omega} f(y) \overline{g(y)} \mathrm{d} y, \quad\langle\langle f, g\rangle\rangle_{L^{2}(\Omega)}:=\int_{\Omega} f(x) \overline{g(x)} \mathrm{d} x .
$$

The Laplace transform performed in the normal modes analysis amounts to working with the new functions $\tilde{v}:=\exp (-\gamma t) v$ and $\tilde{\psi}:=\exp (-\gamma t) \psi$, with $\gamma>0$. This leads to the introduction of the "weighted" operators:

$$
\mathcal{L}_{\mathbf{u}}^{\gamma} \tilde{v}:=\mathcal{L}_{\mathbf{u}} \tilde{v}+\gamma \mathcal{A}_{0}(\mathbf{u}) \tilde{v} \quad \text { and } \quad \mathcal{B}_{\mathbf{u}}^{\gamma}(\tilde{v}, \tilde{\psi}):=\mathcal{B}_{\mathbf{u}}(\tilde{v}, \tilde{\psi})+\gamma \tilde{\psi} b_{0}(\mathbf{u})
$$

One easily checks that $(9)$ is equivalent to

$$
\begin{cases}\mathcal{L}_{\mathbf{u}}^{\gamma} \tilde{v}=\exp (-\gamma t) f, & \text { for } x_{d}>0 \\ \mathcal{B}_{\mathbf{u}}^{\gamma}(\tilde{v}, \tilde{\psi})=\exp (-\gamma t) g, & \text { for } x_{d}=0\end{cases}
$$

For convenience, we drop the tilda from $\tilde{v}$ and $\tilde{\psi}$ (keeping in mind that all functions have been multiplied by a decreasing exponential function and therefore also depend on the parameter $\gamma$ ). Our result on weakly stable planar shock waves can be stated as follows:

Theorem 2.1. Let $\mathbf{u}$ be a weakly stable planar shock. Then for all $v \in H^{2}(\Omega)$, for all $\psi \in H^{2}(\omega)$ and for all $\gamma \geqslant 1$, one has:

$$
\gamma\|v\|_{0}^{2}+\left\|v_{\left.\right|_{x_{d}}=0}\right\|_{0}^{2}+\|\psi\|_{1, \gamma}^{2} \lesssim \frac{1}{\gamma^{3}}\left\|\mathcal{L}_{\mathbf{u}}^{\gamma}(v, \psi)\right\|_{1, \gamma}^{2}+\frac{1}{\gamma^{2}}\left\|\mathcal{B}_{\mathbf{u}}^{\gamma}(v, \psi)\right\|_{1, \gamma}^{2} .
$$


Energy estimates in Sobolev spaces of higher order are available, provided that $v$ and $\psi$ are sufficiently regular. Similar estimates also hold for planar shock waves u close to $\mathbf{u}$.

\subsection{Proof of Theorem 2.1}

The proof of Theorem 2.1 follows the earlier works of Kreiss [21] and Majda [24] with suitable modifications. It can be found in [12] in the case of isentropic gas dynamics.

Recall first that Assumption 2 ensures that system (1) satisfies the so-called block structure condition. More precisely, we have:

Proposition 2.1 (Block structure condition [26]). If Assumption 2 is satisfied, then for all $u$ close to $\mathbf{u}$, for all $\underline{z} \in \Sigma_{+}$, there exists a neighborhood $\mathcal{V}$ of $\underline{z}$ in $\Sigma_{+}$and regular matrices $Q(u, z)$ depending smoothly on $u$ and $z \in \mathcal{V}$ such that

$$
\forall z \in \mathcal{V}, \quad Q(u, z) \mathcal{A}(u, z) Q(u, z)^{-1}=\left(\begin{array}{ccccc}
a^{-}(z) & & & & \\
& a^{+}(z) & & \mathbf{0} & \\
& & a_{1}(z) & & \\
& \mathbf{0} & & \ddots & \\
& & & & a_{K}(z)
\end{array}\right) .
$$

Furthermore, the spectrum of $a^{-}(z)$ (respectively $a^{+}(z)$ ) is contained in the half-plane $\{\operatorname{Re} \zeta<0\}$ (respectively $\{\operatorname{Re} \zeta>0\})$, and for all $k=1, \ldots, K$, the matrix $a_{k}(z)$ has purely imaginary coefficients when $\gamma=0$ and $a_{k}(\underline{z})$ satisfies

$$
a_{k}(\underline{z})=\omega_{k} I+\mathrm{i} N_{k} \quad \text { with } \omega_{k} \in \mathrm{i} \mathbb{R} \text { and } N_{k}=\left(\begin{array}{cccc}
0 & 1 & 0 & 0 \\
0 & 0 & \ddots & 0 \\
\vdots & \ddots & 0 & 1 \\
0 & \ldots & 0 & 0
\end{array}\right) .
$$

Finally, the lower left-hand corner coefficient of $\partial a_{k} / \partial \gamma(\underline{z})$ is a nonzero real number. Note that the dimensions of the blocks $a^{ \pm}$and $a_{k}$ may depend on $\underline{z}$ but not on $z \in \mathcal{V}$.

Let us go back to Assumption 5: if $\underline{z}$ is a point of $\Sigma_{0}$ such that the critical subspace

$$
\left\{Z \in \mathcal{E}^{-}(u, \underline{z}) \text { s.t. } \beta(u, \underline{z}) Z=0\right\}
$$

is nontrivial, then we have assumed that all the blocks $a_{k}$ (defined by Proposition 2.1 above) have dimension 1 and that the blocks $a^{+}$and $a^{-}$can be chosen under diagonal form. In particular, when $z$ belongs to a suitable neighborhood of $\underline{z}, a_{k}(z)$ is a complex number $\omega_{k}$ such that $\omega_{k} \in \mathbb{i} \mathbb{R}$ when $\gamma=0$ and $\partial \omega_{k} / \partial \gamma(\underline{z})$ is real and does not vanish. If $\operatorname{Re} \omega_{k}<0$ when $\gamma>0$, we obtain

$$
\operatorname{Re} \omega_{k}(z) \leqslant-c \gamma,
$$

whereas we obtain

$$
\operatorname{Re} \omega_{k}(z) \geqslant c \gamma
$$

if $\operatorname{Re} \omega_{k}>0$ when $\gamma>0$. In both cases, $c$ is a positive constant depending on $u$ and $z$. We refer to [12] for a detailed study of the block structure condition in the case of isentropic gas dynamics. Assumption 5 then simply amounts to counting the multiplicities of the eigenvalues of $\mathcal{A}(u, z)$ near instability points.

We are now going to construct a microlocal symmetrizer in order to prove (20). 
- Let $\underline{z} \in \Sigma_{+} \backslash \Sigma_{0}$. Since $\mathcal{A}(u, \underline{z})$ has no purely imaginary eigenvalue (by Hersh's result [17]), there exists a neighborhood $\mathcal{V}$ of $z$ in $\Sigma_{+}$and invertible matrices $Q(u, z)$ depending smoothly on $z \in \mathcal{V}$ (and also smoothly on $u$ close to $\mathbf{u}$ ) such that

$$
\forall z \in \mathcal{V}, \quad Q(u, z) \mathcal{A}(u, z) Q(u, z)^{-1}=\left(\begin{array}{cc}
a^{-}(z) & \mathbf{0} \\
\mathbf{0} & a^{+}(z)
\end{array}\right),
$$

with

$$
\operatorname{Sp} a^{-}(\underline{z}) \subset\{\operatorname{Re} \zeta<0\} \quad \text { and } \quad \operatorname{Sp} a^{+}(\underline{z}) \subset\{\operatorname{Re} \zeta>0\} .
$$

Matrices $a^{-}(z)$ and $a^{+}(z)$ are defined by Dunford's formula, see [11,20]. As noted in [26], this reduction explains why the block structure condition needs only to be checked in the neighborhood of points belonging to $\Sigma_{0}$. By Lyapunov's Theorem, see [4], there exists two positive definite hermitian matrices $\mathrm{H}^{+}$and $\mathrm{H}^{-}$such that

$$
\operatorname{Re}\left(H^{ \pm} a^{ \pm}(\underline{z})\right)= \pm I \text {. }
$$

For convenience, we define the real part of a square matrix $N$ as $\left(N+N^{*}\right) / 2$ and we do the same for operators on a Hilbert space. Following Kreiss [21] (see also [11]), we choose $r$ of the form

$$
r(z):=\left(\begin{array}{cc}
-H^{-} & \mathbf{0} \\
\mathbf{0} & \alpha H^{+}
\end{array}\right),
$$

where $\alpha$ is a real number greater than 1 , to be chosen large enough. Because the critical subspace is trivial when $z$ belongs to a neighborhood of $\underline{z}$, it is proved in [11] that for $\alpha$ large enough, the following estimates hold

$$
\begin{aligned}
& \operatorname{Re}\left(r(z) Q(u, z) \mathcal{A}(u, z) Q(u, z)^{-1}\right) \geqslant \frac{1}{2} I, \\
& r(z)+C \tilde{\beta}(u, z)^{*} \tilde{\beta}(u, z) \geqslant c I, \quad \text { where } \tilde{\beta}(u, z):=\beta(u, z) Q(u, z)^{-1} .
\end{aligned}
$$

Constants $c$ and $C$ are positive and depend only on $(u, \underline{z})$.

- Let now $z \in \Sigma_{0}$ be a point where the critical subspace is trivial. It appears from Proposition 2.1 that Jordan blocks may occur in the reduction of the symbol $\mathcal{A}$. In such a case, the construction of the symmetrizer is rather technical. We refer to $[8,11,12,21,29,31]$ for the details. Following these anterior works, we conclude that there exists a neighborhood $\mathcal{V}$ of $\underline{z}$ in $\Sigma_{+}$and $\mathcal{C}^{\infty}$ matrix valued mappings $r$ and $Q(u, \cdot)$ defined on $\mathcal{V}$ such that for all $z \in \mathcal{V}, r(z)$ is hermitian, $Q(u, z)$ is regular (that is, invertible) and

$$
\begin{aligned}
& \operatorname{Re}\left(r(z) Q(u, z) \mathcal{A}(u, z) Q(u, z)^{-1}\right) \geqslant c \gamma I, \\
& r(z)+C \tilde{\beta}(u, z)^{*} \tilde{\beta}(u, z) \geqslant c I, \quad \tilde{\beta}(u, z):=\beta(u, z) Q(u, z)^{-1} .
\end{aligned}
$$

- Let $\underline{z} \in \Sigma_{0}$ be a point where the critical subspace is nontrivial. From Assumption 5, we know that there exists a neighborhood $\mathcal{V}$ of $\underline{z}$ in $\Sigma_{+}$and invertible matrices $Q(u, z)$, depending smoothly on $z \in \mathcal{V}$, such that

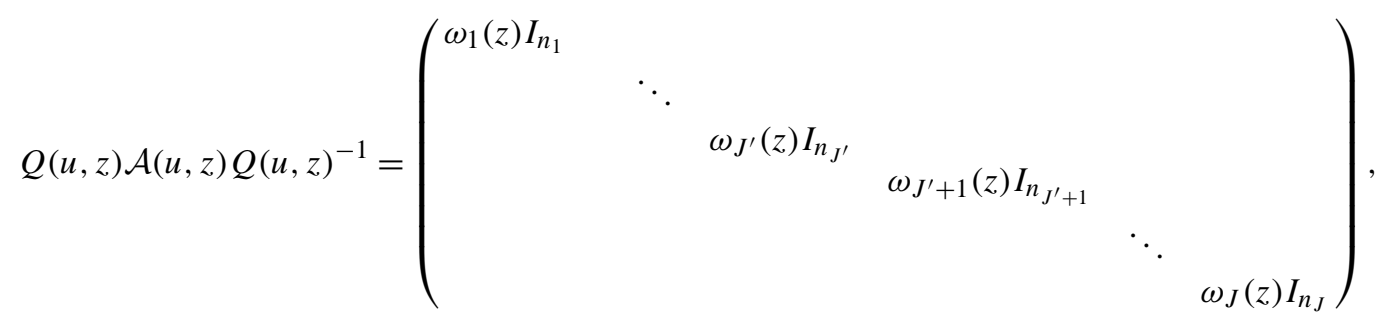

where the $\omega_{j}$ 's have negative real part when $\gamma>0$ and $j=1, \ldots, J^{\prime}$ and have positive real part when $\gamma>0$ and $j=J^{\prime}+1, \ldots, J$. We have just reordered the diagonal blocks appearing in Assumption 3. Let us remark that the 
stable subspace $\mathcal{E}^{-}(u, z)$ (that has dimension $N-1$ for all $z$ ) is spanned by the $N-1$ first column vectors of $Q(u, z)^{-1}$. In particular, we have

$$
n_{1}+\cdots+n_{J^{\prime}}=N-1 \text { and } n_{J^{\prime}+1}+\cdots+n_{J}=N+1 .
$$

For $z \in \mathcal{V}$, we choose $r(z)$ of the form

$$
r(z):=\left(\begin{array}{ll}
-\gamma^{2} I_{N-1} & \\
& \alpha I_{N+1}
\end{array}\right),
$$

where $\alpha$ is a real number greater than 1, to be fixed large enough. Because of the local behavior of the $\omega_{j}$ 's, see (21) and (22), we have

$$
\operatorname{Re}\left(r(z) Q(u, z) \mathcal{A}(u, z) Q(u, z)^{-1}\right) \geqslant c\left(\begin{array}{ll}
\gamma^{3} I_{N-1} & \\
& \alpha \gamma I_{N+1}
\end{array}\right),
$$

for all $z \in \mathcal{V}$. If $W \in \mathbb{C}^{2 N}$, we denote by $W^{-}$the vector composed by the $N-1$ first coordinates of $W$ and $W^{+}$the vector composed by the $N+1$ last coordinates of $W$ (this corresponds to a decomposition between the incoming part and the outgoing part), so we can write

$$
\beta(u, z) Q(u, z)^{-1} W=\beta(u, z) Q(u, z)^{-1}\left(\begin{array}{c}
W^{-} \\
0
\end{array}\right)+\beta(u, z) Q(u, z)^{-1}\left(\begin{array}{c}
0 \\
W^{+}
\end{array}\right),
$$

and using (18), we have

$$
\left|\beta(u, z) Q(u, z)^{-1}\left(\begin{array}{c}
W^{-} \\
0
\end{array}\right)\right| \geqslant c \gamma\left|W^{-}\right|,
$$

which implies

$$
\gamma^{2}\left|W^{-}\right|^{2} \lesssim\left|W^{+}\right|^{2}+|\tilde{\beta}(u, z) W|^{2}, \quad \tilde{\beta}(u, z):=\beta(u, z) Q(u, z)^{-1} .
$$

Choosing $\alpha$ large enough yields

$$
r(z)+C \tilde{\beta}(u, z)^{*} \tilde{\beta}(u, z) \geqslant c \gamma^{2} I,
$$

for all $z \in \mathcal{V}$. This completes the microlocal construction of the symmetrizer.

- We now turn to the proof of (20). For all $\underline{z} \in \Sigma_{+}$, the previous analysis establishes the existence of a neighborhood $\mathcal{V}$ of $\underline{z}$ in $\Sigma_{+}$and of smooth mappings $r$ and $Q$ with suitable properties. Because $\Sigma_{+}$is a smooth compact manifold, there exists a finite covering $\mathcal{V}_{i}, 1 \leqslant i \leqslant I$, of $\Sigma_{+}$by such neighborhoods and a smooth partition of unity $\chi_{i}, 1 \leqslant i \leqslant I$, subordinated to this covering. Functions $\chi_{i}$ are $\mathcal{C}^{\infty}$, nonnegative and satisfy

$$
\forall i=1, \ldots, I \quad \operatorname{Supp} \chi_{i} \subset \mathcal{V}_{i} \quad \text { and } \quad \sum_{i=1}^{I} \chi_{i}^{2} \equiv 1 .
$$

Let now $v \in H^{2}(\Omega)$ and $\psi \in H^{2}(\omega)$. We denote by $V\left(\eta, x_{d}\right)$ the Fourier transform of $v$ with respect to the $d$ first variables $\left(x_{0}, \ldots, x_{d-1}\right)$. We also define

$$
F:=\mathcal{L}_{\mathbf{u}}^{\gamma}(v, \psi) \in H^{1}(\Omega), \quad G:=\mathcal{B}_{\mathbf{u}}^{\gamma}(v, \psi) \in H^{1}(\omega)
$$

We extend all mappings $r_{i}$ and $Q_{i}$ on all $\Sigma_{+}$assuming them to be constant outside of $\mathcal{V}_{i}$ (this is of pure convenience since only the value of these mappings on the support of $\chi_{i}$ will be involved in the following calculations). Then we extend $\chi_{i}$ and $Q_{i}$ as homogeneous functions of degree 0 in $(\eta, \gamma)$. For $z=(\eta, \gamma) \in \mathbb{R}^{d} \times \mathbb{R}^{+}$, we define

$$
V_{i}\left(z, x_{d}\right):=\chi_{i}(z) Q_{i}(\mathbf{u}, z) V\left(\eta, x_{d}\right) .
$$

We thus get the relation

$$
\frac{\mathrm{d} V_{i}}{\mathrm{~d} x_{d}}=Q_{i}(\mathbf{u}, z) \mathcal{A}(\mathbf{u}, z) Q_{i}(\mathbf{u}, z)^{-1} V_{i}\left(z, x_{d}\right)+\chi_{i}(z) Q_{i}(\mathbf{u}, z) \mathcal{A}_{d}(\mathbf{u})^{-1} \widehat{F}\left(\eta, x_{d}\right) .
$$


If $\mathcal{V}_{i}$ is a neighborhood of a point $z_{i}$ where the critical subspace is trivial, we extend $r_{i}$ as a homogeneous function of degree 0 in $(\eta, \gamma)$. We take the scalar product of (23) with $r_{i}(z) V_{i}\left(z, x_{d}\right)$ and integrate with respect to $\left(\eta, x_{d}\right), \gamma$ being fixed. Using the inequalities

$$
\begin{aligned}
& \operatorname{Re}\left(r_{i}(z) Q_{i}(\mathbf{u}, z) \mathcal{A}(\mathbf{u}, z) Q_{i}(\mathbf{u}, z)^{-1}\right) \geqslant c \gamma I, \\
& r_{i}(z)+C \tilde{\beta}_{i}(\mathbf{u}, z)^{*} \tilde{\beta}_{i}(\mathbf{u}, z) \geqslant c I,
\end{aligned}
$$

we obtain Kreiss' maximal $L^{2}$ inequality

$$
\gamma\left\|\chi_{i} V\right\|_{0}^{2}+\left\|\chi_{i} V_{x_{d}=0}\right\|_{0}^{2} \lesssim \frac{1}{\gamma}\left\|\chi_{i} \widehat{F}\right\|_{0}^{2}+\left\|\chi_{i} \Pi \widehat{G}\right\|_{0}^{2} \lesssim \frac{1}{\gamma^{3}}\left\|\chi_{i} \lambda^{1, \gamma} \widehat{F}\right\|_{0}^{2}+\frac{1}{\gamma^{2}}\left\|\chi_{i} \lambda^{1, \gamma} \Pi \widehat{G}\right\|_{0}^{2} .
$$

If $\mathcal{V}_{i}$ is a neighborhood of a point $\underline{z_{i}}$ where the critical subspace is nontrivial, we extend $r_{i}$ as a homogeneous function of degree 2 in $(\eta, \gamma)$. We have

$$
r_{i}(z)+C \lambda^{2, \gamma}(\eta) \tilde{\beta}_{i}(\mathbf{u}, z)^{*} \tilde{\beta}_{i}(\mathbf{u}, z) \geqslant c \gamma^{2} I,
$$

and therefore, taking the scalar product of (23) with $r_{i}(z) V_{i}\left(z, x_{d}\right)$ and integrating with respect to $\left(\eta, x_{d}\right)$ yields the inequality

$$
-2 \operatorname{Re}\left\|r_{i} V_{i}, \chi_{i} Q_{i} \mathcal{A}_{d}^{-1} \widehat{F}\right\|_{L^{2}(\Omega)} \geqslant c \gamma^{2}\left\|\chi_{i} V_{\left.\right|_{d}=0}\right\|_{0}^{2}-C\left\|\chi_{i} \lambda^{1, \gamma} \Pi \widehat{G}\right\|_{0}^{2}+2 \operatorname{Re}\left\langle\left\langle V_{i}, r_{i} Q_{i} \mathcal{A} Q_{i}^{-1} V_{i} \|_{L^{2}(\Omega)} .\right.\right.
$$

Recall that $r_{i}$ has diagonal form

$$
r_{i}(z)=\left(\begin{array}{cc}
-\gamma^{2} I_{N-1} & \\
& \alpha \lambda^{2, \gamma}(\eta) I_{N+1}
\end{array}\right) \leqslant\left(\begin{array}{cc}
\gamma I_{N-1} & \\
& \sqrt{\alpha} \lambda^{1, \gamma}(\eta) I_{N+1}
\end{array}\right)^{2}=: s(z)^{2},
$$

and $r_{i}$ satisfies

$$
\operatorname{Re}\left(r_{i}(z) Q_{i}(z) \mathcal{A}(z) Q_{i}(z)^{-1}\right) \geqslant c \gamma s(z)^{2} .
$$

We have

$$
2 \operatorname{Re}\left\langle\left\|V_{i}, r_{i} Q_{i} \mathcal{A} Q_{i}^{-1} V_{i}\right\|_{L^{2}(\Omega)} \geqslant c \gamma\left\|s(z) V_{i}\right\|_{0}^{2},\right.
$$

and Young's inequality yields

$$
\begin{aligned}
-2 \operatorname{Re}\left\|r_{i} V_{i}, \chi_{i} Q_{i} \mathcal{A}_{d}^{-1} \widehat{F}\right\|_{L^{2}(\Omega)} & \leqslant \frac{c}{2} \gamma\left\|s(z) V_{i}\right\|_{0}^{2}+\frac{C}{\gamma}\left\|\chi_{i} s(z) \widehat{F}\right\|_{0}^{2} \\
& \leqslant \frac{c}{2} \gamma\left\|s(z) V_{i}\right\|_{0}^{2}+\frac{C}{\gamma}\left\|\chi_{i} \lambda^{1, \gamma} \widehat{F}\right\|_{0}^{2} .
\end{aligned}
$$

Eventually, we obtain

$$
\gamma^{3}\left\|\chi_{i} V\right\|_{0}^{2}+\gamma^{2}\left\|\chi_{i} V_{\left.\right|_{x_{d}}=0}\right\|_{0}^{2} \lesssim \frac{1}{\gamma}\left\|\chi_{i} \lambda^{1, \gamma} \widehat{F}\right\|_{0}^{2}+\left\|\chi_{i} \lambda^{1, \gamma} \Pi \widehat{G}\right\|_{0}^{2} .
$$

Since the $\chi_{i}$ 's form a partition of unity, Plancherel's Theorem yields

$$
\gamma\|v\|_{0}^{2}+\left\|v_{\left.\right|_{d}=0}\right\|_{0}^{2} \lesssim \frac{1}{\gamma^{3}}\left\|\mathcal{L}_{\mathbf{u}}^{\gamma} v\right\|_{1, \gamma}^{2}+\frac{1}{\gamma^{2}}\left\|\mathcal{B}_{\mathbf{u}}^{\gamma}(v, \psi)\right\|_{1, \gamma}^{2} .
$$

To conclude the proof, we integrate (16) with respect to $\eta \in \mathbb{R}^{d}$ :

$$
\|\psi\|_{1, \gamma}^{2} \lesssim\left\|v_{\left.\right|_{x_{d}=0}}\right\|_{0}^{2}+\left\|\mathcal{B}_{\mathbf{u}}^{\gamma}(v, \psi)\right\|_{0}^{2} \lesssim\left\|v_{\left.\right|_{x_{d}}=0}\right\|_{0}^{2}+\frac{1}{\gamma^{2}}\left\|\mathcal{B}_{\mathbf{u}}^{\gamma}(v, \psi)\right\|_{1, \gamma}^{2},
$$

and this gives (20). 
Remark. In [24, Proposition 2], Majda stated an energy estimate similar to (20) for isentropic Euler equations. We point out that his result was obtained under the assumption $\mathcal{L}_{\mathbf{u}}^{\gamma} v \equiv 0$. Theorem 2.1 thus extends this earlier result and indicates that losses of derivatives occur both in the interior domain and on the boundary. This shows a major difference between our analysis and earlier works such as [30,32].

\section{Variable coefficients analysis: the $L^{2}$ estimate}

Let $\mathbf{u}$ be a weakly stable planar shock. Because of Assumption 3, there exists an open set $\mathcal{U}$ in $\mathbb{R}^{N} \times \mathbb{R}^{N} \times \mathbb{R} \times$ $\mathbb{R}^{d-1}$ containing the origin such that for all $\left(w_{r}, w_{l}, \sigma, v\right) \in \mathcal{U}$, we have

$$
\begin{aligned}
& \mathbf{u}_{r}+w_{r} \in U, \quad \mathbf{u}_{l}+w_{l} \in U, \\
& \lambda_{p-1}\left(\mathbf{u}_{l}+w_{l}, \xi\right)<\sigma<\lambda_{p}\left(\mathbf{u}_{l}+w_{l}, \xi\right) \quad \text { and } \quad \lambda_{p}\left(\mathbf{u}_{r}+w_{r}, \xi\right)<\sigma<\lambda_{p+1}\left(\mathbf{u}_{r}+w_{r}, \xi\right),
\end{aligned}
$$

where $\xi:=(-v, 1) \in \mathbb{R}^{d}$. In other words, $\mathcal{U}$ is an open set such that all planar shocks associated with elements of $\mathcal{U}$ are $p$-shocks. Shrinking $\mathcal{U}$ if necessary, all planar shock waves associated with elements of $\mathcal{U}$ are weakly stable. We fix a compact subset $\mathcal{K} \subset \mathcal{U}$ and consider mappings $\dot{u}_{r}, \dot{u}_{l}, \varphi$ such that $\varphi$ is defined on $\mathbb{R}^{d}, \dot{u}_{r}$ (resp. $\dot{u}_{l}$ ) is defined on $\left\{x_{d} \geqslant \varphi\left(x_{0}, \ldots, x_{d-1}\right)\right\}$ (resp. $\left.\left\{x_{d} \leqslant \varphi\left(x_{0}, \ldots, x_{d-1}\right)\right\}\right)$. Eventually, we assume that $\left(\dot{u}_{r}, \dot{u}_{l}, \nabla \varphi\right)$ takes its values in $\mathcal{K}$ and is compactly supported. We define a function

$$
\mathbf{a}:= \begin{cases}\mathbf{u}_{r}+\dot{u}_{r}(\mathbf{x}) & \text { if } x_{d}>\varphi\left(x_{0}, \ldots, x_{d-1}\right), \\ \mathbf{u}_{l}+\dot{u}_{l}(\mathbf{x}) & \text { if } x_{d}<\varphi\left(x_{0}, \ldots, x_{d-1}\right)\end{cases}
$$

and make the following assumption:

Assumption 6. For all point $\mathbf{x}=\left(x_{0}, \ldots, x_{d}\right)$ such that $x_{d}=\varphi\left(x_{0}, \ldots, x_{d-1}\right)$, the function

$$
\mathbf{a}_{\mathbf{x}}:= \begin{cases}\mathbf{u}_{r}+\dot{u}_{r}(\mathbf{x}) & \text { if } y_{d}>\nabla \varphi \cdot\left(y_{0}, \ldots, y_{d-1}\right), \\ \mathbf{u}_{l}+\dot{u}_{l}(\mathbf{x}) & \text { if } y_{d}<\nabla \varphi \cdot\left(y_{0}, \ldots, y_{d-1}\right),\end{cases}
$$

is a planar shock wave. An equivalent formulation is that the Rankine-Hugoniot jump conditions are satisfied at each point $\mathbf{x}=\left(x_{0}, \ldots, x_{d-1}, \varphi\left(x_{0}, \ldots, x_{d-1}\right)\right)$ :

$$
\sum_{j=0}^{d-1} \partial_{j} \varphi\left[f_{j}(\mathbf{a})\right](\mathbf{x})=\left[f_{d}(\mathbf{a})\right](\mathbf{x}) .
$$

In the above relations, the gradient $\nabla \varphi$ is evaluated at $\left(x_{0}, \ldots, x_{d-1}\right)$.

The regularity of $\dot{u}_{r}, \dot{u}_{l}$ and $\varphi$ has not been precised. One can think of them as mild perturbations of the stationary shock wave $\mathbf{u}$ (some kind of first order correction in an asymptotic expansion). We shall be more precise in the sequel.

\subsection{The linearized equations}

We first straighten the variables to work in a fixed domain, as described in Section 1 . We still denote by a the function obtained after changing variables. We consider a family $u_{s}=\mathbf{a}+s v$ and $\varphi_{s}=\varphi+s \psi$. Then we define the linearized operators around $\mathbf{a}$ in the following way:

$$
\begin{aligned}
& \mathcal{L}_{\mathbf{a}}\left(v^{ \pm}, \psi\right):=\left.\frac{\mathrm{d}}{\mathrm{d} s} L\left(u_{s}^{ \pm}, \varphi_{s}\right) u_{s}^{ \pm}\right|_{s=0}, \\
& \mathcal{B}_{\mathbf{a}}(v, \psi):=\left.\frac{\mathrm{d}}{\mathrm{d} s} B\left(u_{s}^{+}, u_{s}^{-}, \varphi_{s}\right)\right|_{s=0} .
\end{aligned}
$$


A direct computation shows that

$$
\begin{aligned}
\mathcal{L}_{\mathbf{a}}\left(v^{ \pm}, \psi\right)= & \sum_{j=0}^{d-1} A_{j}\left(\mathbf{a}^{ \pm}\right) \partial_{j} v^{ \pm}+\sum_{j=0}^{d-1}\left[\nabla A_{j}\left(\mathbf{a}^{ \pm}\right) \cdot v^{ \pm}\right] \partial_{j} \dot{u}_{r, l}+\widetilde{A_{d}}\left(\mathbf{a}^{ \pm}, \nabla \varphi\right) \partial_{d} v^{ \pm} \\
& -\sum_{j=0}^{d-1} \partial_{j} \psi A_{j}\left(\mathbf{a}^{ \pm}\right) \partial_{d} \dot{u}_{r, l}+\left[\nabla_{u} \widetilde{A_{d}}\left(\mathbf{a}^{ \pm}, \nabla \varphi\right) \cdot v^{ \pm}\right] \partial_{d} \dot{u}_{r, l}, \quad \pm x_{d}>0,
\end{aligned}
$$

and

$$
\mathcal{B}_{\mathbf{a}}(v, \psi)=\sum_{j=0}^{d-1} \partial_{j} \psi\left[f_{j}(\mathbf{a})\right]-\widetilde{A_{d}}\left(\mathbf{a}^{+}, \nabla \varphi\right) v^{+}+\widetilde{A_{d}}\left(\mathbf{a}^{-}, \nabla \varphi\right) v^{-}, \quad x_{d}=0 .
$$

We decompose the linearized operator $\mathcal{L}_{\mathbf{a}}$ as

$$
\mathcal{L}_{\mathbf{a}}\left(v^{ \pm}, \psi\right)=L\left(\mathbf{a}^{ \pm}, \varphi\right) v^{ \pm}-\sum_{j=0}^{d-1} \partial_{j} \psi A_{j}\left(\mathbf{a}^{ \pm}\right) \partial_{d} \dot{u}_{r, l}-\mathcal{C}\left(\mathbf{a}^{ \pm}, \varphi\right) v^{ \pm},
$$

where $L$ is defined by (5a), (5b) and

$$
\mathcal{C}\left(\mathbf{a}^{ \pm}, \varphi\right) v^{ \pm}:=-\sum_{j=0}^{d-1}\left[\nabla A_{j}\left(\mathbf{a}^{ \pm}\right) \cdot v^{ \pm}\right] \partial_{j} \dot{u}_{r, l}-\left[\nabla_{u} \widetilde{A_{d}}\left(\mathbf{a}^{ \pm}, \nabla \varphi\right) \cdot v^{ \pm}\right] \partial_{d} \dot{u}_{r, l}
$$

is the zero order part (in $v^{ \pm}$) of $\mathcal{L}_{\mathbf{a}}$.

As was done in Section 2, we write the linearized equations as a first order system in $v:=\left(v^{+}, v^{-}\right)$and $\psi$ in the domain $\left\{x_{d}>0\right\}$. Define

$$
\begin{aligned}
& \mathcal{A}_{j}(\mathbf{a}):=\left(\begin{array}{cc}
A_{j}\left(\mathbf{a}^{+}\right) & \mathbf{0} \\
\mathbf{0} & A_{j}\left(\mathbf{a}^{-}\right)
\end{array}\right) \quad \text { for } 0 \leqslant j \leqslant d-1, \\
& \mathcal{A}_{d}(\mathbf{a}):=\left(\begin{array}{cc}
\widetilde{A_{d}}\left(\mathbf{a}^{+}, \nabla \varphi\right) & \mathbf{0} \\
\mathbf{0} & -\widetilde{A_{d}}\left(\mathbf{a}^{-}, \nabla \varphi\right)
\end{array}\right), \\
& b_{j}(\mathbf{a}):=\left[f_{j}(\mathbf{a})\right] \text { for } 0 \leqslant j \leqslant d-1 \quad \text { and } \quad M(\mathbf{a}):=\left(-\widetilde{A_{d}}\left(\mathbf{a}^{+}, \nabla \varphi\right) \quad \widetilde{A_{d}}\left(\mathbf{a}^{-}, \nabla \varphi\right)\right) .
\end{aligned}
$$

The linearized operators read

$$
\begin{aligned}
& \mathcal{L}_{\mathbf{a}}(v, \psi)=\sum_{j=0}^{d} \mathcal{A}_{j}(\mathbf{a}) \partial_{j} v-\sum_{j=0}^{d-1} \partial_{j} \psi \mathcal{A}_{j}(\mathbf{a}) \partial_{d} \dot{u}-\mathcal{C}(\mathbf{a}) v, \quad x_{d}>0, \\
& \mathcal{B}_{\mathbf{a}}(v, \psi)=\sum_{j=0}^{d-1} \partial_{j} \psi b_{j}(\mathbf{a})+M(\mathbf{a}) v, \quad x_{d}=0 .
\end{aligned}
$$

Recall that the perturbation $\left(\dot{u}_{r}, \dot{u}_{l}, \nabla \varphi\right)$ takes its values in the compact set $\mathcal{K} \subset \mathcal{U}$ so the matrix $\mathcal{A}_{d}(\mathbf{a})$ is regular.

We now introduce the positive weight $\gamma$, that is, we change functions $v$ and $\psi$ and deal with $\tilde{v}:=\exp (-\gamma t) v$ and $\tilde{\psi}:=\exp (-\gamma t) \psi$. As in Section 2, we introduce the weighted operators

$$
\mathcal{L}_{\mathbf{a}}^{\gamma}(\tilde{v}, \tilde{\psi}):=\mathcal{L}_{\mathbf{a}}(\tilde{v}, \tilde{\psi})+\gamma \mathcal{A}_{0}(\mathbf{a}) \tilde{v}-\gamma \tilde{\psi} \mathcal{A}_{0}(\mathbf{a}) \partial_{d} \dot{u} \quad \text { and } \quad \mathcal{B}_{\mathbf{a}}^{\gamma}(\tilde{v}, \tilde{\psi}):=\mathcal{B}_{\mathbf{a}}(\tilde{v}, \tilde{\psi})+\gamma \tilde{\psi} b_{0}(\mathbf{a})
$$

For simplicity, we drop the tildas.

We fix an integer $m>(d+5) / 2$ and assume that $\varphi \in H^{m+1 / 2}\left(\mathbb{R}^{d}\right)$ and $\dot{u}=\left(\dot{u}_{r}, \dot{u}_{l}\right) \in H^{m}(\Omega)$. Using some classical properties of Sobolev spaces, see [1], we have

$$
\nabla \varphi \in W^{2, \infty}\left(\mathbb{R}^{d}\right), \quad\left(\dot{u}_{r}, \dot{u}_{l}\right) \in W^{2, \infty}(\Omega) \quad \text { and } \quad\left(\dot{u}_{r}, \dot{u}_{l}\right) \in L^{2}\left(\mathbb{R}^{+}, W^{2, \infty}\left(\mathbb{R}^{d}\right)\right) .
$$


Because the coefficients of the linearized operators have limited smoothness, a convenient way to derive an energy estimate analogous to (20) is to use the paradifferential calculus of Bony, see [10,28]. With this strategy in mind, we are going to estimate the error between the linearized operators and their paralinearized version.

\subsection{The paralinearized equations}

We refer to Appendix for the definition of paradifferential symbols and for the main results of paradifferential calculus.

\subsubsection{Paralinearization of the boundary conditions}

Define the following symbols:

$$
\mathbf{b}\left(x_{0}, \ldots, x_{d-1}, \eta, \gamma\right):=\gamma \mathbf{b}_{0}\left(x_{0}, \ldots, x_{d-1}\right)+\mathrm{i} \sum_{j=0}^{d-1} \eta_{j} \mathbf{b}_{j}\left(x_{0}, \ldots, x_{d-1}\right),
$$

where

$$
\mathbf{b}_{j}\left(x_{0}, \ldots, x_{d-1}\right):=b_{j}\left(\mathbf{a}\left(x_{0}, \ldots, x_{d-1}, 0\right)\right) .
$$

Then we have $\mathbf{b}_{j} \in W^{2, \infty}\left(\mathbb{R}^{d}\right)$ and as a consequence $\mathbf{b} \in \Gamma_{2}^{1}$. Theorem A.5 yields

$$
\begin{aligned}
& \left\|\gamma \mathbf{b}_{0} \psi-T_{\gamma \mathbf{b}_{0}}^{\gamma} \psi\right\|_{1, \gamma} \lesssim\left\|\mathbf{b}_{0}\right\|_{W^{1, \infty}} \gamma\|\psi\|_{0} \lesssim\|\psi\|_{1, \gamma}, \\
& \left\|\mathbf{b}_{j} \partial_{j} \psi-T_{i \eta_{j} \mathbf{b}_{j}}^{\gamma} \psi\right\|_{1, \gamma}=\left\|\left(\mathbf{b}_{j}-T_{\mathbf{b}_{j}}^{\gamma}\right)\left(\partial_{j} \psi\right)\right\|_{1, \gamma} \lesssim\left\|\partial_{j} \psi\right\|_{0} \lesssim\|\psi\|_{1, \gamma},
\end{aligned}
$$

and we thus obtain

$$
\left\|\gamma \mathbf{b}_{0} \psi+\sum_{j=0}^{d-1} \partial_{j} \psi \mathbf{b}_{\mathbf{j}}-T_{\mathbf{b}}^{\gamma} \psi\right\|_{1, \gamma} \lesssim\|\psi\|_{1, \gamma} .
$$

We also define

$$
\mathbf{M}\left(x_{0}, \ldots, x_{d-1}\right):=M\left(\mathbf{a}\left(x_{0}, \ldots, x_{d-1}, 0\right)\right) \in W^{2, \infty}\left(\mathbb{R}^{d}\right),
$$

and Theorem A.5 yields

$$
\left\|\mathbf{M} v_{\left.\right|_{x_{d}=0}}-T_{\mathbf{M}}^{\gamma} v_{\left.\right|_{x_{d}=0}}\right\|_{1, \gamma} \lesssim\left\|v_{\left.\right|_{x_{d}}=0}\right\|_{0} .
$$

Combining (27) and (28), we get

$$
\left\|\mathcal{B}_{\mathbf{a}}^{\gamma}(v, \psi)-T_{\mathbf{b}}^{\gamma} \psi-T_{\mathbf{M}}^{\gamma} v_{\left.\right|_{x_{d}=0}}\right\|_{1, \gamma} \lesssim\|\psi\|_{1, \gamma}+\left\|v_{\left.\right|_{x_{d}=0}}\right\|_{0} .
$$

We shall therefore replace the linearized boundary operator $(v, \psi) \mapsto \mathcal{B}_{\mathbf{a}}^{\gamma}(v, \psi)$ by its paralinearized version $(v, \psi) \mapsto T_{\mathbf{b}}^{\gamma} \psi+T_{\mathbf{M}}^{\gamma} v$.

\subsubsection{Paralinearization of the evolution equations}

We are now going to paralinearize the evolution equations after multiplying by $\mathcal{A}_{d}^{-1}$. Define

$$
\mathbf{A}_{j}\left(x_{0}, \ldots, x_{d}\right):=\mathcal{A}_{j}\left(\mathbf{a}\left(x_{0}, \ldots, x_{d}\right)\right), \quad \mathbf{C}\left(x_{0}, \ldots, x_{d}\right):=\mathcal{C}\left(\mathbf{a}\left(x_{0}, \ldots, x_{d}\right)\right) .
$$

Because $\nabla \varphi \in W^{2, \infty}\left(\mathbb{R}^{d}\right)$ and $\mathbf{a} \in W^{2, \infty}(\Omega)$, we have $\mathbf{A}_{j} \in W^{2, \infty}(\Omega)$ and $\mathbf{C} \in W^{1, \infty}(\Omega)$. Recall that first order derivatives of $\dot{u}$ appear in the definition of $\mathbf{C}$, see (26), so we do not have $\mathbf{C} \in W^{2, \infty}(\Omega)$.

The matrix valued mapping $\mathbf{A}_{d}$ is uniformly invertible, namely

$$
\left\|\mathbf{A}_{d}^{-1}\right\|_{W^{2, \infty}(\Omega)} \leqslant C .
$$


Using the definition (19) and Theorem A.5, we obtain the following estimates

$$
\begin{aligned}
& \left\|\gamma \mathbf{A}_{d}^{-1} \mathbf{A}_{0} v-T_{\gamma \mathbf{A}_{d}^{-1} \mathbf{A}_{0}}^{\gamma} v\right\|_{1, \gamma} \lesssim\|v\|_{0}, \\
& \left\|\mathbf{A}_{d}^{-1} \mathbf{A}_{j} \partial_{j} v-T_{i \eta_{j} \mathbf{A}_{d}^{-1} \mathbf{A}_{j}}^{\gamma} v\right\|_{1, \gamma} \lesssim\|v\|_{0} \quad \text { for } 0 \leqslant j \leqslant d-1, \\
& \left\|\mathbf{A}_{d}^{-1} \mathbf{C} v-T_{\mathbf{A}_{d}^{-1} \mathbf{C}}^{\gamma} v\right\|_{1, \gamma} \lesssim\|v\|_{0} .
\end{aligned}
$$

Those estimates are obtained by a simple integration of the paraproduct estimates in $\mathbb{R}^{d}$ and from the definition of the paradifferential operators in a half-space, see appendix.

As for Eq. (12), for $\mathbf{x} \in \Omega, \eta \in \mathbb{R}^{d}$ and $\gamma \geqslant 0$, we define the symbol

$$
\mathbf{A}(\mathbf{x}, \eta, \gamma):=-\mathbf{A}_{d}(\mathbf{x})^{-1}\left(\gamma \mathbf{A}_{0}(\mathbf{x})+\mathrm{i} \sum_{j=0}^{d-1} \eta_{j} \mathbf{A}_{j}(\mathbf{x})\right) .
$$

It is clear that $\mathbf{A} \in \Gamma_{2}^{1}$ and the previous inequalities yield

$$
\left\|\gamma \mathbf{A}_{d}^{-1} \mathbf{A}_{0} v+\sum_{j=0}^{d-1} \mathbf{A}_{d}^{-1} \mathbf{A}_{j} \partial_{j} v-\mathbf{A}_{d}^{-1} \mathbf{C} v+T_{\mathbf{A}}^{\gamma} v+T_{\mathbf{A}_{d}^{-1}}^{\gamma} \mathbf{C} v\right\|_{1, \gamma} \lesssim\|v\|_{0} .
$$

We have thus estimated the error terms in $v$ when paralinearizing $\mathbf{A}_{d}^{-1} \mathcal{L}_{\mathbf{a}}^{\gamma}(v, \psi)$. We now turn to the error terms in $\psi$. There are two such terms that are

$$
\gamma \psi \mathbf{A}_{d}^{-1} \mathbf{A}_{0} \partial_{d} \dot{u}-T_{\gamma \mathbf{A}_{d}^{-1} \mathbf{A}_{0} \partial_{d} \dot{u}}^{\gamma} \psi \quad \text { and } \quad \mathbf{A}_{d}^{-1} \mathbf{A}_{j} \partial_{d} \dot{u} \partial_{j} \psi-T_{i \eta_{j} \mathbf{A}_{d}^{-1} \mathbf{A}_{j} \partial_{d} \dot{u}}^{\gamma} \psi, \quad 0 \leqslant j \leqslant d-1 .
$$

Using Theorem A.5 and the property $\partial_{d} \dot{u} \in L^{2}\left(\mathbb{R}^{+}, W^{1, \infty}\left(\mathbb{R}^{d}\right)\right)$, we obtain

$$
\begin{aligned}
& \left\|\gamma \psi \mathbf{A}_{d}^{-1} \mathbf{A}_{0} \partial_{d} \dot{u}-\gamma T_{\mathbf{A}_{d}^{-1} \mathbf{A}_{0} \partial_{d} \dot{u}}^{\gamma} \psi \mid\right\|_{1, \gamma} \lesssim \gamma\|\psi\|_{0} \lesssim\|\psi\|_{1, \gamma}, \\
& \left\|\mathbf{A}_{d}^{-1} \mathbf{A}_{j} \partial_{d} \dot{u} \partial_{j} \psi-T_{i \eta_{j} \mathbf{A}_{d}^{-1} \mathbf{A}_{j} \partial_{d} \dot{u}}^{\gamma} \psi\right\|_{1, \gamma} \lesssim\left\|\partial_{j} \psi\right\|_{0} \lesssim\|\psi\|_{1, \gamma} .
\end{aligned}
$$

We thus get the estimate

$$
\left\|\gamma \psi \mathbf{A}_{d}^{-1} \mathbf{A}_{0} \partial_{d} \dot{u}+\sum_{j=0}^{d-1} \mathbf{A}_{d}^{-1} \mathbf{A}_{j} \partial_{d} \dot{u} \partial_{j} \psi+T_{\mathbf{A} \partial_{d} \dot{u}}^{\gamma} \psi\right\|_{1, \gamma} \lesssim\|\psi\|_{1, \gamma} .
$$

Combining (30) and (31), we have proved

$$
\left\|\mathbf{A}_{d}^{-1} \mathcal{L}_{\mathbf{a}}^{\gamma}(v, \psi)-\partial_{d} v+T_{\mathbf{A}}^{\gamma} v+T_{\mathbf{A}_{d}^{-1} \mathbf{C}}^{\gamma} v-T_{\mathbf{A} \partial_{d} \dot{u}}^{\gamma} \psi\right\|_{1, \gamma} \lesssim\|v\|_{0}+\|\psi\|_{1, \gamma} .
$$

As for the boundary operator, we shall therefore replace the linearized operator $(v, \psi) \mapsto \mathbf{A}_{d}^{-1} \mathcal{L}_{\mathbf{a}}^{\gamma}(v, \psi)$ by its paralinearized version $(v, \psi) \mapsto \partial_{d} v-T_{\mathbf{A}}^{\gamma} v-T_{\mathbf{A}_{d}^{-1} \mathbf{C}}^{\gamma} v+T_{\mathbf{A}_{d} \dot{u}}^{\gamma} \psi$.

\subsubsection{Change of unknown function}

Unlike in the uniformly stable case, the linearized operator involves in our case a zero order operator in $v$ and a first order operator in $\psi$. We shall use a change of unknown functions, that is due to Alinhac, see [2], and that simplifies the expression of $\mathcal{L}_{\mathbf{a}}$. If we let $v=\dot{v}+\psi \partial_{d} \dot{u}$, we have

$$
\mathcal{L}_{\mathbf{a}}(v, \psi)=L(\mathbf{a}, \varphi) \dot{v}-\mathcal{C}(\mathbf{a}) \dot{v}+\psi \partial_{d}[L(\mathbf{a}, \varphi) \dot{u}] .
$$


The function $\dot{v}$ is referred to as the "good unknown" of the problem. We emphasize that this change of unknown functions yields an operator in $(\dot{v}, \psi)$ with only zero order term in $\psi$ : roughly speaking, the operator $\mathcal{L}_{\mathbf{a}}$ reduces to an operator with only first and zero order term in $\dot{v}$, since the zero order terms in $\psi$ will be easily estimated.

As regards the paralinearized equations, the previous result suggests to make the change of unknown functions $v:=\dot{v}+T_{\partial_{d} \dot{u}}^{\gamma} \psi$. We are going to show that the paralinearized operator is equal to $\partial_{d} \dot{v}-T_{\mathbf{A}}^{\gamma} \dot{v}-T_{\mathbf{A}_{d}^{-1} \mathbf{C}}^{\gamma} \dot{v}$ plus some error terms whose norm can be controlled.

A straightforward computation shows that

$$
\partial_{d} v-T_{\mathbf{A}}^{\gamma} v-T_{\mathbf{A}_{d}^{-1} \mathbf{C}}^{\gamma} v+T_{\mathbf{A} \partial_{d} \dot{u}}^{\gamma} \psi=\partial_{d} \dot{v}-T_{\mathbf{A}}^{\gamma} \dot{v}-T_{\mathbf{A}_{d}^{-1} \mathbf{C}}^{\gamma} \dot{v}+e_{1}-e_{2}+e_{3},
$$

where

$$
e_{1}:=T_{\partial_{d}^{2} \dot{u}}^{\gamma} \psi, \quad e_{2}:=T_{\mathbf{A}_{d}^{-1} \mathbf{C}}^{\gamma} T_{\partial_{d} \dot{u}}^{\gamma} \psi, \quad e_{3}:=\left(T_{\mathbf{A} \partial_{d} \dot{u}}^{\gamma}-T_{\mathbf{A}}^{\gamma} T_{\partial_{d} \dot{u}}^{\gamma}\right) \psi
$$

Because $\dot{u} \in H^{m}(\Omega)$ and $m>(d+5) / 2$, we have

$$
\left\|e_{i}\right\|_{1, \gamma} \lesssim\|\psi\|_{1, \gamma}, \quad 1 \leqslant i \leqslant 3,
$$

and therefore

$$
\left\|\left(\partial_{d} v-T_{\mathbf{A}}^{\gamma} v-T_{\mathbf{A}_{d}^{-1} \mathbf{C}}^{\gamma} v+T_{\mathbf{A} \partial_{d} \dot{u}}^{\gamma} \psi\right)-\left(\partial_{d} \dot{v}-T_{\mathbf{A}}^{\gamma} \dot{v}-T_{\mathbf{A}_{d}^{-1} \mathbf{C}}^{\gamma} \dot{v}\right)\right\|_{1, \gamma} \lesssim\|\psi\|_{1, \gamma} .
$$

As a consequence, we shall focus our attention on the operator $\partial_{d} \dot{v}-T_{\mathbf{A}}^{\gamma} \dot{v}-T_{\mathbf{A}_{d}^{-1}}^{\gamma} \mathbf{C}$ and try to derive an energy estimate for this operator.

Remark. After changing unknown functions, the paralinearized boundary operator reads $T_{\mathbf{b}}^{\gamma} \psi+T_{\mathbf{M}}^{\gamma} \dot{v}_{\left.\right|_{d}=0}+$ $T_{\mathbf{M}}^{\gamma} T_{\partial_{d} \dot{u}}^{\gamma} \psi$, and we see that the last term in this sum satisifies

$$
\left\|T_{\mathbf{M}}^{\gamma} T_{\partial_{d} \dot{u}}^{\gamma} \psi\right\|_{1, \gamma} \lesssim\|\psi\|_{1, \gamma} .
$$

To summarize, we have proved

$$
\begin{aligned}
& \left\|\mathbf{A}_{d}^{-1} \mathcal{L}_{\mathbf{a}}^{\gamma}(v, \psi)-\left(\partial_{d} \dot{v}-T_{\mathbf{A}}^{\gamma} \dot{v}-T_{\mathbf{A}_{d}^{-1} \mathbf{c}}^{\gamma} \dot{v}\right)\right\|_{1, \gamma} \leqslant C\left(\|v\|_{0}+\|\psi\|_{1, \gamma}\right), \\
& \left\|\mathcal{B}_{\mathbf{a}}^{\gamma}(v, \psi)-\left(T_{\mathbf{b}}^{\gamma} \psi+T_{\mathbf{M}}^{\gamma} \dot{v}_{\left.\right|_{d}=0}\right)\right\|_{1, \gamma} \leqslant C\left(\left\|v_{\left.\right|_{x_{d}=0}}\right\|_{0}+\|\psi\|_{1, \gamma}\right) .
\end{aligned}
$$

Furthermore, the relation $v=\dot{v}+T_{\partial_{d} \dot{u}}^{\gamma} \psi$ yields

$$
\begin{aligned}
& \left\|v_{\left.\right|_{d}=0}\right\|_{0}^{2} \leqslant 2\left(\left\|\dot{v}_{\left.\right|_{x_{d}=0}}\right\|_{0}^{2}+C\|\psi\|_{0}^{2}\right) \leqslant 2\left(\left\|\dot{v}_{\left.\right|_{d}=0}\right\|_{0}^{2}+\frac{C}{\gamma^{2}}\|\psi\|_{1, \gamma}^{2}\right), \\
& \|v\|_{0}^{2} \leqslant 2\left(\|\dot{v}\|_{0}^{2}+C\|\psi\|_{0}^{2}\right) \leqslant 2\left(\|\dot{v}\|_{0}^{2}+\frac{C}{\gamma^{2}}\|\psi\|_{1, \gamma}^{2}\right),
\end{aligned}
$$

and we thus get

$$
\gamma\|\dot{v}\|_{0}^{2}+\left\|\dot{v}_{\left.\right|_{d}=0}\right\|_{0}^{2} \geqslant \frac{1}{2}\left(\gamma\|v\|_{0}^{2}+\left\|v_{\left.\right|_{x_{d}=0}}\right\|_{0}^{2}\right)-\frac{C}{\gamma}\|\psi\|_{1, \gamma}^{2} .
$$

It is now clear that the change of unknown function is appropriate because an energy estimate of the same type as (20) for $(\dot{v}, \psi)$ will yield a similar energy estimate for $(v, \psi)$.

All constants appearing in (33) and (34) are uniform with respect to the norms of the perturbations and the compact set $\mathcal{K}$. Namely, if the perturbation $\left(\dot{u}_{r}, \dot{u}_{l}, \nabla \varphi\right)$ is valued in $\mathcal{K}$ and satisfies

$$
\|\varphi\|_{H^{m+1 / 2}(\omega)} \leqslant K, \quad\left\|\left(\dot{u}_{r}, \dot{u}_{l}\right)\right\|_{H^{m}(\Omega)} \leqslant K,
$$

for some constant $K>0$ and some integer $m>(d+5) / 2$, then the constants appearing in (33), (34) only depend on $K$ and $\mathcal{K}$. 


\subsubsection{Eliminating the shock front}

Using Assumption 4, we know that there exists a positive constant $c>0$ such that

$$
\mathbf{b}^{*}\left(x_{0}, \ldots, x_{d-1}, \eta, \gamma\right) \mathbf{b}\left(x_{0}, \ldots, x_{d-1}, \eta, \gamma\right) \geqslant c\left(\gamma^{2}+|\eta|^{2}\right) \text {. }
$$

The constant $c$ only depends on the compact set $\mathcal{K}$. The symbol $\mathbf{b}^{*} \mathbf{b}$ is of degree 2 and elliptic so, applying Gårding's inequality (Theorem A.3), we obtain

$$
\forall \gamma \geqslant \gamma_{0}, \quad\|\psi\|_{1, \gamma}^{2} \lesssim \operatorname{Re}\left\langle T_{\mathbf{b}^{*} \mathbf{b}}^{\gamma} \psi, \psi\right\rangle_{L^{2}} \lesssim\left\|T_{\mathbf{b}}^{\gamma} \psi\right\|_{0}^{2}+\operatorname{Re}\left\langle R^{\gamma} \psi, \psi\right\rangle_{L^{2}},
$$

where $\left\{R^{\gamma}\right\}$ is a family of order $\leqslant 1$. We thus get

$$
\|\psi\|_{1, \gamma}^{2} \lesssim\left\|T_{\mathbf{b}}^{\gamma} \psi\right\|_{0}^{2}+\|\psi\|_{1, \gamma}\|\psi\|_{0} \lesssim \frac{1}{\gamma}\|\psi\|_{1, \gamma}^{2}+\left\|T_{\mathbf{b}}^{\gamma} \psi\right\|_{0}^{2} .
$$

Up to a greater choice of $\gamma_{0}$, we finally get

$$
\begin{aligned}
\forall \gamma \geqslant \gamma_{0}, \quad\|\psi\|_{1, \gamma}^{2} & \lesssim\left\|T_{\mathbf{b}}^{\gamma} \psi\right\|_{0}^{2} \lesssim\left\|T_{\mathbf{b}}^{\gamma} \psi+T_{\mathbf{M}}^{\gamma} \dot{v}_{\left.\right|_{d}=0}\right\|_{0}^{2}+\left\|\dot{v}_{\left.\right|_{x_{d}}=0}\right\|_{0}^{2} \\
& \lesssim \frac{1}{\gamma^{2}}\left\|T_{\mathbf{b}}^{\gamma} \psi+T_{\mathbf{M}}^{\gamma} \dot{v}_{\left.\right|_{x_{d}}=0}\right\|_{1, \gamma}^{2}+\left\|\dot{v}_{\left.\right|_{x_{d}=0}}\right\|_{0}^{2},
\end{aligned}
$$

which is the variable coefficients version of (16).

Introducing the orthogonal projector $\Pi(y, \eta, \gamma)$ on $\mathbf{b}(y, \eta, \gamma)^{\perp}$, we have $\Pi \in \Gamma_{2}^{0}$ and $\Pi \mathbf{b} \equiv 0$. Theorem A.2 gives

$$
\left\|T_{\Pi}^{\gamma} T_{\mathbf{b}}^{\gamma} \psi\right\|_{1, \gamma} \lesssim\|\psi\|_{1, \gamma}, \quad\left\|T_{\Pi}^{\gamma} T_{\mathbf{M}}^{\gamma} \dot{v}_{\left.\right|_{x_{d}}=0}-T_{\Pi \mathbf{M}}^{\gamma} \dot{v}_{\left.\right|_{x_{d}}=0}\right\|_{1, \gamma} \lesssim\left\|\dot{v}_{\left.\right|_{x_{d}}=0}\right\|_{0} .
$$

Using the decomposition

$$
T_{\Pi \mathbf{M}}^{\gamma} \dot{v}_{\left.\right|_{x_{d}=0}}=\left(T_{\Pi \mathbf{M}}^{\gamma}-T_{\Pi}^{\gamma} T_{\mathbf{M}}^{\gamma}\right) \dot{v}_{\left.\right|_{x_{d}=0}}+T_{\Pi}^{\gamma}\left(T_{\mathbf{M}}^{\gamma} \dot{v}_{x_{d}=0}+T_{\mathbf{b}}^{\gamma} \psi\right)-T_{\Pi}^{\gamma} T_{\mathbf{b}}^{\gamma} \psi
$$

we end up with

$$
\left\|T_{\Pi \mathbf{M}}^{\gamma} \dot{v}_{\left.\right|_{x_{d}=0}}\right\|_{1, \gamma} \lesssim\left\|\dot{v}_{\left.\right|_{x_{d}=0}}\right\|_{0}+\left\|T_{\mathbf{b}}^{\gamma} \psi+T_{\mathbf{M}}^{\gamma} \dot{v}_{\left.\right|_{x_{d}}=0}\right\|_{1, \gamma}+\|\psi\|_{1, \gamma}
$$

From now on, we focus on the system

$$
\begin{cases}\partial_{d} \dot{v}-T_{\mathbf{A}}^{\gamma} \dot{v}-T_{\mathbf{A}_{d}^{-1} \mathbf{C}}^{\gamma} \dot{v}=F, & x_{d}>0, \\ T_{\mathbf{\Pi M}}^{\gamma} \dot{v}_{\left.\right|_{d}=0}=G, & x_{d}=0,\end{cases}
$$

and try to derive an energy estimate of the type

$$
\gamma\|\dot{v}\|_{0}^{2}+\left\|\dot{v}_{\left.\right|_{d}=0}\right\|_{0}^{2} \lesssim \frac{1}{\gamma^{3}}\|F\|_{1, \gamma}^{2}+\frac{1}{\gamma^{2}}\|G\|_{1, \gamma}^{2},
$$

for all $\gamma \geqslant \gamma_{0}$. Using (33)-(36) and a greater choice of the constant $\gamma_{0}$, we shall obtain the variable coefficients analogue of our basic estimate (20). A precise result will be stated in Section 3.6.

As was done in the constant coefficients case, we define the symbol $\boldsymbol{\beta}$ of the reduced boundary conditions:

$$
\forall(y, \eta, \gamma) \in \mathbb{R}^{d} \times \mathbb{R}^{d} \times \mathbb{R}^{+}, \quad \boldsymbol{\beta}(y, \eta, \gamma):=\Pi(y, \eta, \gamma) \mathbf{M}(y)
$$

Our goal is to derive an a priori estimate for the paralinearized system:

$$
\begin{cases}\partial_{d} \dot{v}-T_{\mathbf{A}}^{\gamma} \dot{v}-T_{\mathbf{A}_{d}^{-1} \mathbf{C}}^{\gamma} \dot{v}=F, & x_{d}>0, \\ T_{\beta}^{\gamma} \dot{v}_{\left.\right|_{d}=0}=G, & x_{d}=0 .\end{cases}
$$




\subsection{Geometrical assumptions}

Let us go back to Assumption 5. For all planar shock waves $u$ close to $\mathbf{u}$, we define the set of critical frequencies $\Sigma_{\mathrm{cr}}(u)$ as the set of those $(\eta, \gamma) \in \Sigma_{+}$such that the critical subspace

$$
\left\{Z \in \mathcal{E}^{-}(u, \eta, \gamma) \text { s.t. } \beta(u, \eta, \gamma) Z=0\right\}
$$

is not reduced to $\{0\}$. From Assumption 5, we know that frequencies $(\eta, \gamma) \in \Sigma_{\mathrm{cr}}(u)$ satisfy $\gamma=0$. Another requirement of Assumption 5 is that there exists a neighborhood $\mathcal{V}_{\mathrm{cr}}(u)$ of $\Sigma_{\mathrm{cr}}(u)$ in $\Sigma_{+}$and a smooth mapping $Q_{0}(u)$ defined on $\mathcal{V}_{\text {cr }}(u)$ with values in $G l_{2 N}(\mathbb{C})$ such that

$$
Q_{0} \mathcal{A} Q_{0}^{-1}=\left(\begin{array}{ccc}
\omega_{1} I_{n_{1}} & & \mathbf{0} \\
& \ddots & \\
\mathbf{0} & & \omega_{J} I_{n_{J}}
\end{array}\right)
$$

To deal with the variable coefficients case, we need to assume that $\Sigma_{\mathrm{cr}}(u)$ is endowed with an "equation". More precisely, we assume that there exists a smooth real valued function $\tilde{\sigma}$ (defined for all shock waves $u$ close to $\mathbf{u}$ ) such that

$$
\begin{aligned}
\Sigma_{\mathrm{cr}}(u) & =\left\{(\eta, \gamma) \in \Sigma_{+} \text {s.t. } \gamma+\mathrm{i} \tilde{\sigma}(u, \eta, \gamma)=0\right\} \\
& =\left\{(\eta, 0) \in \Sigma_{0} \text { s.t. } \tilde{\sigma}(u, \eta, 0)=0\right\} .
\end{aligned}
$$

We refer to Section 4 for an example. The function $\tilde{\sigma}$ is extended as an homogeneous mapping of degree 1 with respect to $(\eta, \gamma)$.

Recall that the perturbed planar shock $\mathbf{a}$ is assumed to satisfy the Rankine-Hugoniot relations at each point of the boundary $\partial \Omega$ (Assumption 6). We thus define the set of the so-called "critical points" as

$$
\Sigma_{\mathbf{c}}:=\left\{(x, \eta, \gamma) \in \partial \Omega \times \Sigma_{+} \text {s.t. }(\eta, \gamma) \in \Sigma_{\mathrm{cr}}(\mathbf{a}(x))\right\} .
$$

We already know from Assumption 5 that there exists a neighbourhood $\mathcal{V}_{\mathbf{c}}^{0}$ of $\Sigma_{\mathbf{c}}$ in $\partial \Omega \times \Sigma_{+}$such that the symbol $\mathbf{A}(z)$ is diagonalizable on $\mathcal{V}_{\mathbf{c}}^{0}$ :

$$
Q_{0}(z) \mathbf{A}(z) Q_{0}(z)^{-1}=\left(\begin{array}{ccc}
\omega_{1}(z) I_{n_{1}} & & \mathbf{0} \\
& \ddots & \\
\mathbf{0} & & \omega_{J}(z) I_{n_{J}}
\end{array}\right)=: D_{1}(z) .
$$

This corresponds to a diagonalization of the symbol $\mathbf{A}$ on the boundary $\partial \Omega$, when the frequencies are close to the unstable frequencies.

On the boundary $\partial \Omega$ of the space domain, the set of space-frequency variables is thus decomposed as the union of a set $\mathcal{V}_{\mathbf{c}}^{0}$ that contains all the unstable points and a set that contains only uniformly stable points. In the subsequent analysis, we shall show that the instabilities originating from the critical set $\Sigma_{\mathbf{c}}$ propagate in the interior domain along bicharacteristic curves. In order to control where these instabilities propagate, we are led to the important assumption that these bicharacteristic curves are well-defined in all the interior domain:

Assumption 7. There exists an open set $\mathcal{V}_{\mathbf{c}} \subset \bar{\Omega} \times \Sigma_{+}$satisfying

$$
\mathcal{V}_{\mathbf{c}} \cap\left\{x_{d}=0\right\}=\mathcal{V}_{\mathbf{c}}^{0}
$$

and there exists a symbol $Q_{0}$ of degree 0 and regularity 2 defined on $\mathcal{V}_{\mathbf{c}}$ such that (38) holds on all $\mathcal{V}_{\mathbf{c}}$.

Moreover, decomposing $\omega_{j}$ as $\omega_{j}=\gamma e_{j}+\mathrm{i} h_{j}$ (all mappings are defined on $\mathcal{V}_{\mathbf{c}}$ ), the solutions of the Hamiltonian ODEs system 


$$
\begin{aligned}
& \frac{\mathrm{d} x_{k}}{\mathrm{~d} x_{d}}=\frac{\partial h_{j}}{\partial \eta_{k}}(x, \eta, \gamma), \quad k=0, \ldots, d-1, \\
& \frac{\mathrm{d} \eta_{k}}{\mathrm{~d} x_{d}}=-\frac{\partial h_{j}}{\partial x_{k}}(x, \eta, \gamma), \quad k=0, \ldots, d-1, \\
& \left(x_{0}, \ldots, x_{d-1}, \eta_{0}, \ldots, \eta_{d-1}, \gamma\right)_{\left.\right|_{d}=0} \in \mathcal{V}_{\mathbf{c}}^{0}
\end{aligned}
$$

are defined for all $x_{d} \geqslant 0$, that is, stay in $\mathcal{V}_{\mathbf{c}}$ for all $x_{d} \geqslant 0$. These solutions are referred to as bicharacteristic curves.

Note that Assumption 7 is met in the constant coefficients case. Indeed, $\mathcal{V}_{\mathbf{c}}^{0}$ may be chosen as the product $\partial \Omega \times \mathcal{V}_{\text {cr }}(\mathbf{u})$, and we choose in this case

$$
\mathcal{V}_{\mathbf{c}}:=\bar{\Omega} \times \mathcal{V}_{\mathrm{cr}}(\mathbf{u})
$$

Then the mapping $Q_{0}$ exists because of Assumption 5, and it is independent of $x$, as well as the $\omega_{j}$ 's. The ODEs system (39) then reduces to

$$
\begin{aligned}
& \frac{\mathrm{d} x_{k}}{\mathrm{~d} x_{d}}=\frac{\partial h_{j}}{\partial \eta_{k}}(\eta, \gamma), \quad k=0, \ldots, d-1, \\
& \frac{\mathrm{d} \eta_{k}}{\mathrm{~d} x_{d}}=0, \quad k=0, \ldots, d-1, \\
& \left(x_{0}, \ldots, x_{d-1}, \eta_{0}, \ldots, \eta_{d-1}, \gamma\right)_{\left.\right|_{d}=0} \in \mathcal{V}_{\mathbf{c}}^{0} .
\end{aligned}
$$

Our choice of $\mathcal{V}_{\mathbf{c}}$ implies that the bicharacteristic curves stay in $\mathcal{V}_{\mathbf{c}}$ (frequencies are constant along these curves because $h_{j}$ does not depend on $x$ ).

In the variable coefficients case, we recall that the perturbation $\dot{u}=\left(\dot{u}_{r}, \dot{u}_{l}\right)$ has compact support. Consequently, if $\left(x_{k}, \eta_{k}\right)$ is a solution of (39), $\eta_{k}$ is constant for $x_{d}$ large enough. From standard ODEs arguments, we claim that Assumption 7 is satisfied when $\dot{u}$ is a sufficiently small perturbation (one can choose $\mathcal{V}_{\mathbf{c}}$ as in the constant coefficients case, provided the perturbation is small enough). We refer to Fig. 2 for a schematic picture of the situation.

For $(y, \eta, \gamma) \in \partial \Omega \times \mathbb{R}^{d} \times \mathbb{R}^{+}$, define

$$
\sigma(y, \eta, \gamma):=\tilde{\sigma}(\mathbf{a}(y), \eta, \gamma)
$$

so we have $\sigma \in \Gamma_{2}^{1}\left(\mathbb{R}^{d}\right)$.

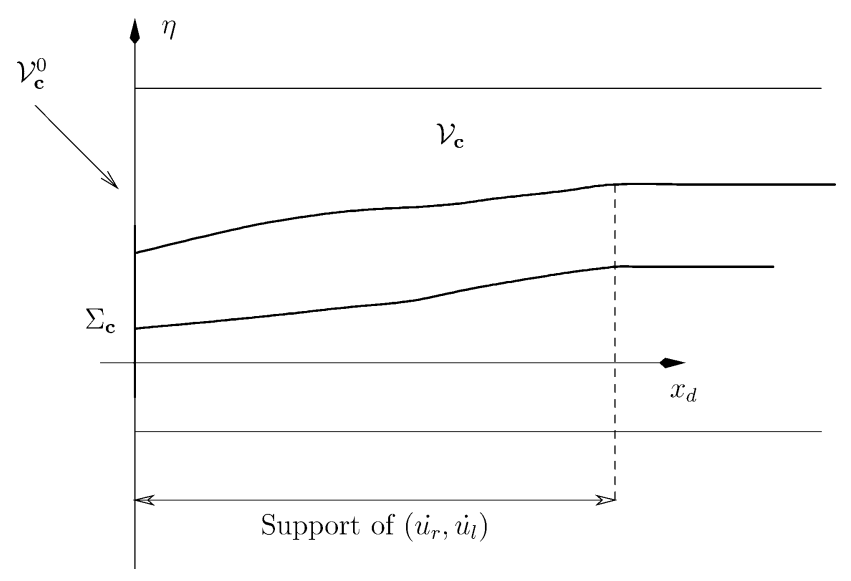

Fig. 2. Bicharacteristic curves originating from the boundary. 
With the help of Assumption 7, it is possible to construct a solution of the transport equation

$$
\begin{aligned}
& \partial_{x_{d}} \sigma_{j}+\sum_{k=0}^{d-1} \partial_{x_{k}} \sigma_{j} \partial_{\eta_{k}} h_{j}-\partial_{\eta_{k}} \sigma_{j} \partial_{x_{k}} h_{j}=0, \quad(x, \eta, \gamma) \in \mathcal{V}_{\mathbf{c}}, \\
& \sigma_{\dot{J}_{x_{d}=0}}=\sigma, \quad(x, \eta, \gamma) \in \mathcal{V}_{\mathbf{c}}^{0},
\end{aligned}
$$

and the solution $\sigma_{j}$ of this equation is homogeneous of degree 1 with respect to $(\eta, \gamma)$. The solution $\sigma_{j}$ is constant along the characteristic curves of Eq. (40), and these curves are exactly the bicharacteristic curves defined earlier.

Note that (40) also reads

$$
\partial_{x_{d}} \sigma_{j}+\left\{\sigma_{j}, h_{j}\right\}=0
$$

where $\left\{\sigma_{j}, h_{j}\right\}$ is the Poisson bracket of $\sigma_{j}$ and $h_{j}$.

For convenience, we extend all mappings $\omega_{j}, 1 \leqslant j \leqslant J$ (and thus $D_{1}$ ) as symbols of degree 1 and regularity 2 defined for all $(x, \eta, \gamma)$. We therefore have $\omega_{j} \in \Gamma_{2}^{1}$. We choose these extensions such that one has either

$$
e_{j} \geqslant c>0 \text { or } e_{j} \leqslant-c<0,
$$

where $\omega_{j}=\gamma e_{j}+\mathrm{i} h_{j}$. Extending the $\omega_{j}$ 's allows to define a global solution $\sigma_{j}$ of (40), meaning that $\sigma_{j}$ is defined for all $(x, \eta, \gamma) \in \Omega \times \Sigma_{+}$and not only for $(x, \eta, \gamma) \in \mathcal{V}_{\mathbf{c}}$. This global solution belongs to $\Gamma_{2}^{1}$. What is important is that we have not changed the value of $\sigma_{j}$ on the set $\mathcal{V}_{\mathbf{c}}$ since $\sigma_{j}$ is constant along the bicharacteristic curves. The functions $\sigma_{j}$ are weights that vanish only on the curves originating from the critical points. We shall see in the sequel that they are appropriate in the derivation of an energy estimate.

Finally, we need to precise the behavior of the restriction of the boundary symbol $\boldsymbol{\beta}$ to the stable subspace. Recall that the first $N-1$ column vectors of the matrix $Q_{0}(z)^{-1}$ span the stable subspace $\mathcal{E}^{-}(z)$ when $z \in \mathcal{V}_{\mathbf{c}}^{0}$. We write:

$$
Q_{0}(z)^{-1}=\left(Q_{\text {in }}(z) \quad Q_{\text {out }}(z)\right), \quad Q_{\text {in }}(z) \in \mathcal{M}_{2 N, N-1}(\mathbb{C}),
$$

and make the following assumption:

Assumption 8. There exist two mappings $P_{1}$ and $P_{2}$ defined on $\mathcal{V}_{\mathbf{c}}^{0}$ such that

for all $z \in \mathcal{V}_{\mathbf{c}}^{0}, P_{1}(z) \in G l_{N-1}(\mathbb{C})$ and $P_{1}$ is a symbol of degree 0 and regularity 1 , for all $z \in \mathcal{V}_{\mathbf{c}}^{0}, P_{2}(z) \in G l_{N-1}(\mathbb{C})$ and $P_{2}$ is a symbol of degree 0 and regularity 1 , for all $z \in \mathcal{V}_{\mathbf{c}}^{0}$, one has

$$
P_{1}(z) \boldsymbol{\beta}(z) Q_{\text {in }}(z) P_{2}(z)=\left(\begin{array}{cc}
\lambda^{-1, \gamma}(\eta)(\gamma+\mathrm{i} \sigma(z)) & 0 \\
0 & I_{N-2}
\end{array}\right)=: \boldsymbol{\beta}_{\text {in }}(z) .
$$

In the constant coefficients case, Assumption 8 implies (18). The meaning of Assumption 8 is that the restriction of $\boldsymbol{\beta}$ to the stable subspace has a kernel of dimension 1 (this was not part of Assumption 5) and " $\boldsymbol{\beta}$ vanishes at order 1 on this kernel". To check Assumption 8 in practice, it is sufficient to check it for a planar shock wave. Using Assumption 6, a similar reduction will hold for a perturbed planar shock satisfying the Rankine-Hugoniot relations.

With these preliminary reductions in mind, we can turn to the derivation of our energy estimates. We fix a nonnegative cut-off function $\chi$ verifying

$\chi$ is a smooth (that is, $\mathcal{C}^{\infty}$ ) symbol of degree 0 and Supp $\chi \subset \mathcal{V}_{\mathbf{c}}$,

$\chi \equiv 1$ in a neighborhood of the bicharacteristic curves originating from $\Sigma_{\mathbf{c}}$. In other words, out of the region where $\chi \equiv 1$, one has $\left|\sigma_{j}\right| \geqslant c>0$ for all $j$.

Define $\chi_{0}:=1-\chi$ and observe that $\chi_{0}$ has its support in the set of uniformly stable points. 


\subsection{Energy estimates near instability points}

The aim of this paragraph is to derive three different energy estimates in the neighborhood of instability points. We show how to control the $L^{2}\left(H^{1}\right)$ norm of the outgoing modes. For the incoming modes, we show how to control the $L^{2}$ norm and the $L^{2}\left(H^{1}\right)$ norm far from the bicharacteristic curves starting from the critical set $\Sigma_{\mathbf{c}}$.

Before establishing our main energy estimates, we prove a reduction result that will help us to deal with the zero order term in the linearized equations. Recall that relation (38) holds on all the open set $\mathcal{V}_{\mathbf{c}}$ and not only on its trace $\mathcal{V}_{\mathbf{c}}^{0}$. In terms of symbolic calculus, we are looking for a symbol $Q_{-1}$ of degree -1 and regularity 1 such that

$$
\left(Q_{0}+Q_{-1}\right) \#\left(\partial_{d}-\mathbf{A}-\mathbf{A}_{d}^{-1} \mathbf{C}\right)=\left(\partial_{d}-D_{1}-D_{0}\right) \#\left(Q_{0}+Q_{-1}\right)
$$

where the composition of symbols is to be understood as the expansion to first or second order (depending on the regularity with respect to $x$ ), see Theorem A.2. Recall that our symbols have finite smoothness in the space variable so the expansions of adjoints or composed symbols are only finite and not asymptotic.

The existence of $Q_{-1}$ is given by the following lemma:

Lemma 1. Let $Q_{0}$ be defined by Assumption 7, and define $D_{1}$ as in (38). There exists a symbol $Q_{-1}$ of degree -1 and regularity 1 , defined on $\mathcal{V}_{\mathbf{c}}$, such that

$$
\left(Q_{0}+Q_{-1}\right)\left(\mathbf{A}+\mathbf{A}_{d}^{-1} \mathbf{C}\right)+\partial_{d} Q_{0}+\frac{1}{\mathrm{i}} \sum_{k=0}^{d-1}\left(\partial_{\eta_{k}} Q_{0} \partial_{x_{k}} \mathbf{A}-\partial_{\eta_{k}} D_{1} \partial_{x_{k}} Q_{0}\right)-\left(D_{1}+D_{0}\right)\left(Q_{0}+Q_{-1}\right)
$$

is a symbol of degree -1 and regularity 1 , and $D_{0}$ is a block diagonal symbol (of degree 0 and regularity 1) whose blocks have dimensions $n_{1}, \ldots, n_{J}$ as those of $D_{1}$.

Proof. Using the equality $Q_{0} \mathbf{A}=D_{1} Q_{0}$, the problem reduces to finding a symbol $Q_{-1}$ of degree -1 such that

$$
\left[Q_{-1} Q_{0}^{-1}, D_{1}\right]+Q_{0} \mathbf{A}_{d}^{-1} \mathbf{C} Q_{0}^{-1}+\partial_{d} Q_{0}+\frac{1}{\mathrm{i}} \sum_{k=0}^{d-1} \partial_{\eta_{k}} Q_{0} \partial_{x_{k}} \mathbf{A}-\partial_{\eta_{k}} D_{1} \partial_{x_{k}} Q_{0}
$$

is block diagonal (it will automatically be a symbol of degree 0 ). Here above $[M, N]$ denotes the commutator of two matrices $M$ and $N$. Using that $D_{1}$ is block diagonal (with diagonal blocks $\omega_{1} I_{n_{1}}, \ldots, \omega_{J} I_{n_{J}}$ ), a simple calculation shows that one can choose $Q_{-1} Q_{0}^{-1}$ (and therefore $Q_{-1}$ ) such that the extra diagonal blocks of $\left[Q_{-1} Q_{0}^{-1}, D_{1}\right]$ cancel those of

$$
Q_{0} \mathbf{A}_{d}^{-1} \mathbf{C} Q_{0}^{-1}+\partial_{d} Q_{0}+\frac{1}{\mathrm{i}} \sum_{k=0}^{d-1} \partial_{\eta_{k}} Q_{0} \partial_{x_{k}} \mathbf{A}-\partial_{\eta_{k}} D_{1} \partial_{x_{k}} Q_{0}
$$

Because the diagonal blocks of $\left[Q_{-1} Q_{0}^{-1}, D_{1}\right]$ are identically zero, one can only cancel the extra diagonal blocks. It stems from this simple calculation that $Q_{-1} Q_{0}^{-1}$ is of degree -1 and regularity 1 , and therefore so is $Q_{-1}$. This proves the lemma.

Note that $Q_{-1}$ and $D_{0}$ are only defined for space-frequency variables belonging to $\mathcal{V}_{\mathbf{c}}$ but, as was done for $D_{1}$, we extend $D_{0}$ as a global symbol of degree 0 and regularity 1 .

In all the sequel, we denote by $Q$ the sum $Q_{0}+Q_{-1}$. The following calculations heavily use the fact that $Q$ defines a "good diagonalization basis" of the paralinearized operator

$$
\dot{v} \mapsto \partial_{d} \dot{v}-T_{\mathbf{A}}^{\gamma} \dot{v}-T_{\mathbf{A}_{d}^{-1} \mathbf{C}}^{\gamma} \dot{v}
$$

Let $\dot{v} \in H^{2}(\Omega)$ and define

$$
F:=\partial_{d} \dot{v}-T_{\mathbf{A}}^{\gamma} \dot{v}-T_{\mathbf{A}_{d}^{-1} \mathbf{C}}^{\gamma} \dot{v} \in H^{1}(\Omega) .
$$


We also define

$$
w:=T_{\chi Q}^{\gamma} \dot{v}
$$

and we first show that $w$ satisfies a paradifferential equation whose first and zero order symbol are block diagonal. The paradifferential equation involves error terms that will be absorbed at the very end of the analysis. In all this paragraph, $R^{\gamma}$ always denotes an operator of order $\leqslant-1$ that represents the current error terms in the computations. We have

$$
\begin{aligned}
\partial_{d} w & =T_{\left(\partial_{d} \chi\right) Q}^{\gamma} \dot{v}+T_{\chi \partial_{d} Q}^{\gamma} \dot{v}+T_{\chi Q}^{\gamma}\left(\partial_{d} \dot{v}\right) \\
& =T_{\left(\partial_{d} \chi\right) Q}^{\gamma} \dot{v}+T_{\chi \partial_{d} Q}^{\gamma} \dot{v}+T_{\chi Q}^{\gamma}\left(T_{\mathbf{A}}^{\gamma} \dot{v}+T_{\mathbf{A}_{d}^{-1} \mathbf{C}}^{\gamma} \dot{v}+F\right) \\
& =T_{\left(\partial_{d} \chi\right) Q_{0}}^{\gamma} \dot{v}+T_{\chi \partial_{d} Q_{0}}^{\gamma} \dot{v}+T_{\chi Q\left(\mathbf{A}+\mathbf{A}_{d}^{-1} \mathbf{C}\right)}^{\gamma} \dot{v}+T_{r^{1}}^{\gamma} \dot{v}+R^{\gamma} \dot{v}+T_{\chi Q}^{\gamma} F,
\end{aligned}
$$

where

$$
r^{1}:=\frac{1}{\mathrm{i}} \sum_{k=0}^{d-1} \partial_{\eta_{k}}\left(\chi Q_{0}\right) \partial_{x_{k}} \mathbf{A} .
$$

Lemma 1 implies that

$$
\chi \partial_{d} Q_{0}+\chi Q\left(\mathbf{A}+\mathbf{A}_{d}^{-1} \mathbf{C}\right)+r^{1}-\left(\left(D_{1}+D_{0}\right) \chi Q+\frac{1}{\mathrm{i}} \sum_{k=0}^{d-1}\left(\partial_{\eta_{k}} \chi\right) Q_{0} \partial_{x_{k}} \mathbf{A}+\chi\left(\partial_{\eta_{k}} D_{1}\right) \partial_{x_{k}} Q_{0}\right)
$$

is of degree -1 , and we thus get

$$
\partial_{d} w=T_{\left(\partial_{d} \chi\right) Q_{0}}^{\gamma} \dot{v}+\left(T_{D_{1}}^{\gamma}+T_{D_{0}}^{\gamma}\right) w+T_{r^{2}}^{\gamma} \dot{v}+T_{r^{3}}^{\gamma} \dot{v}+R^{\gamma} \dot{v}+T_{\chi Q}^{\gamma} F,
$$

where

$$
\begin{aligned}
r^{2} & :=\frac{1}{\mathrm{i}} \sum_{k=0}^{d-1}\left(\partial_{\eta_{k}} \chi\right) Q_{0} \partial_{x_{k}} \mathbf{A}+\chi\left(\partial_{\eta_{k}} D_{1}\right) \partial_{x_{k}} Q_{0}, \\
r^{3} & :=-\frac{1}{\mathrm{i}} \sum_{k=0}^{d-1}\left(\partial_{\eta_{k}} D_{1}\right) \partial_{x_{k}}\left(\chi Q_{0}\right) .
\end{aligned}
$$

After simplifying $r^{2}+r^{3}$, we get the relation

$$
\partial_{d} w=T_{D_{1}}^{\gamma} w+T_{D_{0}}^{\gamma} w+T_{r}^{\gamma} \dot{v}+R^{\gamma} \dot{v}+T_{\chi Q}^{\gamma} F,
$$

with

$$
r:=\left(\partial_{d} \chi\right) Q_{0}+\frac{1}{\mathrm{i}} \sum_{k=0}^{d-1}\left(\partial_{\eta_{k}} \chi\right) Q_{0} \partial_{x_{k}} \mathbf{A}-\left(\partial_{x_{k}} \chi\right)\left(\partial_{\eta_{k}} D_{1}\right) Q_{0} .
$$

As a consequence, $r$ is of order 0 and is identically zero in the domain where $\chi \equiv 1$. Therefore $r$ has its support far from the "unstable" points.

Recall that $D_{1}$ is diagonal and $D_{0}$ is block diagonal:

$$
D_{1}=\left(\begin{array}{ccc}
\omega_{1} I_{n_{1}} & & \\
& \ddots & \\
& & \omega_{J} I_{n_{J}}
\end{array}\right), \quad D_{0}=\left(\begin{array}{ccc}
C_{1} & & \\
& \ddots & \\
& & C_{J}
\end{array}\right),
$$

so (42) can be written as a collection of $J$ equations

$$
\partial_{d} w_{j}=T_{\omega_{j}}^{\gamma} w_{j}+T_{C_{j}}^{\gamma} w_{j}+T_{r_{j}}^{\gamma} \dot{v}+R^{\gamma} \dot{v}+T_{\chi Q_{j}}^{\gamma} F,
$$

with $\operatorname{Re} \omega_{j}<0$ when $\gamma>0$ and $1 \leqslant j \leqslant J^{\prime}$, and $\operatorname{Re} \omega_{j}>0$ when $\gamma>0$ and $J^{\prime}+1 \leqslant j \leqslant J$. 


\subsubsection{Estimate for the outgoing modes}

We first deal with the indexes $j$ for which $\operatorname{Re} \omega_{j}>0$ when $\gamma>0$. Recall that $\omega_{j}$ is defined for all $(\eta, \gamma)$ and satisfies

$$
\forall(\eta, \gamma) \in \mathbb{R}^{d} \times \mathbb{R}^{+}, \quad \operatorname{Re} \omega_{j} \geqslant c \gamma .
$$

We choose $\Lambda^{2, \gamma}$ as a symmetrizer for (44), where $\Lambda^{2, \gamma}$ is the Fourier multiplier of symbol $\lambda^{2, \gamma}(\eta)$. Taking the scalar product in $L^{2}(\Omega)$ of (44) with $\Lambda^{2, \gamma} w_{j}$, we get

$$
\begin{aligned}
-\left\|w_{j}(0)\right\|_{1, \gamma}^{2}= & 2 \operatorname{Re}\left\|\Lambda^{1, \gamma} T_{\omega_{j}}^{\gamma} w_{j}, \Lambda^{1, \gamma} w_{j}\right\|_{L^{2}(\Omega)}+2 \operatorname{Re}\left\|\Lambda^{1, \gamma} T_{C_{j}}^{\gamma} w_{j}, \Lambda^{1, \gamma} w_{j}\right\|_{L^{2}(\Omega)} \\
& +2 \operatorname{Re}\left\|\Lambda^{1, \gamma} T_{r_{j}}^{\gamma} \dot{v}, \Lambda^{1, \gamma} w_{j}\right\|_{L^{2}(\Omega)}+2 \operatorname{Re}\left\langle\Lambda^{1, \gamma} R^{\gamma} \dot{v}, \Lambda^{1, \gamma} w_{j} \|_{L^{2}(\Omega)}\right. \\
& +2 \operatorname{Re}\left\|\Lambda^{1, \gamma} T_{\chi Q_{j}}^{\gamma} F, \Lambda^{1, \gamma} w_{j}\right\|_{L^{2}(\Omega)} .
\end{aligned}
$$

Taking the order of the different operators into account and using Young's inequality, we obtain

$$
\begin{aligned}
& -2 \operatorname{Re}\left\|\Lambda^{1, \gamma} T_{C_{j}}^{\gamma} w_{j}, \Lambda^{1, \gamma} w_{j}\right\|_{L^{2}(\Omega)} \leqslant C\left\|w_{j}\right\|_{1, \gamma}^{2}, \\
& -2 \operatorname{Re}\left\|\Lambda^{1, \gamma} T_{r_{j}}^{\gamma} \dot{v}, \Lambda^{1, \gamma} w_{j}\right\|_{L^{2}(\Omega)} \leqslant \frac{C}{\gamma}\left\|T_{r_{j}}^{\gamma} \dot{v}\right\|_{1, \gamma}^{2}+\varepsilon \gamma\left\|w_{j}\right\|_{1, \gamma}^{2}, \\
& -2 \operatorname{Re}\left\|\Lambda^{1, \gamma} R^{\gamma} \dot{v}, \Lambda^{1, \gamma} w_{j}\right\|_{L^{2}(\Omega)} \leqslant \frac{C}{\gamma}\|\dot{v}\|_{0}^{2}+\varepsilon \gamma\left\|w_{j}\right\|_{1, \gamma}^{2}, \\
& -2 \operatorname{Re}\left\|\Lambda^{1, \gamma} T_{\chi Q_{j}}^{\gamma} F, \Lambda^{1, \gamma} w_{j}\right\|_{L^{2}(\Omega)} \leqslant \frac{C}{\gamma}\left\|T_{\chi Q}^{\gamma} F\right\|_{1, \gamma}^{2}+\varepsilon \gamma\left\|w_{j}\right\|_{1, \gamma}^{2},
\end{aligned}
$$

for some $\varepsilon>0$ to be fixed. Using Theorem A.2, the difference

$$
\Lambda^{1, \gamma} T_{\omega_{j}}^{\gamma}-T_{\omega_{j}}^{\gamma} \Lambda^{1, \gamma}
$$

is of order $\leqslant 1$ so we have

$$
2 \operatorname{Re}\left\langle\left\langle\Lambda^{1, \gamma} T_{\omega_{j}}^{\gamma} w_{j}, \Lambda^{1, \gamma} w_{j}\left\|_{L^{2}(\Omega)} \geqslant 2 \operatorname{Re}\right\| T_{\omega_{j}}^{\gamma} \Lambda^{1, \gamma} w_{j}, \Lambda^{1, \gamma} w_{j}\left\|_{L^{2}(\Omega)}-C\right\| w_{j} \|_{1, \gamma}^{2} .\right.\right.
$$

Applying Gårding's inequality (Theorem A.3), we finally get

$$
2 \operatorname{Re}\left\|\Lambda^{1, \gamma} T_{\omega_{j}}^{\gamma} w_{j}, \Lambda^{1, \gamma} w_{j}\right\|_{L^{2}(\Omega)} \geqslant(c \gamma-C)\left\|w_{j}\right\|_{1, \gamma}^{2} .
$$

It is now clear that an appropriate choice of $\varepsilon$ yields the $L^{2}\left(H^{1}\right)$ estimate

$$
\gamma\left\|w_{j}\right\|_{1, \gamma}^{2}+\left\|w_{j}(0)\right\|_{1, \gamma}^{2} \lesssim \frac{1}{\gamma}\left\|T_{\chi Q}^{\gamma} F\right\|_{1, \gamma}^{2}+\frac{1}{\gamma}\left(\|\dot{v}\|_{0}^{2}+\left\|T_{r}^{\gamma} \dot{v}\right\|_{1, \gamma}^{2}\right) .
$$

\subsubsection{Estimate for the incoming modes}

We now deal with the indexes $j$ for which $\operatorname{Re} \omega_{j}<0$ when $\gamma>0$ (and therefore $\operatorname{Re} \omega_{j} \leqslant-c \gamma$ ). We first choose the identity as a symmetrizer and perform the same computation as above. One can indeed proceed in a similar way because the symmetrizer is a constant coefficients operator (that is, a Fourier multiplier). Because of the sign of $\operatorname{Re} \omega_{j}$, we have

$$
\gamma\left\|w_{j}\right\|_{0}^{2} \lesssim\left\|w_{j}(0)\right\|_{0}^{2}+\frac{1}{\gamma^{3}}\left\|T_{\chi Q}^{\gamma} F\right\|_{1, \gamma}^{2}+\frac{1}{\gamma^{3}}\left(\|\dot{v}\|_{0}^{2}+\|\| T_{r_{j}}^{\gamma} \dot{v} \|_{1, \gamma}^{2}\right),
$$

and we rewrite this estimate as

$$
\gamma^{3}\left\|w_{j}\right\|_{0}^{2} \lesssim \gamma^{2}\left\|w_{j}(0)\right\|_{0}^{2}+\frac{1}{\gamma}\left\|T_{\chi Q}^{\gamma} F\right\|_{1, \gamma}^{2}+\frac{1}{\gamma}\left(\|\dot{v}\|_{0}^{2}+\left\|T_{r}^{\gamma} \dot{v}\right\|_{1, \gamma}^{2}\right) .
$$


The right-hand terms in (45) and (46) have similar expressions. The only difference is that the boundary value of $w_{j}$ is on the right-hand side of the inequality when we deal with an incoming mode.

At this stage, it is important to note that both right-hand side terms in (45) and (46) involve the $L^{2}\left(H^{1}\right)$ norm of $T_{r}^{\gamma} \dot{v}$, and $r$ is identically zero in the region $\{\chi \equiv 1\}$, see (43). In particular, $r$ is identically zero near the bicharacteristic curves originating from $\Sigma_{\mathbf{c}}$. However, $r$ is not identically zero in $\mathcal{V}_{\mathbf{c}}$ so, in order to absorb the error term $\left\|T_{r}^{\gamma} \dot{v}\right\|_{1, \gamma}$, we need to control the $L^{2}\left(H^{1}\right)$ norm of the $w_{j}$ 's in $\mathcal{V}_{\mathbf{c}}$ far from the bicharacteristic curves. We are thus going to estimate $T_{\sigma_{j}}^{\gamma} w_{j}$.

We choose $S_{j}:=\left(T_{\sigma_{j}}^{\gamma}\right)^{*} T_{\sigma_{j}}^{\gamma}$ as a symmetrizer for (44). Recall that $S_{j}$ is of order $\leqslant 2$ since $\sigma_{j} \in \Gamma_{2}^{1}$. We take the scalar product in $L^{2}(\Omega)$ of (44) with $S_{j} w_{j}$. This yields

$$
\begin{aligned}
-\left\|T_{\sigma}^{\gamma} w_{j}(0)\right\|_{0}^{2}= & \operatorname{Re}\left\langle\left(\partial_{d} S_{j}\right) w_{j}, w_{j} \|_{L^{2}(\Omega)}+2 \operatorname{Re}\left\langle\left\langle S_{j} T_{\omega_{j}}^{\gamma} w_{j}, w_{j} \|_{L^{2}(\Omega)}+2 \operatorname{Re}\left\langle S_{j} T_{C_{j}}^{\gamma} w_{j}, w_{j} \|_{L^{2}(\Omega)}\right.\right.\right.\right. \\
& +2 \operatorname{Re}\left\langle\left\langle S_{j} T_{r_{j}}^{\gamma} \dot{v}, w_{j} \|_{L^{2}(\Omega)}+2 \operatorname{Re}\left\langle\left\langle S_{j} R^{\gamma} \dot{v}, w_{j} \|_{L^{2}(\Omega)}+2 \operatorname{Re}\left\langle\left\langle S_{j} T_{\chi Q_{j}}^{\gamma} F, w_{j} \|_{L^{2}(\Omega)} .\right.\right.\right.\right.\right.\right.
\end{aligned}
$$

First observe that

$$
\partial_{d} S_{j}=\left(T_{\partial_{d} \sigma_{j}}^{\gamma}\right)^{*} T_{\sigma_{j}}^{\gamma}+\left(T_{\sigma_{j}}^{\gamma}\right)^{*} T_{\partial_{d} \sigma_{j}}^{\gamma},
$$

so we have

$$
\operatorname{Re}\left\langle\left(\partial_{d} S_{j}\right) w_{j}, w_{j} \|_{L^{2}(\Omega)}=2 \operatorname{Re}\left\langle T_{\partial_{d} \sigma_{j}}^{\gamma} w_{j}, T_{\sigma_{j}}^{\gamma} w_{j} \|_{L^{2}(\Omega)} .\right.\right.
$$

We recall that $\sigma_{j} \in \mathbb{R}$, hence the difference

$$
T_{\sigma_{j}}^{\gamma} T_{C_{j}}^{\gamma}-T_{C_{j}}^{\gamma} T_{\sigma_{j}}^{\gamma}
$$

is of order $\leqslant 0$, and we get

$$
\begin{aligned}
2 \operatorname{Re}\left\|T_{\sigma_{j}}^{\gamma} T_{C_{j}}^{\gamma} w_{j}, T_{\sigma_{j}}^{\gamma} w_{j}\right\|_{L^{2}(\Omega)} & \leqslant C\left\|T_{\sigma_{j}}^{\gamma} w_{j}\right\|_{0}^{2}+C\left\|w_{j}\right\|_{0}\left\|T_{\sigma_{j}}^{\gamma} w_{j}\right\| \\
& \leqslant C\left\|T_{\sigma_{j}}^{\gamma} w_{j}\right\|_{0}^{2}+\frac{C}{\gamma}\left\|w_{j}\right\|_{0}^{2}+\varepsilon \gamma\left\|T_{\sigma_{j}}^{\gamma} w_{j}\right\|_{0}^{2},
\end{aligned}
$$

for some $\varepsilon>0$ to be fixed. Because $T_{\sigma_{j}}^{\gamma}$ is of order $\leqslant 1$, Young's inequality yields

$$
\begin{aligned}
& 2 \operatorname{Re}\left\langle T_{\sigma_{j}}^{\gamma} T_{r_{j}}^{\gamma} \dot{v}, T_{\sigma_{j}}^{\gamma} w_{j}\left\|_{L^{2}(\Omega)} \leqslant \frac{C}{\gamma}\right\| T_{r_{j}}^{\gamma} \dot{v}\left\|_{1, \gamma}^{2}+\varepsilon \gamma\right\| T_{\sigma_{j}}^{\gamma} w_{j} \|_{0}^{2},\right. \\
& 2 \operatorname{Re}\left\langle T_{\sigma_{j}}^{\gamma} R^{\gamma} \dot{v}, T_{\sigma_{j}}^{\gamma} w_{j}\left\|_{L^{2}(\Omega)} \leqslant \frac{C}{\gamma}\right\| \dot{v}\left\|_{0}^{2}+\varepsilon \gamma\right\| T_{\sigma_{j}}^{\gamma} w_{j} \|_{0}^{2},\right. \\
& 2 \operatorname{Re}\left\langle\left\langle T_{\sigma_{j}}^{\gamma} T_{\chi Q_{j}}^{\gamma} F, T_{\sigma_{j}}^{\gamma} w_{j}\left\|_{L^{2}(\Omega)} \leqslant \frac{C}{\gamma}\right\| T_{\chi Q}^{\gamma} F\left\|_{1, \gamma}^{2}+\varepsilon \gamma\right\| T_{\sigma_{j}}^{\gamma} w_{j} \|_{0}^{2} .\right.\right.
\end{aligned}
$$

Collecting these first inequalities, we already have

$$
\begin{aligned}
& -2 \operatorname{Re}\left\|T_{\partial_{d} \sigma_{j}}^{\gamma} w_{j}+T_{\sigma_{j}}^{\gamma} T_{\omega_{j}}^{\gamma} w_{j}, T_{\sigma_{j}}^{\gamma} w_{j}\right\|_{L^{2}(\Omega)} \\
& \quad \leqslant\left\|T_{\sigma}^{\gamma} w_{j}(0)\right\|_{0}^{2}+(C+4 \varepsilon \gamma)\left\|T_{\sigma_{j}}^{\gamma} w_{j}\right\|_{0}^{2}+\frac{C}{\gamma}\left\|T_{\chi Q}^{\gamma} F\right\|_{1, \gamma}^{2}+\frac{C}{\gamma}\left\|w_{j}\right\|_{0}^{2}+\frac{C}{\gamma}\left(\|\dot{v}\|_{0}^{2}+\left\|T_{r}^{\gamma} \dot{v}\right\|_{1, \gamma}^{2}\right) .
\end{aligned}
$$

We are now going to derive a lower bound for the left-hand term in (47). Write $\omega_{j}=\gamma e_{j}+\mathrm{i} h_{j}$ with $e_{j}, h_{j} \in \mathbb{R}$ and $e_{j} \in \Gamma_{2}^{0}, h_{j} \in \Gamma_{2}^{1}$. From Theorem A.2, we have

$$
T_{\sigma_{j}}^{\gamma} T_{\omega_{j}}^{\gamma}=\gamma T_{e_{j}}^{\gamma} T_{\sigma_{j}}^{\gamma}+\gamma T_{-\mathrm{i}\left\{\sigma_{j}, e_{j}\right\}}^{\gamma}+\gamma R^{\gamma}+T_{\mathrm{i} h_{j}}^{\gamma} T_{\sigma_{j}}^{\gamma}+T_{\left\{\sigma_{j}, h_{j}\right\}}^{\gamma}+R_{0}^{\gamma}
$$


where $R^{\gamma}$ still denotes an operator of order $\leqslant-1$ and $R_{0}^{\gamma}$ denotes an operator of order $\leqslant 0$. Recall that $\sigma_{j}$ is a solution to the transport equation

$$
\left\{\begin{array}{l}
\partial_{d} \sigma_{j}+\left\{\sigma_{j}, h_{j}\right\}=0, \quad x_{d}>0, \\
\sigma_{\left.\right|_{\left.\right|_{d}=0}}=\sigma,
\end{array}\right.
$$

so we get

$$
\begin{aligned}
-2 & \operatorname{Re}\left\langle\left\langle T_{\partial_{d} \sigma_{j}}^{\gamma} w_{j}+T_{\sigma_{j}}^{\gamma} T_{\omega_{j}}^{\gamma} w_{j}, T_{\sigma_{j}}^{\gamma} w_{j} \|_{L^{2}(\Omega)}\right.\right. \\
= & -2 \gamma \operatorname{Re}\left\langle T_{e_{j}}^{\gamma} T_{\sigma_{j}}^{\gamma} w_{j}, T_{\sigma_{j}}^{\gamma} w_{j} \|_{L^{2}(\Omega)}-2 \gamma \operatorname{Re}\left\langle T_{-\mathrm{i}\left\{\sigma_{j}, e_{j}\right\}}^{\gamma} w_{j}, T_{\sigma_{j}}^{\gamma} w_{j} \|_{L^{2}(\Omega)}-2 \gamma \operatorname{Re}\left\langle\left\langle R^{\gamma} w_{j}, T_{\sigma_{j}}^{\gamma} w_{j} \|_{L^{2}(\Omega)}\right.\right.\right.\right. \\
& -2\left\|\left(\operatorname{Re} T_{\mathrm{i} h_{j}}^{\gamma}\right) T_{\sigma_{j}}^{\gamma} w_{j}, T_{\sigma_{j}}^{\gamma} w_{j}\right\|_{L^{2}(\Omega)}-2 \operatorname{Re}\left\langle\left\langle R_{0}^{\gamma} w_{j}, T_{\sigma_{j}}^{\gamma} w_{j} \|_{L^{2}(\Omega)} .\right.\right.
\end{aligned}
$$

Let us first examine the last three terms of the right-hand side. Because $\mathrm{i} h_{j} \in \mathrm{i} \mathbb{R}$, the operator $\operatorname{Re} T_{i h_{j}}^{\gamma}$ is of order $\leqslant 0$ and we have

$$
\begin{aligned}
& -2\left\|\left(\operatorname{Re} T_{\mathrm{i} h_{j}}^{\gamma}\right) T_{\sigma_{j}}^{\gamma} w_{j}, T_{\sigma_{j}}^{\gamma} w_{j}\right\|_{L^{2}(\Omega)} \geqslant-C\left\|T_{\sigma_{j}}^{\gamma} w_{j}\right\|_{0}^{2}, \\
& -2 \operatorname{Re}\left\langle\left\langle R_{0}^{\gamma} w_{j}, T_{\sigma_{j}}^{\gamma} w_{j}\left\|_{L^{2}(\Omega)} \geqslant-C\right\| w_{j}\|0\| T_{\sigma_{j}}^{\gamma} w_{j} \|_{0},\right.\right. \\
& -2 \gamma \operatorname{Re}\left\langle R^{\gamma} w_{j}, T_{\sigma_{j}}^{\gamma} w_{j}\left\|_{L^{2}(\Omega)} \geqslant-C \gamma\right\| w_{j}\|\|_{-1, \gamma}\left\|T_{\sigma_{j}}^{\gamma} w_{j}\right\|_{0} \geqslant-C\left\|w_{j}\right\|\left\|_{0}\right\| T_{\sigma_{j}}^{\gamma} w_{j} \|_{0} .\right.
\end{aligned}
$$

Applying Gårding's inequality (Theorem A.3), we obtain

$$
-2 \gamma \operatorname{Re}\left\langle T_{e_{j}}^{\gamma} T_{\sigma_{j}}^{\gamma} w_{j}, T_{\sigma_{j}}^{\gamma} w_{j}\left\|_{L^{2}(\Omega)} \geqslant c \gamma\right\| T_{\sigma_{j}}^{\gamma} w_{j} \|_{0}^{2}\right.
$$

so it only remains to derive a lower bound for the term

$$
-2 \gamma \operatorname{Re}\left\langle\left\langle T_{-\mathrm{i}\left\{\sigma_{j}, e_{j}\right\}}^{\gamma} w_{j}, T_{\sigma_{j}}^{\gamma} w_{j} \|_{L^{2}(\Omega)} .\right.\right.
$$

Because $\sigma_{j} \in \Gamma_{2}^{1}$, the operator $T_{-\mathrm{i}\left\{\sigma_{j}, e_{j}\right\}}^{\gamma}$ is of order $\leqslant 0$ and therefore

$$
\begin{aligned}
-2 \gamma \operatorname{Re}\left\langle T_{-\mathrm{i}\left\{\sigma_{j}, e_{j}\right\}}^{\gamma} w_{j}, T_{\sigma_{j}}^{\gamma} w_{j} \|_{L^{2}(\Omega)}\right. & \geqslant-C \gamma\left\|w_{j}\right\|_{0}\left\|T_{\sigma_{j}}^{\gamma} w_{j}\right\|_{0} \\
& \geqslant-\varepsilon \gamma\left\|T_{\sigma_{j}}^{\gamma} w_{j}\right\|_{0}^{2}-C \gamma\left\|w_{j}\right\|_{0}^{2} .
\end{aligned}
$$

Choosing $\varepsilon$ appropriately and taking the sum of (47)-(50), we get the second estimate for the incoming modes:

$$
\gamma\left\|T_{\sigma_{j}}^{\gamma} w_{j}\right\|_{0}^{2} \lesssim\left\|T_{\sigma}^{\gamma} w_{j}(0)\right\|_{0}^{2}+\frac{1}{\gamma}\left\|T_{\chi Q}^{\gamma} F\right\|_{1, \gamma}^{2}+\gamma\left\|w_{j}\right\|_{0}^{2}+\frac{1}{\gamma}\left(\|\dot{v}\|_{0}^{2}+\left\|T_{r}^{\gamma} \dot{v}\right\|_{1, \gamma}^{2}\right) .
$$

Take the sum of (46) and (51). Choosing $\gamma$ large enough, we have

$$
\gamma^{3}\left\|w_{j}\right\|_{0}^{2}+\gamma\left\|T_{\sigma_{j}}^{\gamma} w_{j}\right\|_{0}^{2} \lesssim \gamma^{2}\left\|w_{j}(0)\right\|_{0}^{2}+\left\|T_{\sigma}^{\gamma} w_{j}(0)\right\|_{0}^{2}+\frac{1}{\gamma}\left\|T_{\chi Q}^{\gamma} F\right\|_{1, \gamma}^{2}+\frac{1}{\gamma}\left(\|\dot{v}\|_{0}^{2}+\left\|T_{r}^{\gamma} \dot{v}\right\|_{1, \gamma}^{2}\right) .
$$

Let us decompose the vector $w$ in

$$
w=\left(\begin{array}{c}
w_{\text {in }} \\
w_{\text {out }}
\end{array}\right), \quad w_{\text {in }} \in \mathbb{C}^{N-1}, \quad w_{\text {out }} \in \mathbb{C}^{N+1} .
$$

The vector $w_{\text {in }}$ is the collection of the $w_{j}$ 's that correspond to incoming modes and $w_{\text {out }}$ is the collection of the $w_{j}$ 's that correspond to outgoing modes. Taking the sum of (45) and (52), we obtain

$$
\begin{aligned}
& \gamma\left\|w_{\text {out }}\right\|_{1, \gamma}^{2}+\gamma^{3}\left\|w_{\text {in }}\right\|_{0}^{2}+\sum_{\text {incoming }} \gamma\left\|T_{\sigma_{j}}^{\gamma} w_{j}\right\|_{0}^{2}+\left\|w_{\text {out }}(0)\right\|_{1, \gamma}^{2} \\
& \lesssim \gamma^{2}\left\|w_{\text {in }}(0)\right\|_{0}^{2}+\left\|T_{\sigma}^{\gamma} w_{\text {in }}(0)\right\|_{0}^{2}+\frac{1}{\gamma}\left\|T_{\chi Q}^{\gamma} F\right\|_{1, \gamma}^{2}+\frac{1}{\gamma}\left(\|\dot{v}\|_{0}^{2}+\left\|T_{r}^{\gamma} \dot{v}\right\|_{1, \gamma}^{2}\right),
\end{aligned}
$$


and we want to show an estimate of the type

$$
\gamma^{2}\left\|w_{\text {in }}(0)\right\|_{0}^{2}+\left\|T_{\sigma}^{\gamma} w_{\text {in }}(0)\right\|_{0}^{2} \lesssim\|G\|_{1, \gamma}^{2}+\left\|w_{\text {out }}(0)\right\|_{1, \gamma}^{2},
$$

where

$$
G:=T_{\beta}^{\gamma} \dot{v}(0)
$$

\subsubsection{Estimate for the boundary terms}

We fix four cut-off functions $\chi_{1}, \chi_{2}, \chi_{3}, \chi_{4}$ such that

$$
\begin{aligned}
& \chi_{1} \equiv 1 \text { on a neighborhood of } \operatorname{Supp} \chi \cap\left\{x_{d}=0\right\}, \\
& \chi_{2} \equiv 1 \text { on a neighborhood of } \operatorname{Supp} \chi_{1}, \\
& \chi_{3} \equiv 1 \text { on a neighborhood of } \operatorname{Supp} \chi_{2}, \\
& \chi_{4} \equiv 1 \text { on a neighborhood of } \operatorname{Supp} \chi_{3},
\end{aligned}
$$

and Supp $\chi_{4} \subset \mathcal{V}_{\mathbf{c}}^{0} \subset \partial \Omega \times \Sigma_{+}$. These cut-off functions are introduced in order to use the localized Gårding's inequality (Theorem A.4). They are extended as homogeneous mappings of degree 0 with respect to $(\eta, \gamma)$. We write

$$
T_{\chi_{2} \beta Q_{0}^{-1}}^{\gamma} w(0)=T_{\chi_{2} \beta Q_{\text {in }}}^{\gamma} w_{\text {in }}(0)+T_{\chi_{2} \beta Q_{\text {out }}}^{\gamma} w_{\text {out }}(0) .
$$

Using the definition $w=T_{\chi Q}^{\gamma} \dot{v}$, we obtain

$$
T_{\chi_{2} \beta Q_{0}^{-1}}^{\gamma} w(0)=T_{\chi}^{\gamma} G+R^{\gamma} \dot{v}(0),
$$

where $R^{\gamma}$ is an operator of order $\leqslant-1$. We thus get

$$
\left\|T_{\chi_{2} \beta Q_{\text {in }}}^{\gamma} w_{\text {in }}(0)\right\|_{1, \gamma} \lesssim\|G\|_{1, \gamma}+\left\|w_{\text {out }}(0)\right\|_{1, \gamma}+\|\dot{v}(0)\|_{0} .
$$

We are now going to introduce the basis of the stable subspace in which $\boldsymbol{\beta}$ has a reduced expression. Because $\chi_{4} \chi_{2} \equiv \chi_{2}$, we have

$$
\chi_{2} \boldsymbol{\beta} Q_{\text {in }}=\left(\chi_{4} P_{1}^{-1}\right) \underbrace{\left(\chi_{2} P_{1} \boldsymbol{\beta} Q_{\text {in }} P_{2}\right)}_{\chi_{2} \boldsymbol{\beta}_{\text {in }}}\left(\chi_{4} P_{2}^{-1}\right) .
$$

We therefore obtain

$$
T_{\chi_{2} \beta Q_{\text {in }}}^{\gamma} w_{\text {in }}(0)=T_{\chi_{4} P_{1}^{-1}}^{\gamma} T_{\chi_{2} \beta_{\text {in }}}^{\gamma} T_{\chi_{4} P_{2}^{-1}}^{\gamma} w_{\text {in }}(0)+R^{\gamma} w_{\text {in }}(0),
$$

and this yields the inequality

$$
\left\|T_{\chi_{4} P_{1}^{-1}}^{\gamma} T_{\chi_{2} \beta_{\text {in }}}^{\gamma} T_{\chi_{4} P_{2}^{-1}}^{\gamma} w_{\text {in }}(0)\right\|_{1, \gamma} \lesssim\|G\|_{1, \gamma}+\left\|w_{\text {out }}(0)\right\|_{1, \gamma}+\|\dot{v}(0)\|_{0} .
$$

We first show that we have an estimate of the type

$$
\left\|T_{\chi_{4} P_{1}^{-1}}^{\gamma} T_{\chi_{2} \beta_{\text {in }}}^{\gamma} T_{\chi_{4} P_{2}^{-1}}^{\gamma} w_{\text {in }}(0)\right\|_{1, \gamma} \geqslant c\left\|T_{\chi_{2} \beta_{\text {in }}}^{\gamma} T_{\chi_{4} P_{2}^{-1}}^{\gamma} w_{\text {in }}(0)\right\|_{1, \gamma}-C\left\|w_{\text {in }}(0)\right\|_{0} .
$$

Define

$$
W:=T_{\chi_{2} \boldsymbol{\beta}_{\text {in }}}^{\gamma} T_{\chi_{4} P_{2}^{-1}}^{\gamma} w_{\text {in }}(0) .
$$

We first check that

$$
\left\|T_{\chi_{4} P_{1}^{-1}}^{\gamma} W\right\|_{1, \gamma}=\left\|\Lambda^{1, \gamma} T_{\chi_{4} P_{1}^{-1}}^{\gamma} W\right\|_{0} \geqslant\left\|T_{\chi_{4} P_{1}^{-1}}^{\gamma} \Lambda^{1, \gamma} W\right\|_{0}-C\left\|w_{\text {in }}(0)\right\|_{0} .
$$


We also note that

$$
T_{\chi_{3}}^{\gamma} \Lambda^{1, \gamma} W=\Lambda^{1, \gamma} W+R_{0}^{\gamma} W
$$

where $R_{0}^{\gamma}$ is of order $\leqslant 0$. We thus get

$$
\left\|T_{\chi_{4} P_{1}^{-1}}^{\gamma} W\right\|_{1, \gamma} \geqslant\left\|T_{\chi_{4} P_{1}^{-1}}^{\gamma} T_{\chi_{3}}^{\gamma} \Lambda^{1, \gamma} W\right\|_{0}-C\left\|w_{\text {in }}(0)\right\|_{0} .
$$

We are now going to use the ellipticity of $\left(P_{1}^{-1}\right)^{*} P_{1}^{-1}$ on the support of $\chi_{4}$. We write

$$
\begin{aligned}
\left\|T_{\chi_{4} P_{1}^{-1}}^{\gamma} T_{\chi_{3}}^{\gamma} \Lambda^{1, \gamma} W\right\|_{0}^{2} & =\left\langle\left(T_{\chi_{4} P_{1}^{-1}}^{\gamma}\right)^{*} T_{\chi_{4} P_{1}^{-1}}^{\gamma} T_{\chi_{3}}^{\gamma} \Lambda^{1, \gamma} W, T_{\chi_{3}}^{\gamma} \Lambda^{1, \gamma} W\right\rangle \\
& \geqslant \operatorname{Re}\left\langle T_{\chi_{4}^{2}\left(P_{1}^{-1}\right)^{*} P_{1}^{-1}}^{\gamma} T_{\chi_{3}}^{\gamma} \Lambda^{1, \gamma} W, T_{\chi_{3}}^{\gamma} \Lambda^{1, \gamma} W\right\rangle-C\left\|T_{\chi_{3}}^{\gamma} \Lambda^{1, \gamma} W\right\|_{0}\left\|T_{\chi_{3}}^{\gamma} \Lambda^{1, \gamma} W\right\|_{-1, \gamma},
\end{aligned}
$$

and we now apply Gårding's inequality (Theorem A.4) to obtain

$$
\left\|T_{\chi_{4} P_{1}^{-1}}^{\gamma} T_{\chi_{3}}^{\gamma} \Lambda^{1, \gamma} W\right\|_{0}^{2} \geqslant c\left\|T_{\chi_{3}}^{\gamma} \Lambda^{1, \gamma} W\right\|_{0}^{2}-C\left\|w_{\text {in }}(0)\right\|_{0}^{2} \geqslant c\left\|\Lambda^{1, \gamma} W\right\|_{0}^{2}-C\left\|w_{\text {in }}(0)\right\|_{0}^{2} .
$$

Plugging this later inequality in (55) yields

$$
\left\|\Lambda^{1, \gamma} T_{\chi_{2} \beta_{\text {in }}}^{\gamma} T_{\chi_{4} P_{2}^{-1}}^{\gamma} w_{\text {in }}(0)\right\|_{0} \lesssim\|G\|_{1, \gamma}+\left\|w_{\text {out }}(0)\right\|_{1, \gamma}+\|\dot{v}(0)\|_{0} .
$$

Observe that the difference

$$
\Lambda^{1, \gamma} T_{\chi_{2} \beta_{\text {in }}}^{\gamma}-T_{\chi_{2} \lambda^{1, \gamma} \boldsymbol{\beta}_{\text {in }}}^{\gamma}
$$

is of order $\leqslant 0$ so (56) also reads

$$
\left\|T_{\chi_{2} \lambda 1, \gamma}^{\gamma} \boldsymbol{\beta}_{\text {in }} T_{\chi_{4} P_{2}^{-1}}^{\gamma} w_{\text {in }}(0)\right\|_{0} \lesssim\|G\|_{1, \gamma}+\left\|w_{\text {out }}(0)\right\|_{1, \gamma}+\|\dot{v}(0)\|_{0} .
$$

It is time to use the particular structure of $\boldsymbol{\beta}_{\text {in }}$ to derive a lower bound for the left-hand term in (57). Recall that

$$
\chi_{2} \lambda^{1, \gamma} \boldsymbol{\beta}_{\text {in }}=\left(\begin{array}{cc}
\chi_{2}(\gamma+\mathrm{i} \sigma) & 0 \\
0 & \chi_{2} \lambda^{1, \gamma} I_{N-2}
\end{array}\right) .
$$

The relation $\chi \chi_{1} \equiv \chi$ yields

$$
T_{\chi_{4} P_{2}^{-1}}^{\gamma} w_{\text {in }}(0)=T_{\chi_{1}}^{\gamma} T_{\chi_{4} P_{2}^{-1}}^{\gamma} w_{\text {in }}(0)+R^{\gamma} w_{\text {in }}(0),
$$

so we rewrite (57) as

$$
\left\|T_{\chi_{2} \lambda^{1, \gamma} \boldsymbol{\beta}_{\text {in }}}^{\gamma} T_{\chi_{1}}^{\gamma} T_{\chi_{4} P_{2}^{-1}}^{\gamma} w_{\text {in }}(0)\right\|_{0} \lesssim\|G\|_{1, \gamma}+\left\|w_{\text {out }}(0)\right\|_{1, \gamma}+\|\dot{v}(0)\|_{0} .
$$

The decomposition

$$
T_{\chi_{4} P_{2}^{-1}}^{\gamma} w_{\text {in }}(0):=\left(\begin{array}{l}
W_{1} \\
W^{\prime}
\end{array}\right), \quad W_{1} \in \mathbb{C}, W^{\prime} \in \mathbb{C}^{N-2},
$$

gives

$$
\left\|T_{\chi_{2} \lambda^{1, \gamma} \boldsymbol{\beta}_{\text {in }}}^{\gamma} T_{\chi_{1}}^{\gamma} T_{\chi_{4} P_{2}^{-1}}^{\gamma} w_{\text {in }}(0)\right\|_{0}^{2}=\left\|T_{\chi_{2}(\gamma+i \sigma)}^{\gamma} T_{\chi_{1}}^{\gamma} W_{1}\right\|_{0}^{2}+\left\|T_{\chi_{2} \lambda^{1, \gamma}}^{\gamma} T_{\chi_{1}}^{\gamma} W^{\prime}\right\|_{0}^{2} .
$$

Because $\chi_{2} \chi_{1} \equiv \chi_{1}$ and $\chi_{1} \chi \equiv \chi$, we obtain

$$
\left\|T_{\chi_{2} \lambda 1, \gamma}^{\gamma} T_{\chi_{1}}^{\gamma} W^{\prime}\right\|_{0} \geqslant c\left\|W^{\prime}\right\|_{1, \gamma}-C\left\|w_{\text {in }}(0)\right\|_{0} \geqslant c\left\|T_{\sigma}^{\gamma} W^{\prime}\right\|_{0}-C\left\|w_{\text {in }}(0)\right\|_{0},
$$

and using that $\sigma \in \mathbb{R}$, we also obtain

$$
\left\|T_{\chi_{2}(\gamma+\mathrm{i} \sigma)}^{\gamma} T_{\chi_{1}}^{\gamma} W_{1}\right\|_{0} \geqslant c\left(\gamma\left\|W_{1}\right\|_{0}+\left\|T_{\sigma}^{\gamma} W_{1}\right\|_{0}\right)-C\left\|w_{\text {in }}(0)\right\|_{0} .
$$


Eventually, we get the lower-bound

$$
\left\|T_{\chi_{2} \lambda^{1, \gamma} \boldsymbol{\beta}_{\text {in }}}^{\gamma} T_{\chi_{1}}^{\gamma} T_{\chi_{4} P_{2}^{-1}}^{\gamma} w_{\text {in }}(0)\right\|_{0} \geqslant c\left(\gamma\left\|T_{\chi_{4} P_{2}^{-1}}^{\gamma} w_{\text {in }}(0)\right\|_{0}+\left\|T_{\sigma}^{\gamma} T_{\chi_{4} P_{2}^{-1}}^{\gamma} w_{\text {in }}(0)\right\|_{0}\right)-C\|\dot{v}(0)\|_{0} .
$$

To conclude, we perform the same kind of calculations as those already done with $\chi_{4} P_{1}^{-1}$ to show that

$$
\begin{aligned}
& \left\|T_{\chi_{4} P_{2}^{-1}}^{\gamma} w_{\text {in }}(0)\right\|_{0} \geqslant c\left\|w_{\text {in }}(0)\right\|_{0}-C\|\dot{v}(0)\|_{-1, \gamma}, \\
& \left\|T_{\sigma}^{\gamma} T_{\chi_{4} P_{2}^{-1}}^{\gamma} w_{\text {in }}(0)\right\|_{0} \geqslant c\left\|T_{\sigma}^{\gamma} w_{\text {in }}(0)\right\|_{0}-C\|\dot{v}(0)\|_{0} .
\end{aligned}
$$

The boundary terms thus satisfy:

$$
\gamma^{2}\left\|w_{\text {in }}(0)\right\|_{0}^{2}+\left\|T_{\sigma}^{\gamma} w_{\text {in }}(0)\right\|_{0}^{2} \lesssim\|G\|_{1, \gamma}^{2}+\left\|w_{\text {out }}(0)\right\|_{1, \gamma}^{2}+\|\dot{v}(0)\|_{0}^{2} .
$$

Combining (53) and (60), we obtain our main energy estimate localized near the instability points:

$$
\begin{aligned}
& \gamma\left\|w_{\text {out }}\right\|_{1, \gamma}^{2}+\gamma^{3}\left\|w_{\text {in }}\right\|_{0}^{2}+\sum_{\text {incoming }} \gamma\left\|T_{\sigma_{j}}^{\gamma} w_{j}\right\|_{0}^{2}+\left\|w_{\text {out }}(0)\right\|_{1, \gamma}^{2}+\gamma^{2}\left\|w_{\text {in }}(0)\right\|_{0}^{2}+\left\|T_{\sigma}^{\gamma} w_{\text {in }}(0)\right\|_{0}^{2} \\
& \lesssim\|G\|_{1, \gamma}^{2}+\|\dot{v}(0)\|_{0}^{2}+\frac{1}{\gamma}\left\|T_{\chi Q}^{\gamma} F\right\|_{1, \gamma}^{2}+\frac{1}{\gamma}\left(\|\dot{v}\|_{0}^{2}+\left\|T_{r}^{\gamma} \dot{v}\right\|_{1, \gamma}^{2}\right) .
\end{aligned}
$$

\subsection{Energy estimates far from instability points}

In this section, we show how to estimate the $L^{2}\left(H^{1}\right)$ norm of $\dot{v}$ far from the instability points. We fix a smooth cut-off function $\tilde{\chi}$ such that

$$
\tilde{\chi} \equiv 1 \quad \text { on } \operatorname{Supp} \chi_{0} \cap\left\{x_{d}=0\right\},
$$

so we have $\tilde{\chi} \chi_{\left.0\right|_{x_{d}=0}} \equiv \chi_{\left.0\right|_{x_{d}=0}}$, and we also ask that the support of $\tilde{\chi}$ does not meet the set of critical points $\Sigma_{\mathbf{c}}$. This is possible because the support of $\chi_{\left.0\right|_{x_{d}=0}}$ does not meet $\Sigma_{\mathbf{c}}$. With this requirement, the uniform stability condition is met at all point in the support of $\tilde{\chi}$. The cut-off function $\tilde{\chi}$ is introduced in order to use a localized Gårding's inequality.

To derive the desired energy estimate, we shall use a classical Kreiss' symmetrizer that is microlocalized around uniformly stable points.

Proposition 3.1 (Kreiss' symmetrizers). There exists a mapping

$$
S: \bar{\Omega} \times\left(\mathbb{R}^{d} \times \mathbb{R}^{+} \backslash\{0\}\right) \rightarrow \mathcal{M}_{2 n}(\mathbb{C})
$$

satisfying the following properties:

$\forall z$, the matrix $S(z)$ is Hermitian,

$S$ is a symbol of degree 2 and regularity 2 ,

$\forall z \in \partial \Omega \times\left(\mathbb{R}^{d} \times \mathbb{R}^{+} \backslash\{0\}\right)$, one has

$$
\tilde{\chi}(z)^{2} S(z)+C \tilde{\chi}(z)^{2} \lambda^{2, \gamma}(\eta) \boldsymbol{\beta}(z)^{*} \boldsymbol{\beta}(z) \geqslant c \tilde{\chi}(z)^{2} \lambda^{2, \gamma}(\eta) I,
$$

there exists a finite set of matrix valued mappings such that

$$
\operatorname{Re}(S(z) \mathbf{A}(z))=\sum_{l} V_{l}(z)^{*}\left(\begin{array}{cc}
\gamma H_{l}(z) & 0 \\
0 & E_{l}(z)
\end{array}\right) V_{l}(z),
$$


where $V_{l}$ and $E_{l}$ are homogeneous of degree 1 with respect to $(\eta, \gamma)$ (and belong to $\Gamma_{2}^{1}$ ), $H_{l}$ is homogeneous of degree 0 with respect to $(\eta, \gamma)$ (and belongs to $\Gamma_{2}^{0}$ ), and the following inequalities hold:

$$
\sum_{l} V_{l}(z)^{*} V_{l}(z) \geqslant c \lambda^{2, \gamma}(\eta) I, \quad H_{l}(z) \geqslant c I, \quad E_{l}(z) \geqslant c \lambda^{1, \gamma}(\eta) I
$$

We define $w:=T_{\chi_{0}}^{\gamma} \dot{v}$ and compute the equation satisfied by $w$. The calculations are entirely similar to those done in the preceeding paragraph, namely

$$
\partial_{d} w=T_{\mathbf{A}}^{\gamma} w+T_{\mathbf{A}_{d}^{-1}}^{\gamma} \mathbf{C} w+T_{r^{0}}^{\gamma} \dot{v}+R^{\gamma} \dot{v}+T_{\chi Q}^{\gamma} F,
$$

with

$$
r^{0}:=\partial_{d} \chi_{0}+\frac{1}{\mathrm{i}}\left\{\chi_{0}, \mathbf{A}\right\}=\partial_{d} \chi_{0}+\frac{1}{\mathrm{i}} \sum_{k=0}^{d-1}\left(\partial_{\eta_{k}} \chi_{0}\right) \partial_{x_{k}} \mathbf{A}-\left(\partial_{\eta_{k}} \mathbf{A}\right) \partial_{x_{k}} \chi_{0} .
$$

Let $\left\{\mathbf{S}^{\gamma}\left(x_{d}\right)\right\}$ be given by

$$
\mathbf{S}^{\gamma}\left(x_{d}\right):=\frac{1}{2}\left(\left(T_{S\left(x_{d}\right)}^{\gamma}\right)^{*}+T_{S\left(x_{d}\right)}^{\gamma}\right) .
$$

Because $S \in \Gamma_{2}^{2},\left\{\mathbf{S}^{\gamma}\right\}$ is a bounded Lipschitzean family of selfadjoint operators from $L^{2}\left(\mathbb{R}^{+} ; H^{2}\left(\mathbb{R}^{d}\right)\right)$ to $L^{2}(\Omega)$ (the bounds are uniform with respect to the parameter $\gamma \geqslant 1$ ). The starting point to derive the energy estimate is to take the scalar product of (64) with $\mathbf{S}^{\gamma} w$ and integrate with respect to $\left(x_{0}, \ldots, x_{d}\right) \in \Omega$. We find

$$
\begin{aligned}
& \left\langle\mathbf{S}^{\gamma}(0) w(0), w(0)\right\rangle_{L^{2}(\omega)}+2 \operatorname{Re}\left\langle\left\langle\mathbf{S}^{\gamma} T_{\mathbf{A}}^{\gamma} w, w\right\rangle_{L^{2}(\Omega)}\right. \\
& =-\operatorname{Re}\left\langle\left\langle\frac{\mathrm{d} \mathbf{S}^{\gamma}}{\mathrm{d} x_{d}} w, w\right\rangle\right\rangle_{L^{2}(\Omega)}-2 \operatorname{Re}\left\langle\left\langle\mathbf{S}^{\gamma} T_{\mathbf{A}_{d}^{-1} \mathbf{C}^{\gamma}}^{\gamma} w, w\right\rangle_{L^{2}(\Omega)}-2 \operatorname{Re}\left\langle\left\langle\mathbf{S}^{\gamma} T_{r^{0}}^{\gamma} \dot{v}, w \|_{L^{2}(\Omega)}\right.\right.\right. \\
& \left.\quad-2 \operatorname{Re} \| \mathbf{S}^{\gamma} R^{\gamma} \dot{v}, w\right\rangle_{L^{2}(\Omega)}-2 \operatorname{Re}\left\langle\left\langle\mathbf{S}^{\gamma} T_{\chi_{0}}^{\gamma} F, w \|_{L^{2}(\Omega)} .\right.\right.
\end{aligned}
$$

The right-hand side of (66) is easily estimated. We write $\mathbf{S}^{\gamma}$ as

$$
\mathbf{S}^{\gamma}=\Lambda^{1, \gamma} \Lambda^{-1, \gamma} \mathbf{S}^{\gamma}
$$

and use that $\Lambda^{1, \gamma}$ is selfadjoint. Because $\Lambda^{-1, \gamma} \mathbf{S}^{\gamma}$ is of order $\leqslant 1$, we obtain

$$
\begin{aligned}
& -\operatorname{Re}\left\langle\left\langle\frac{\mathrm{d} \mathbf{S}^{\gamma}}{\mathrm{d} x_{d}} w, w\right\rangle\right\rangle_{L^{2}(\Omega)} \leqslant C\|w\|_{1, \gamma}^{2}, \\
& -2 \operatorname{Re}\left\|\mathbf{S}^{\gamma} T_{\mathbf{A}_{d}^{-1} \mathbf{C}}^{\gamma} w, w\right\|_{L^{2}(\Omega)} \leqslant C\|w\|_{1, \gamma}^{2}, \\
& -2 \operatorname{Re}\left\|\mathbf{S}^{\gamma} T_{r^{0}}^{\gamma} \dot{v}, w\right\|_{L^{2}(\Omega)} \leqslant \frac{C}{\gamma}\left\|T_{r^{0}}^{\gamma} \dot{v}\right\|_{1, \gamma}^{2}+\varepsilon \gamma\|w\|_{1, \gamma}^{2}, \\
& -2 \operatorname{Re}\left\|\mathbf{S}^{\gamma} R^{\gamma} \dot{v}, w\right\|_{L^{2}(\Omega)} \leqslant \frac{C}{\gamma}\|\dot{v}\|_{0}^{2}+\varepsilon \gamma\|w\|_{1, \gamma}^{2}, \\
& -2 \operatorname{Re}\left\|\mathbf{S}^{\gamma} T_{\chi_{0}}^{\gamma} F, w\right\|_{L^{2}(\Omega)} \leqslant \frac{C}{\gamma}\left\|T_{\chi_{0}}^{\gamma} F\right\|_{1, \gamma}^{2}+\varepsilon \gamma\|w\|_{1, \gamma}^{2},
\end{aligned}
$$

so we get

$$
\begin{aligned}
& \left\langle\mathbf{S}^{\gamma}(0) w(0), w(0)\right\rangle_{L^{2}(\omega)}+2 \operatorname{Re}\left\langle\| \mathbf{S}^{\gamma} T_{\mathbf{A}}^{\gamma} w, w\right\rangle_{L^{2}(\Omega)} \\
& \quad \leqslant(C+3 \varepsilon \gamma)\|w\|_{1, \gamma}^{2}+\frac{C}{\gamma}\left\|T_{\chi_{0}}^{\gamma} F\right\|_{1, \gamma}^{2}+\frac{C}{\gamma}\left(\|\dot{v}\|_{0}^{2}+\left\|T_{r^{0}}^{\gamma} \dot{v}\right\|_{1, \gamma}^{2}\right) .
\end{aligned}
$$


We are now going to derive a lower bound for the left-hand side of (67) by means of Gårding's inequalities. We first deal with the boundary term. First note that

$$
\mathbf{S}(0)-T_{S(0)}^{\gamma}
$$

is of order $\leqslant 1$, so we have

$$
\left\langle\mathbf{S}^{\gamma}(0) w(0), w(0)\right\rangle_{L^{2}(\omega)}=\operatorname{Re}\left\langle T_{S(0)}^{\gamma}(0) w(0), w(0)\right\rangle_{L^{2}(\omega)}+\mathcal{O}\left(\|w(0)\|_{1, \gamma}\|w(0)\|_{0}\right) .
$$

Using (62), we can apply Gårding's inequality (Theorem A.4) and derive

$$
\operatorname{Re}\left\langle T_{S(0)}^{\gamma}(0) w(0), w(0)\right\rangle_{L^{2}(\omega)}+C \operatorname{Re}\left\langle T_{\lambda^{2, \gamma} \boldsymbol{\beta}^{*} \boldsymbol{\beta}}^{\gamma} w(0), w(0)\right\rangle_{L^{2}(\omega)} \geqslant c\|w(0)\|_{1, \gamma}^{2}-C\|\dot{v}(0)\|_{0}^{2} .
$$

Observe that

$$
T_{\lambda^{2, \gamma} \boldsymbol{\beta}^{*} \boldsymbol{\beta}}^{\gamma}-\left[\Lambda^{1, \gamma} T_{\boldsymbol{\beta}}^{\gamma}\right]^{*}\left[\Lambda^{1, \gamma} T_{\boldsymbol{\beta}}^{\gamma}\right]
$$

is of order $\leqslant 1$ so we have

$$
\operatorname{Re}\left\langle T_{\lambda^{2, \gamma} \boldsymbol{\beta}^{*} \boldsymbol{\beta}}^{\gamma} w(0), w(0)\right\rangle_{L^{2}(\omega)}=\left\|T_{\boldsymbol{\beta}}^{\gamma} w(0)\right\|_{1, \gamma}^{2}+\mathcal{O}\left(\|w(0)\|_{1, \gamma}\|w(0)\|_{0}\right) .
$$

For $\gamma$ large enough, we therefore obtain

$$
\left\langle\mathbf{S}^{\gamma}(0) w(0), w(0)\right\rangle_{L^{2}(\omega)} \geqslant c\|w(0)\|_{1, \gamma}^{2}-C\left\|T_{\boldsymbol{\beta}}^{\gamma} w(0)\right\|_{1, \gamma}^{2}-C\|\dot{v}(0)\|_{0}^{2},
$$

and we now deal with the interior term. Since

$$
\operatorname{Re} \mathbf{S}^{\gamma} T_{\mathbf{A}}^{\gamma}-T_{\operatorname{Re}(S \mathbf{A})}^{\gamma}
$$

is of order $\leqslant 2$, we have

$$
2 \operatorname{Re}\left\langle\left\langle\mathbf{S}^{\gamma} T_{\mathbf{A}}^{\gamma} w, w\right\rangle_{L^{2}(\Omega)} \geqslant 2 \operatorname{Re}\left\langle T_{\operatorname{Re}(S \mathbf{A})}^{\gamma} w, w\left\|_{L^{2}(\Omega)}-C\right\| w \|_{1, \gamma}^{2} .\right.\right.
$$

Define

$$
\forall z \in \bar{\Omega} \times\left(\mathbb{R}^{d} \times \mathbb{R}^{+} \backslash\{0\}\right), \quad a_{i}(z):=\left(\begin{array}{cc}
\gamma H_{i}(z) & 0 \\
0 & E_{i}(z)
\end{array}\right) .
$$

Then the remainders

$$
T_{V_{l}^{*} a_{l} V_{l}}^{\gamma}-\left(T_{V_{l}}^{\gamma}\right)^{*} T_{a_{l}}^{\gamma} T_{V_{l}}^{\gamma}
$$

are of order $\leqslant 2$, so we get

$$
2 \operatorname{Re}\left\langle\left\langle\mathbf{S}^{\gamma} T_{\mathbf{A}}^{\gamma} w, w\left\|_{L^{2}(\Omega)} \geqslant \operatorname{Re} \sum_{l}\right\| T_{a_{l}}^{\gamma} w_{l}, w_{l}\left\|_{L^{2}(\Omega)}-C\right\| w \|_{1, \gamma}^{2},\right.\right.
$$

where $w_{l}:=T_{V_{l}}^{\gamma} w$. Using the block decomposition of $a_{i}$ and Gårding's inequality (Theorem A.3) on each block, we obtain

$$
\operatorname{Re}\left\langle\left\langle T_{a_{l}}^{\gamma} w_{l}, w_{l}\left\|_{L^{2}(\Omega)} \geqslant c \gamma\right\| w_{l} \|_{0}^{2},\right.\right.
$$

and the ellipticity of the symbol $\sum_{l} V_{l}^{*} V_{l}$ yields, for $\gamma$ large enough, the estimate

$$
\|w\|_{1, \gamma}^{2} \lesssim \sum_{l} \operatorname{Re}\left\langle T_{V_{l}^{*} V_{l}}^{\gamma} w, w\left\|_{L^{2}(\Omega)} \lesssim \sum_{l}\right\| w_{l}\left\|_{0}^{2}+\frac{1}{\gamma}\right\| w \|_{1, \gamma}^{2} .\right.
$$

Combining all these inequalities, we conclude that

$$
\operatorname{Re}\left\langle\left\langle\mathbf{S}^{\gamma} T_{\mathbf{A}}^{\gamma} w, w\right\rangle_{L^{2}(\Omega)} \geqslant c \gamma\|w\|_{1, \gamma}^{2}\right.
$$


for $\gamma$ large enough. We now use (68) and (69) to derive a lower bound for the left-hand term of (67). Choosing $\varepsilon$ appropriately, we end up with

$$
\gamma\|w\|_{1, \gamma}^{2}+\|w(0)\|_{1, \gamma}^{2}-\left\|T_{\beta}^{\gamma} w(0)\right\|_{1, \gamma}^{2}-\|\dot{v}(0)\|_{0}^{2} \lesssim \frac{1}{\gamma}\left\|T_{\chi_{0}}^{\gamma} F\right\|_{1, \gamma}^{2}+\frac{1}{\gamma}\left(\|\dot{v}\|_{0}^{2}+\|\| T_{r^{0}}^{\gamma} \dot{v} \|_{1, \gamma}^{2}\right) .
$$

To conclude, observe that the remainder

$$
T_{\boldsymbol{\beta}}^{\gamma} T_{\chi_{0}}^{\gamma}-T_{\chi_{0}}^{\gamma} T_{\boldsymbol{\beta}}^{\gamma}
$$

is of order $\leqslant-1$ so we have

$$
\left\|T_{\boldsymbol{\beta}}^{\gamma} w(0)\right\|_{1, \gamma}^{2} \lesssim\|G\|_{1, \gamma}^{2}+\|\dot{v}(0)\|_{0}^{2} .
$$

Eventually, we have proved

$$
\gamma\left\|T_{\chi_{0}}^{\gamma} \dot{v}\right\|_{1, \gamma}^{2}+\left\|T_{\chi_{0}}^{\gamma} \dot{v}(0)\right\|_{1, \gamma}^{2} \lesssim\|G\|_{1, \gamma}^{2}+\|\dot{v}(0)\|_{0}^{2}+\frac{1}{\gamma}\left\|T_{\chi_{0}}^{\gamma} F\right\|_{1, \gamma}^{2}+\frac{1}{\gamma}\left(\|\dot{v}\|_{0}^{2}+\|\| T_{r^{0}}^{\gamma} \dot{v} \|_{1, \gamma}^{2}\right) .
$$

\subsection{The main result}

This paragraph is devoted to the very end of the analysis. We first prove the following:

Theorem 3.1. For all $\dot{v} \in H^{1}(\Omega)$, we define the localized norm of $\dot{v}$ as

$$
\left\|\dot{v} \tilde{\|}^{2}:=\right\| T_{\chi_{0}}^{\gamma} \dot{v}\left\|_{1, \gamma}^{2}+\right\| \dot{v}_{\text {out }}\left\|_{1, \gamma}^{2}+\gamma^{2}\right\| \dot{v}_{\text {in }}\left\|_{0}^{2}+\sum_{1 \leqslant j \leqslant J^{\prime}}\right\| T_{\sigma_{j}}^{\gamma} \dot{v}_{j} \|_{0}^{2},
$$

where notations are similar to those of Section 3.4:

$$
T_{\chi Q}^{\gamma} \dot{v}=\left(\begin{array}{c}
\dot{v}_{\text {in }} \\
\dot{v}_{\text {out }}
\end{array}\right), \quad \dot{v}_{\text {in }} \in \mathbb{C}^{N-1}, \dot{v}_{\text {out }} \in \mathbb{C}^{N+1} \text { and } \dot{v}_{\text {in }}=\left(\begin{array}{c}
\dot{v}_{1} \\
\vdots \\
\dot{v}_{J^{\prime}}
\end{array}\right) \text {. }
$$

For $\dot{v}(0) \in H^{1}\left(\mathbb{R}^{d}\right)$ we define in a similar way the localized norm of $\dot{v}(0)$ as

$$
\|\dot{v}(0)\|^{2}:=\left\|T_{\chi 0}^{\gamma} \dot{v}(0)\right\|_{1, \gamma}^{2}+\left\|\dot{v}_{\text {out }}(0)\right\|_{1, \gamma}^{2}+\gamma^{2}\left\|\dot{v}_{\text {in }}(0)\right\|_{0}^{2}+\left\|T_{\sigma}^{\gamma} \dot{v}_{\text {in }}(0)\right\|_{0}^{2} \text {. }
$$

Then there exist two constants $C>0$ and $\gamma_{0} \geqslant 1$ such that for all $\gamma \geqslant \gamma_{0}$ and for all $(v, \psi) \in H^{2}(\Omega) \times H^{2}\left(\mathbb{R}^{d}\right)$, the following estimate holds:

$$
\gamma\left\|\dot{v} \tilde{\|}^{2}+\right\| \dot{v}(0) \tilde{\|}^{2} \leqslant C\left(\frac{1}{\gamma}\|F\|_{1, \gamma}^{2}+\|G\|_{1, \gamma}^{2}\right)
$$

where

$$
\dot{v}:=v-T_{\partial_{d} \mathbf{a}}^{\gamma} \psi, \quad F:=\partial_{d} \dot{v}-T_{\mathbf{A}}^{\gamma} \dot{v}-T_{\mathbf{A}_{d}^{-1} \mathbf{C}}^{\gamma} \dot{v} \quad \text { and } \quad G:=T_{\beta}^{\gamma} \dot{v}_{\left.\right|_{d}=0} .
$$

Proof. Using (61) and (70), we have already proved that there exists two constants $C$ and $\gamma_{0}$ such that for all $\gamma \geqslant \gamma_{0}$, one has

$$
\gamma\left\|\dot{v} \tilde{\|}^{2}+\right\| \dot{v}(0) \tilde{\|}^{2} \leqslant C\left(\frac{1}{\gamma}\|F\|_{1, \gamma}^{2}+\|G\|_{1, \gamma}^{2}\right)+C\|\dot{v}(0)\|_{0}^{2}+\frac{C}{\gamma}\left(\|\dot{v}\|_{0}^{2}+\|\| T_{r}^{\gamma} \dot{v}\left\|_{0}^{2}+\right\| T_{r^{0}}^{\gamma} \dot{v} \|_{0}^{2}\right),
$$

where $r$ is given by (43) and $r^{0}$ is given by (65). We first show that the localized norm verifies

$$
\gamma\|\dot{v}\|_{0} \leqslant C\|\dot{v}\|
$$


for $\gamma$ large enough and $C>0$. Write

$$
I=\chi_{0} I+\chi I=\chi_{0} I+\left(\tilde{\chi} Q_{0}^{-1}\right) \chi Q_{0},
$$

where $\tilde{\chi}$ is a suitable cut-off function such that $\tilde{\chi} \chi=\chi$. Then we have

$$
\begin{aligned}
\gamma\|\dot{v}\|_{0} & \leqslant \gamma\left\|T_{\chi_{0}}^{\gamma} \dot{v}\right\|_{0}+C \gamma\left\|T_{\chi Q_{0}}^{\gamma} \dot{v}\right\|_{0} \leqslant\left\|T_{\chi_{0}}^{\gamma} \dot{v}\right\|_{1, \gamma}+C \gamma\left\|T_{\chi Q}^{\gamma} \dot{v}\right\|_{0}+C \gamma\left\|T_{\chi Q_{-1}}^{\gamma} \dot{v}\right\|_{0} \\
& \leqslant\left\|T_{\chi_{0}}^{\gamma} \dot{v}\right\|_{1, \gamma}+\left\|\dot{v}_{\text {out }}\right\|\left\|_{1, \gamma}+\gamma\right\| \dot{v}_{\text {in }}\left\|_{0}+C\right\| \dot{v}\left\|_{0} \leqslant C\right\| \dot{v}\|+C\| \dot{v} \|_{0}
\end{aligned}
$$

and this gives (73) when $\gamma$ is large enough. In a completely similar way, we get

$$
\gamma\|\dot{v}(0)\|_{0} \leqslant C \| \dot{v}(0) \tilde{\|}
$$

Using (73) and (74), (72) yields

$$
\gamma\left\|\dot{v} \tilde{\|}^{2}+\right\| \dot{v}(0) \tilde{\|}^{2} \leqslant C\left(\frac{1}{\gamma}\|F\|_{1, \gamma}^{2}+\|G\|_{1, \gamma}^{2}\right)+\frac{C}{\gamma}\left(\left\|T_{r}^{\gamma} \dot{v}\right\|_{1, \gamma}^{2}+\left\|T_{r^{0}}^{\gamma} \dot{v}\right\|_{1, \gamma}^{2}\right),
$$

and we need to absorb the last two error terms on the right-hand side.

To absorb the term $T_{r}^{\gamma} \dot{v}$, we decompose the symbol $r$ as a linear combination of $\chi_{0}$ and the $\sigma_{j}$ 's. Recall that $r$ is a symbol of degree 0 and regularity 1 that is identically zero in the region $\{\chi \equiv 1\}$. In the region $\{\chi \leqslant 1 / 2\}$, we have $\chi_{0} \geqslant 1 / 2$ so we can write

$$
r:=\alpha_{0}\left(\chi_{0} I\right)
$$

with $\alpha$ a symbol of degree 0 . In the region $\{1>\chi \geqslant 1 / 2\}$, we can write

$$
r:=\left(\begin{array}{c}
\alpha_{1} \\
\vdots \\
\alpha_{J^{\prime}} \\
\alpha_{\text {out }}
\end{array}\right)\left(\begin{array}{cccc}
\sigma_{1} I_{n_{1}} & & & \\
& \ddots & & \\
& & \sigma_{J^{\prime}} I_{n_{J^{\prime}}} & \\
& & & I_{N+1}
\end{array}\right)\left(\chi Q_{0}\right),
$$

because the two last matrices are regular in the region where $r$ is not identically zero (this is because $\left|\sigma_{j}\right| \geqslant c$ when $r$ is not zero). Up to introducing new cut-off functions, we can decompose $r$ as

$$
r=\alpha_{0}\left(\chi_{0} I\right)+\left(\begin{array}{c}
\alpha_{1} \\
\vdots \\
\alpha_{J^{\prime}} \\
\alpha_{\text {out }}
\end{array}\right)\left(\begin{array}{cccc}
\sigma_{1} I_{n_{1}} & & \\
& \ddots & & \\
& & \sigma_{J^{\prime}} I_{n_{J^{\prime}}} & \\
& & & I
\end{array}\right)\left(\chi Q_{0}\right),
$$

where $\alpha_{0}$ and $\alpha_{\text {out }}$ are of degree 0 and $\alpha_{1}, \ldots, \alpha_{J^{\prime}}$ are of degree -1 (because the $\sigma_{j}$ 's are of degree 1 ). Using the above decomposition, we easily derive

$$
\left\|T_{r}^{\gamma} \dot{v}\right\|_{1, \gamma} \lesssim\left\|T_{\chi_{0}}^{\gamma} \dot{v}\right\|_{1, \gamma}+\left\|\dot{v}_{\text {out }}\right\|\left\|_{1, \gamma}+\sum_{1 \leqslant j \leqslant J^{\prime}}\right\| T_{\sigma_{j}}^{\gamma} \dot{v}_{j}\left\|_{0} \lesssim\right\| \dot{v} \tilde{\|} .
$$

The same kind of arguments also work for the term $T_{r^{0}}^{\gamma}$ because $r^{0}$ is identically zero near the bicharacteristic curves. Eventually, (75) yields, for $\gamma$ large enough:

$$
\gamma\left\|\dot{v} \tilde{\|}^{2}+\right\| \dot{v}(0) \tilde{\|}^{2} \lesssim \frac{1}{\gamma}\|F\|_{1, \gamma}^{2}+\|G\|_{1, \gamma}^{2} .
$$


Theorem 3.1 gives a precise statement of the location of the possible singularities of $\dot{v}$, that is, where $\dot{v}$ is less regular than the source terms $F$ and $G$. However, an important consequence of Theorem 3.1 is that our constant coefficients energy estimate holds for variable coefficients system:

Theorem 3.2. Let $\mathbf{a}$, defined by (24), satisfy Assumptions 6-8. Then for all $v \in H^{2}(\Omega), \psi \in H^{2}\left(\mathbb{R}^{d}\right)$ and for all $\gamma \geqslant \gamma_{0}$, one has

$$
\gamma\|v\|_{0}^{2}+\left\|v_{\left.\right|_{x_{d}=0}}\right\|_{0}^{2}+\|\psi\|_{1, \gamma}^{2} \lesssim \frac{1}{\gamma^{3}}\left\|\mathcal{L}_{\mathbf{a}}^{\gamma}(v, \psi)\right\|_{1, \gamma}^{2}+\frac{1}{\gamma^{2}}\left\|\mathcal{B}_{\mathbf{a}}^{\gamma}(v, \psi)\right\|_{1, \gamma}^{2} .
$$

Proof. The result is now a direct consequence of the paralinearization estimates. Let $v$ and $\psi$ be given and define $\dot{v}$ as the good unknown of the problem. Let

$$
F:=\partial_{d} \dot{v}-T_{\mathbf{A}}^{\gamma} \dot{v}-T_{\mathbf{A}_{d}^{-1} \mathbf{C}}^{\gamma} \dot{v} \quad \text { and } \quad G:=T_{\beta}^{\gamma} \dot{v}_{\left.\right|_{d}=0} .
$$

Using (76) and (73), (74), we get

$$
\gamma\|\dot{v}\|_{0}^{2}+\|\dot{v}(0)\|_{0}^{2} \lesssim \frac{1}{\gamma^{3}}\|F\|_{1, \gamma}^{2}+\frac{1}{\gamma^{2}}\|G\|_{1, \gamma}^{2} .
$$

We know from (36) that $G$ satisfies

$$
\|G\|_{1, \gamma}^{2} \lesssim\|\dot{v}(0)\|_{0}^{2}+\left\|T_{\mathbf{b}}^{\gamma} \psi+T_{\mathbf{M}}^{\gamma} \dot{v}_{x_{d}=0}\right\|_{1, \gamma}^{2}+\|\psi\|_{1, \gamma}^{2},
$$

so we have

$$
\gamma\|\dot{v}\|_{0}^{2}+\|\dot{v}(0)\|_{0}^{2} \lesssim \frac{1}{\gamma^{3}}\|F\|_{1, \gamma}^{2}+\frac{1}{\gamma^{2}}\left\|T_{\mathbf{b}}^{\gamma} \psi+T_{\mathbf{M}}^{\gamma} \dot{v}_{x_{d}=0}\right\|_{1, \gamma}^{2}+\frac{1}{\gamma^{2}}\|\psi\|_{1, \gamma}^{2} .
$$

Using (33), we obtain

$$
\gamma\|\dot{v}\|_{0}^{2}+\|\dot{v}(0)\|_{0}^{2} \lesssim \frac{1}{\gamma^{3}}\|F\|_{1, \gamma}^{2}+\frac{1}{\gamma^{2}}\left\|\mathcal{B}_{\mathbf{a}}^{\gamma}(v, \psi)\right\|_{1, \gamma}^{2}+\frac{1}{\gamma^{2}}\|\psi\|_{1, \gamma}^{2},
$$

and using once again (33) (for the term $\|F\|_{1, \gamma}$ ) yields

$$
\gamma\|\dot{v}\|_{0}^{2}+\|\dot{v}(0)\|_{0}^{2} \lesssim \frac{1}{\gamma^{3}}\left\|\mathcal{L}_{\mathbf{a}}^{\gamma}(v, \psi)\right\|_{1, \gamma}^{2}+\frac{1}{\gamma^{2}}\left\|\mathcal{B}_{\mathbf{a}}^{\gamma}(v, \psi)\right\|_{1, \gamma}^{2}+\frac{1}{\gamma^{2}}\|\psi\|_{1, \gamma}^{2} .
$$

Estimate (34) yields the estimate for $v$ (and not $\dot{v}$ ) while (35) enables us to recover the estimate on the shock front $\psi$ and to derive (77).

\section{The example of gas dynamics}

When dealing with a concrete example, it is more convenient to have distinct notations for the Laplace variable and the Fourier variable. We shall thus denote by $\tau$ the Laplace dual variable of $t$ ( $\tau$ is a complex number of positive real part) and by $\eta \in \mathbb{R}^{d-1}$ the Fourier dual variable of $\left(x_{1}, \ldots, x_{d-1}\right)$. Consequently, the stable subspace will be denoted by $\mathcal{E}^{-}(\tau, \eta)$, the front symbol will be denoted by $b(\tau, \eta)$ and so on.

Consider Euler's equations of isentropic gas dynamics:

$$
\left\{\begin{array}{l}
\partial_{t} \rho+\nabla \cdot(\rho \mathbf{u})=0, \\
\partial_{t}(\rho \mathbf{u})+\nabla \cdot(\rho \mathbf{u} \otimes \mathbf{u})+\nabla p=0,
\end{array}\right.
$$

where $p$ is an increasing but nonconvex function of $\rho>0$. System (78) satisfies both Assumptions 1 and 2. For a planar shock wave

$$
U= \begin{cases}\left(\rho_{l}, \mathbf{u}_{l}\right) & \text { if } x \cdot v<\sigma t, \\ \left(\rho_{r}, \mathbf{u}_{r}\right) & \text { if } x \cdot v>\sigma t,\end{cases}
$$


where $v$ is a unit vector in $\mathbb{R}^{d}$, the Rankine-Hugoniot conditions read

$$
\begin{aligned}
& \rho_{r}\left(\mathbf{u}_{r} \cdot v-\sigma\right)=\rho_{l}\left(\mathbf{u}_{l} \cdot v-\sigma\right)=: j, \\
& j[\mathbf{u}]+[p] v=0,
\end{aligned}
$$

and we shall assume $j \neq 0$. Then the tangential velocity is continuous across the shock front and, up to changing observer, we may assume

$$
v=(0, \ldots, 0,1), \quad \sigma=0, \quad \mathbf{u}_{r, l}=\left(0, \ldots, 0, u_{r, l}\right), \quad j=\rho_{r} u_{r}=\rho_{l} u_{l}>0 .
$$

The Mach number on both sides of the shock front is defined by

$$
M_{r, l}:=\frac{u_{r, l}}{c_{r, l}} \quad \text { with } c_{r, l}:=\sqrt{p^{\prime}\left(\rho_{r, l}\right)} .
$$

As usual, $c$ denotes the sound speed in the fluid. One easily checks that $U$ is a 1-shock if and only if

$$
M_{l}>1, \quad M_{r}<1 .
$$

In such a case, the following result is proved in [24]:

Proposition 4.1 (Majda [24]). The shock $U$ is uniformly stable if and only if

$$
M_{r}^{2}\left(\frac{\rho_{r}}{\rho_{l}}-1\right)<1
$$

When $p$ is not a convex function of $\rho$, one may have

$$
M_{r}^{2}\left(\frac{\rho_{r}}{\rho_{l}}-1\right)>1
$$

In this case, the following is proved in [12]:

Proposition $4.2[12]$. There exists $V_{1}>0$ such that for all $(\tau, \eta) \in \mathbb{C} \times \mathbb{R}^{d-1}$ satisfying $\operatorname{Re} \tau \geqslant 0,(\tau, \eta) \neq(0,0)$ and $\tau \neq \pm \mathrm{i} V_{1}|\eta|$, one has

$$
\left\{(Z, \chi) \in \mathcal{E}^{-}(\tau, \eta) \times \mathbb{C} \text { s.t. } \chi b(\tau, \eta)+M Z=0\right\}=\{0\},
$$

and for $\eta \neq 0$, the set

$$
\left\{(Z, \chi) \in \mathcal{E}^{-}\left( \pm \mathrm{i} V_{1}|\eta|, \eta\right) \times \mathbb{C} \text { s.t. } \chi b\left( \pm \mathrm{i} V_{1}|\eta|, \eta\right)+M Z=0\right\}
$$

is a one-dimensional subspace of $\mathbb{C}^{2 d+3}$.

By definition, $V_{1}^{2}$ is the smallest root of the polynomial

$$
P_{1}(X):=\left(c_{r}^{2}-u_{r}^{2}\right)\left(X^{2}+u_{r}^{2} u_{l}^{2}\right)+\left[4 u_{r}^{2} c_{r}^{2}-2 u_{r} u_{l}\left(c_{r}^{2}+u_{r}^{2}\right)\right] X,
$$

that has two distinct positive roots (the greatest is denoted $V_{2}^{2}$ ). Furthermore we have

$$
c_{r}^{2}-u_{r}^{2}<V_{1}^{2}<u_{r} u_{l} \frac{c_{r}^{2}-u_{r}^{2}}{c_{r}^{2}+u_{r}^{2}}<V_{2}^{2} .
$$

We recall a few results of [12]. The eigenvalues $\omega_{j}$ 's of the symbol $\mathcal{A}(U, \tau, \eta)$ are $\omega_{2}^{r}:=-\tau / u_{r}, \omega_{2}^{l}:=\tau / u_{l}$ and the roots $\omega_{1,3}^{r}, \omega_{1,3}^{l}$ of the polynomial equations:

$$
\begin{aligned}
& \left(\tau+u_{r} \omega\right)^{2}=c_{r}^{2}\left(\omega^{2}-|\eta|^{2}\right), \\
& \left(\tau-u_{l} \omega\right)^{2}=c_{l}^{2}\left(\omega^{2}-|\eta|^{2}\right) .
\end{aligned}
$$


We choose $\omega_{3}^{r}$ as the root of negative real part of (80b) when $\operatorname{Re} \tau>0$. We also define $a_{3}^{r}:=\tau u_{r}-\left(c_{r}^{2}-u_{r}^{2}\right) \omega_{3}^{r}$.

It is proved in [12] that Assumptions 4 and 5 hold as long as (79) is satisfied. Therefore, all planar shock waves satisfying (79) are weakly stable in the sense of Definition 2. Moreover, in a suitable neighborhood of the critical frequencies $\Sigma_{\mathrm{cr}}(\mathbf{u})$, there exists a $\mathcal{C}^{\infty}$ basis (that we write under the form of a rectangular matrix $Q_{\text {in }}$ ) of the stable subspace $\mathcal{E}^{-}$such that

$$
\beta(\tau, \eta) Q_{\text {in }}=\left(\begin{array}{cc}
\rho_{r}\left(c_{r}^{2} \tau+u_{r} a_{3}^{r}\right) & 2 i j \eta^{t} \\
i j \eta\left(c_{r}^{2} \tau+u_{l} a_{3}^{r}\right) & -\rho_{r}\left(\tau^{2} I_{d-1}+u_{r} u_{l} \eta \otimes \eta\right)
\end{array}\right) .
$$

Using simple calculations on matrices, we are going to show that Assumption 8 holds. Let us first look at the 2-dimensional case: $\eta$ is a real number and $\beta Q_{\text {in }}$ is a $2 \times 2$ matrix.

For all complex numbers $\xi_{1}, \xi_{2}, \xi_{3}, \xi_{4}, \xi_{5}$ such that $\xi_{4} \neq 0$ and $\xi_{5} \neq 0$, the identity

$$
\left(\begin{array}{cc}
1 / \xi_{5} & -\xi_{2} /\left(\xi_{4} \xi_{5}\right) \\
0 & 1 / \xi_{4}
\end{array}\right)\left(\begin{array}{ll}
\xi_{1} & \xi_{2} \\
\xi_{3} & \xi_{4}
\end{array}\right)\left(\begin{array}{cc}
\xi_{4} & 0 \\
-\xi_{3} & 1
\end{array}\right)=\left(\begin{array}{cc}
\left(\xi_{1} \xi_{4}-\xi_{2} \xi_{3}\right) / \xi_{5} & 0 \\
0 & 1
\end{array}\right)
$$

is a straightforward calculation. If we write (81) under the form

$$
\beta(\tau, \eta) Q_{\text {in }}=\left(\begin{array}{ll}
\xi_{1} & \xi_{2} \\
\xi_{3} & \xi_{4}
\end{array}\right)
$$

we can easily check that $\xi_{4}$ does not vanish in the neighborhood of the critical frequencies. In the neighborhood of $\left( \pm \mathrm{i} V_{1}|\eta|, \eta\right) \in \Sigma_{+}$, the determinant of $\beta(\tau, \eta) Q_{\text {in }}$ reads

$$
\xi_{1} \xi_{4}-\xi_{2} \xi_{3}=\left(\tau-\mathrm{i} V_{1}|\eta|\right) h(\tau, \eta) \quad \text { or } \quad \xi_{1} \xi_{4}-\xi_{2} \xi_{3}=\left(\tau+\mathrm{i} V_{1}|\eta|\right) h(\tau, \eta)
$$

for a suitable $\mathcal{C}^{\infty}$ function $h$ that does not vanish, see [12]. Setting $\xi_{5}:=h(\tau, \eta)$, we obtain two regular matrices $P_{1}(\tau, \eta)$ and $P_{2}(\tau, \eta)$ such that

$$
P_{1}(\tau, \eta) \beta(\tau, \eta) Q_{\text {in }} P_{2}(\tau, \eta)=\left(\begin{array}{cc}
\tau \pm \mathrm{i} V_{1}|\eta| & 0 \\
0 & 1
\end{array}\right) .
$$

This is nothing but Assumption 8 since the set of critical frequencies is precisely defined by $\tau= \pm \mathrm{i} V_{1}|\eta|$. In this case, the set of critical frequencies $\Sigma_{\mathrm{cr}}(U)$ has exactly four connected components and we have a real equation of each of them.

In space dimension 3, the computations are similar. Observe that the matrix $\tau^{2} I_{d-1}+u_{r} u_{l} \eta \otimes \eta$ is regular (near the critical frequencies) according to Proposition 4.2. Hence $\beta Q_{\text {in }}$ reads

$$
\beta Q_{\mathrm{in}}=\left(\begin{array}{lll}
\xi_{1} & \xi_{2} & \xi_{3} \\
\xi_{4} & \xi_{5} & \xi_{6} \\
\xi_{7} & \xi_{8} & \xi_{9}
\end{array}\right)=\left(\begin{array}{cc}
\xi_{1} & \ell \\
* & \Xi
\end{array}\right)
$$

where $\Xi:=\left(\tau^{2} I_{d-1}+u_{r} u_{l} \eta \otimes \eta\right)$. One has $\operatorname{det} \Xi \neq 0$ near the critical frequencies. We check the identity

$$
\left(\begin{array}{cc}
1 & -\ell \Xi^{-1} \\
0 & \Xi^{-1}
\end{array}\right)\left(\begin{array}{lll}
\xi_{1} & \xi_{2} & \xi_{3} \\
\xi_{4} & \xi_{5} & \xi_{6} \\
\xi_{7} & \xi_{8} & \xi_{9}
\end{array}\right)\left(\begin{array}{lll}
\xi_{5} \xi_{9}-\xi_{6} \xi_{8} & 0 & 0 \\
\xi_{6} \xi_{7}-\xi_{4} \xi_{9} & 1 & 0 \\
\xi_{4} \xi_{8}-\xi_{5} \xi_{7} & 0 & 1
\end{array}\right)=\left(\begin{array}{ccc}
\operatorname{det}\left(\beta Q_{\text {in }}\right) & 0 & 0 \\
0 & 1 & 0 \\
0 & 0 & 1
\end{array}\right)
$$

and we can conclude that Assumption 8 is also satisfied in the 3-dimensional case.

\section{Acknowledgements}

I warmly thank Sylvie Benzoni-Gavage and Guy Métivier for stimulating discussions and precious help. I also thank the referee for valuable comments that deeply improved the original version of this article. 


\section{Appendix. Paradifferential calculus with a parameter}

In this appendix, we collect the main results of the paradifferential calculus of Bony and Meyer $[10,28]$ that we use in this paper. The introduction of a positive parameter was achieved by Mokrane [29], see also [27]. We refer to these papers for the proofs of the results stated below. We first recall the classification of paradifferential symbols:

Definition 3. A paradifferential symbol of degree $m \in \mathbb{R}$ and regularity $k(k \in \mathbb{N})$ is a function $a(x, \xi, \gamma): \mathbb{R}^{d} \times$ $\mathbb{R}^{d} \times\left[0,+\infty\left[\rightarrow \mathbb{C}^{N \times N}\right.\right.$ such that $a$ is $\mathcal{C}^{\infty}$ with respect to $\xi$ and for all $\alpha \in \mathbb{N}^{d}$, there exists a constant $C_{\alpha}$ verifying

$$
\forall(\xi, \gamma), \quad\left\|\partial_{\xi}^{\alpha} a(\cdot, \xi, \gamma)\right\|_{W^{k, \infty}} \leqslant C_{\alpha} \lambda^{m-|\alpha|, \gamma}(\xi)=C_{\alpha}\left(\gamma^{2}+|\xi|^{2}\right)^{(m-|\alpha|) / 2} .
$$

The set of paradifferential symbols of degree $m$ and regularity $k$ is denoted by $\Gamma_{k}^{m}$. We denote by $\Sigma_{k}^{m}$ the subset of paradifferential symbols $a \in \Gamma_{k}^{m}$ such that for a suitable $\left.\varepsilon \in\right] 0,1[$ one has

$$
\forall(\xi, \gamma), \quad \operatorname{Supp} \mathcal{F}_{x} a(\cdot, \xi, \gamma) \subset\left\{\eta \in \mathbb{R}^{d}|| \eta \mid \leqslant \varepsilon\left(\gamma^{2}+|\xi|^{2}\right)^{1 / 2}\right\} .
$$

Of course, the symbols in $\Sigma_{k}^{m}$ are $\mathcal{C}^{\infty}$ functions with respect to both variables $x$ and $\xi$, and for all $a \in \Sigma_{k}^{m}$, we have the estimates

$$
\forall(x, \xi, \gamma), \quad\left|\partial_{x}^{\beta} \partial_{\xi}^{\alpha} a(x, \xi, \gamma)\right| \leqslant C_{\alpha, \beta} \lambda^{m-|\alpha|+|\beta|, \gamma}(\xi) .
$$

Thus any symbol $a \in \Sigma_{k}^{m}$ belongs to Hörmander's class $S_{1,1}^{m}[19]$ and defines an operator $P^{\gamma}(a)$ on the Schwartz' class $\mathcal{S}$ by the usual formula

$$
\forall u \in \mathcal{S}, \quad P^{\gamma}(a) u(x):=\frac{1}{(2 \pi)^{d}} \int_{\mathbb{R}^{d}} \mathrm{e}^{\mathrm{i} x \cdot \xi} a(x, \xi, \gamma) \hat{u}(\xi) \mathrm{d} \xi .
$$

We shall use the following terminology:

Definition 4. A family of operators $\left\{P^{\gamma}\right\}$ defined for $\gamma \geqslant 1$ will be said of order $\leqslant m(m \in \mathbb{R})$ if the operators $P^{\gamma}$ are uniformly bounded from $H_{\gamma}^{s+m}$ to $H_{\gamma}^{s}$ :

$$
\forall \gamma \geqslant 1, \forall u \in H_{\gamma}^{s+m}, \quad\left\|P^{\gamma} u\right\|_{s, \gamma} \leqslant C(s, m)\|u\|_{s+m, \gamma} .
$$

The following theorem is crucial for the sequel of the analysis:

Theorem A.1. If $a \in \Sigma_{k}^{m}$, the family $\left\{P^{\gamma}(a)\right\}$ is of order $\leqslant m$.

The regularization of symbols in the class $\Gamma_{k}^{m}$ is achieved by a convolution with admissible cut-off functions:

Definition 5. Let $\psi: \mathbb{R}^{d} \times \mathbb{R}^{d} \times\left[1,+\infty\left[\rightarrow\left[0,+\infty\left[\right.\right.\right.\right.$ be a $\mathcal{C}^{\infty}$ function such that the following estimates hold for all $\alpha, \beta \in \mathbb{N}^{d}$ :

$$
\forall(\eta, \xi, \gamma), \quad\left|\partial_{\eta}^{\alpha} \partial_{\xi}^{\beta} \psi(\eta, \xi, \gamma)\right| \leqslant C_{\alpha, \beta} \lambda^{-|\alpha|-|\beta|, \gamma}(\xi) .
$$

We shall say that $\psi$ is an admissible cut-off function if there exist real numbers $0<\varepsilon_{1}<\varepsilon_{2}<1$ satisfying

$$
\begin{array}{ll}
\psi(\eta, \xi, \gamma)=1 & \text { if }|\eta| \leqslant \varepsilon_{1}\left(\gamma^{2}+|\xi|^{2}\right)^{1 / 2}, \\
\psi(\eta, \xi, \gamma)=0 & \text { if }|\eta| \geqslant \varepsilon_{2}\left(\gamma^{2}+|\xi|^{2}\right)^{1 / 2}
\end{array}
$$

An example of cut-off function is the following: let $\chi$ be a nonnegative $\mathcal{C}^{\infty}$ function on $\mathbb{R}^{d} \times \mathbb{R}$ such that 


$$
\begin{aligned}
& \gamma_{1}^{2}+\left|\xi_{1}\right|^{2} \geqslant \gamma_{2}^{2}+\left|\xi_{2}\right|^{2} \Longrightarrow \chi\left(\xi_{1}, \gamma_{1}\right) \leqslant \chi\left(\xi_{2}, \gamma_{2}\right), \\
& \begin{cases}\chi(\xi, \gamma)=1 & \text { if }\left(\gamma^{2}+|\xi|^{2}\right)^{1 / 2} \leqslant 1 / 2, \\
\chi(\xi, \gamma)=0 & \text { if }\left(\gamma^{2}+|\xi|^{2}\right)^{1 / 2} \geqslant 1 .\end{cases}
\end{aligned}
$$

We define a function $\varphi(\xi, \gamma):=\chi(\xi / 2, \gamma / 2)-\chi(\xi, \gamma)$. Then the function $\psi_{0}$ defined by

$$
\psi_{0}(\eta, \xi, \gamma):=\sum_{p \geqslant 0} \chi\left(2^{2-p} \eta, 0\right) \varphi\left(2^{-p} \xi, 2^{-p} \gamma\right)
$$

is an admissible cut-off function (one can take $\varepsilon_{1}=1 / 16$ and $\varepsilon_{2}=1 / 2$ ).

If $\psi$ is an admissible cut-off function, the inverse Fourier transform $K^{\psi}$ of $\psi(\cdot, \xi, \gamma)$ satisfies

$$
\forall(\xi, \gamma), \quad\left\|\partial_{\xi}^{\alpha} K^{\psi}(\cdot, \xi, \gamma)\right\|_{L^{1}} \leqslant C_{\alpha} \lambda^{-|\alpha|, \gamma}(\xi) .
$$

These $L^{1}$ bounds on $\partial_{\xi}^{\alpha} K^{\psi}$ enable us to establish the following proposition:

Proposition A.1. Let $\psi$ be an admissible cut-off function. The mapping

$$
a \mapsto \sigma_{a}^{\psi}(x, \xi, \gamma):=\int_{\mathbb{R}^{d}} K^{\psi}(x-y, \xi, \gamma) a(y, \xi, \gamma) \mathrm{d} y
$$

is continuous from $\Gamma_{k}^{m}$ to $\Sigma_{k}^{m}$ for all $m$.

If $a \in \Gamma_{1}^{m}$, then $a-\sigma_{a}^{\psi} \in \Gamma_{0}^{m-1}$. In particular, if $\psi_{1}$ and $\psi_{2}$ are two admissible cut-off functions and $a \in \Gamma_{1}^{m}$, then $\sigma_{a}^{\psi_{1}}-\sigma_{a}^{\psi_{2}} \in \Sigma_{0}^{m-1}$.

Fixing an admissible cut-off function $\psi$, we define the paradifferential operator $T_{a}^{\psi, \gamma}$ by the formula

$$
T_{a}^{\psi, \gamma}:=P^{\gamma}\left(\sigma_{a}^{\psi}\right)
$$

If $\psi_{1}$ and $\psi_{2}$ are two admissible cut-off functions and $a \in \Gamma_{1}^{m}$, then Proposition A.1 and Theorem A.1 show that the family $\left\{T_{a}^{\psi_{1}, \gamma}-T_{a}^{\psi_{2}, \gamma}\right\}$ is of order $\leqslant(m-1)$.

The symbolic calculus is based on the following theorem:

Theorem A.2. Let $a \in \Gamma_{1}^{m}$ and $b \in \Gamma_{1}^{m^{\prime}}$. Then $a b \in \Gamma_{1}^{m+m^{\prime}}$ and the family

$$
\left\{T_{a}^{\psi, \gamma} \circ T_{b}^{\psi, \gamma}-T_{a b}^{\psi, \gamma}\right\}_{\gamma \geqslant 1}
$$

is of order $\leqslant m+m^{\prime}-1$ for all admissible cut-off function $\psi$.

Let $a \in \Gamma_{1}^{m}$. Then for all admissible cut-off function $\psi$, the family

$$
\left\{\left(T_{a}^{\psi, \gamma}\right)^{*}-T_{a^{*}}^{\psi, \gamma}\right\}_{\gamma \geqslant 1}
$$

is of order $\leqslant m-1$.

Let $a \in \Gamma_{2}^{m}$ and $b \in \Gamma_{2}^{m^{\prime}}$. Then $a b \in \Gamma_{2}^{m+m^{\prime}}$ and the family

$$
\left\{T_{a}^{\psi, \gamma} \circ T_{b}^{\psi, \gamma}-T_{a b}^{\psi, \gamma}-T_{-i \sum_{j} \partial_{\xi_{j}} a \partial_{x_{j}} b}^{\psi, \gamma}\right\}_{\gamma}
$$

is of order $\leqslant m+m^{\prime}-2$ for all admissible cut-off function $\psi$.

Let $a \in \Gamma_{2}^{m}$. Then the family

$$
\left\{\left(T_{a}^{\psi, \gamma}\right)^{*}-T_{a^{*}}^{\psi, \gamma}-T_{-i \sum_{j} \partial_{\xi_{j}} \partial_{x_{j}} a^{*}}^{\psi, \gamma}\right\}_{\gamma \geqslant 1}
$$

is of order $\leqslant m-2$ for all admissible cut-off function $\psi$. 
The next theorem is the parameter version of Gårding's inequality:

Theorem A.3. Let $a \in \Gamma_{1}^{2 m}$ and let $\psi$ be and admissible cut-off function. Assume that there exists a constant c $>0$ such that

$$
\forall(x, \xi, \gamma), \quad \operatorname{Re} a(x, \xi, \gamma) \geqslant c \lambda^{2 m, \gamma}(\xi) \operatorname{Id} .
$$

Then there exists $\gamma_{0} \geqslant 1$ such that

$$
\forall \gamma \geqslant \gamma_{0}, \forall u \in H_{\gamma}^{m}, \quad \operatorname{Re}\left\langle T_{a}^{\psi, \gamma} u, u\right\rangle_{H^{-m}, H^{m}} \geqslant \frac{c}{2}\|u\|_{m, \gamma}^{2} .
$$

We also have a microlocalized version of Gårding's inequality:

Theorem A.4. Let $a \in \Gamma_{1}^{2 m}, \chi \in \Gamma_{1}^{0}$ and $\psi$ be and admissible cut-off function. Assume that there exists $\tilde{\chi} \in \Gamma_{1}^{0}$ and a constant $c>0$ such that $\tilde{\chi} \geqslant 0, \tilde{\chi} \chi=\chi$ and

$$
\forall(x, \xi, \gamma), \quad \tilde{\chi}^{2}(x, \xi, \gamma) \operatorname{Re} a(x, \xi, \gamma) \geqslant c \tilde{\chi}^{2}(x, \xi, \gamma) \lambda^{2 m, \gamma}(\xi) I .
$$

Then there exists $\gamma_{0} \geqslant 1$ and $C>0$ such that

$$
\forall \gamma \geqslant \gamma_{0}, \quad \forall u \in H_{\gamma}^{m}\left(\mathbb{R}^{d}\right), \quad \operatorname{Re}\left\langle T_{a}^{\psi, \gamma} T_{\chi}^{\psi, \gamma} u, T_{\chi}^{\psi, \gamma} u\right\rangle_{H^{-m}, H^{m}} \geqslant \frac{c}{2}\left\|T_{\chi}^{\psi, \gamma} u\right\|_{m, \gamma}^{2}-C\|u\|_{m-1, \gamma}^{2} .
$$

We now study the case of paraproducts: they are defined by the particular choice of $\psi_{0}$ as cut-off function. We shall write $T_{a}^{\gamma}$ instead of $T_{a}^{\psi_{0}, \gamma}$ for the paradifferential operators obtained after smoothing by the function $\psi_{0}$. We have the following important result:

Theorem A.5. Let $a \in W^{1, \infty}\left(\mathbb{R}^{d}\right), u \in L^{2}\left(\mathbb{R}^{d}\right)$ and $\gamma \geqslant 1$. Then we have

$$
\begin{aligned}
& \left\|a u-T_{a}^{\gamma} u\right\|_{0} \leqslant \frac{C}{\gamma}\|a\|_{W^{1, \infty}}\|u\|_{0}, \\
& \left\|a \partial_{j} u-T_{a}^{\gamma}\left(\partial_{j} u\right)\right\|_{0} \leqslant C\|a\|_{W^{1, \infty}}\|u\|_{0},
\end{aligned}
$$

for a suitable constant $C$ that is independent of $(a, u, \gamma)$.

If $a \in W^{2, \infty}\left(\mathbb{R}^{d}\right)$, we have

$$
\begin{aligned}
& \left\|a u-T_{a}^{\gamma} u\right\|_{1, \gamma} \leqslant \frac{C}{\gamma}\|a\|_{W^{2, \infty}}\|u\|_{0}, \\
& \left\|a \partial_{j} u-T_{a}^{\gamma}\left(\partial_{j} u\right)\right\|_{1, \gamma} \leqslant C\|a\|_{W^{2, \infty}}\|u\|_{0},
\end{aligned}
$$

for a suitable constant $C$ that is independent of $(a, u, \gamma)$.

We can extend the paradifferential calculus to symbols defined on a half-space in the following way: we still denote by $\Gamma_{k}^{m}$ the set of symbols $a\left(x_{0}, \ldots, x_{d}, \eta, \gamma\right)$ defined on $\Omega \times\left(\mathbb{R}^{d} \times[0,+\infty[\backslash\{0\})\right.$ such that the mapping $x_{d} \mapsto a\left(\cdot, x_{d}, \cdot\right)$ is bounded into $\Gamma_{k}^{m}$. We define the paradifferential operator $T_{a}^{\gamma}$ by

$$
\forall u \in \mathcal{C}_{c}^{\infty}(\bar{\Omega}), \forall x_{d} \geqslant 0, \quad\left(T_{a}^{\gamma} u\right)\left(\cdot, x_{d}\right):=T_{a\left(x_{d}\right)}^{\gamma} u\left(\cdot, x_{d}\right) .
$$

Using Theorem A.5 and integrating with respect to $x_{d}$, we obtain for all symbol $a \in W^{1, \infty}(\Omega)$ and all $u \in L^{2}(\Omega)$ the estimates:

$$
\begin{aligned}
& \left\|a u-T_{a}^{\gamma} u\right\|_{0} \leqslant \frac{C}{\gamma}\|a\|_{W^{1, \infty}(\Omega)}\|u\|_{0}, \\
& \left\|a \partial_{j} u-T_{a}^{\gamma}\left(\partial_{j} u\right)\right\|_{0} \leqslant C\|a\|_{W^{1, \infty}(\Omega)}\|u\|_{0}, \quad 0 \leqslant j \leqslant d-1 .
\end{aligned}
$$




\section{References}

[1] R.A. Adams, Sobolev Spaces, Academic Press, 1975.

[2] S. Alinhac, Existence d'ondes de raréfaction pour des systèmes quasi-linéaires hyperboliques multidimensionnels, Comm. Partial Differential Equations 14 (2) (1989) 173-230.

[3] S. Alinhac, Blowup for Nonlinear Hyperbolic Equations, Birkhäuser, Boston, 1995.

[4] V.I. Arnol'd, Ordinary Differential Equations, Springer-Verlag, 1992.

[5] S. Benzoni-Gavage, Stability of multi-dimensional phase transitions in a van der Waals fluid, Nonlinear Anal. 31 (1-2) (1998) $243-263$.

[6] S. Benzoni-Gavage, Stability of subsonic planar phase boundaries in a van der Waals fluid, Arch. Rational Mech. Anal. 150 (1) (1999) $23-55$.

[7] S. Benzoni-Gavage, F. Rousset, D. Serre, K. Zumbrun, Generic types and transitions in hyperbolic initial-boundary value problems, Proc. Roy. Soc. Edinburgh Sect. A 132 (2002) 1073-1104.

[8] S. Benzoni-Gavage, D. Serre, First order systems of hyperbolic partial differential equations with applications, in preparation.

[9] A.M. Blokhin, Y.L. Trakhinin, Stability of fast parallel MHD shock waves in polytropic gas, Eur. J. Mech. B Fluids 18 (2) (1999) $197-211$.

[10] J.M. Bony, Calcul symbolique et propagation des singularités pour les équations aux dérivées partielles non linéaires, Ann. Sci. École Norm. Sup. (4) 14 (2) (1981) 209-246.

[11] J. Chazarain, A. Piriou, Introduction to the Theory of Linear Partial Differential Equations, North-Holland, 1982.

[12] J.F. Coulombel, Weak stability of nonuniformly stable multidimensional shocks, SIAM J. Math. Anal. 34 (1) (2002) $142-172$.

[13] C.M. Dafermos, Hyperbolic Conservation Laws in Continuum Physics, Springer-Verlag, 2000.

[14] J. Francheteau, G. Métivier, Existence de chocs faibles pour des systèmes quasi-linéaires hyperboliques multidimensionnels, in: Astérisque, vol. 268, 2000.

[15] H. Freistühler, Some results on the stability of non-classical shock waves, J. Partial Differential Equations 111 (1) (1998) 25-38.

[16] H. Freistühler, C. Fries, C. Rohde, Existence, bifurcation, and stability of profiles for classical and non-classical shock waves, in: Ergodic Theory, Analysis, and Efficient Simulation of Dynamical Systems, Springer, 2001, pp. 287-309, 814.

[17] R. Hersh, Mixed problems in several variables, J. Math. Mech. 12 (1963) 317-334.

[18] M.W. Hirsch, Differential Topology, Springer-Verlag, 1994.

[19] L. Hörmander, Lectures on Nonlinear Hyperbolic Differential Equations, Springer-Verlag, 1997.

[20] T. Kato, Perturbation Theory for Linear Operators, second ed., Springer-Verlag, 1976.

[21] H.O. Kreiss, Initial boundary value problems for hyperbolic systems, Comm. Pure Appl. Math. 23 (1970) 277-298.

[22] P.D. Lax, Hyperbolic systems of conservation laws. II, Comm. Pure Appl. Math. 10 (1957) 537-566.

[23] A. Majda, The existence of multi-dimensional shock fronts, Mem. Amer. Math. Soc. 43 (281) (1983).

[24] A. Majda, The stability of multi-dimensional shock fronts, Mem. Amer. Math. Soc. 41 (275) (1983).

[25] A. Majda, Compressible Fluid Flow and Systems of Conservation Laws in Several Space Variables, Springer-Verlag, 1984.

[26] G. Métivier, The block structure condition for symmetric hyperbolic systems, Bull. London Math. Soc. 32 (6) (2000) 689-702.

[27] G. Métivier, Stability of multidimensional shocks, in: Advances in the Theory of Shock Waves, Birkhäuser, 2001, pp. 25-103.

[28] Y. Meyer, Remarques sur un théorème de J.M. Bony, Suppl. Rend. Circ. Mat. Palermo Ser. II 1 (1981) 1-20.

[29] A. Mokrane, Problèmes mixtes hyperboliques non-linéaires, Ph.D. Thesis, Université de Rennes I, 1987.

[30] T. Ohkubo, T. Shirota, On structures of certain $L^{2}$-well-posed mixed problems for hyperbolic systems of first order, Hokkaido Math. J. 4 (1975) 82-158.

[31] J.V. Ralston, Note on a paper of Kreiss, Comm. Pure Appl. Math. 24 (6) (1971) 759-762.

[32] M. Sablé-Tougeron, Existence pour un problème de l'élastodynamique Neumann non linéaire en dimension 2, Arch. Rational Mech. Anal. 101 (3) (1988) 261-292.

[33] D. Serre, Systems of Conservation Laws. 1, Cambridge University Press, 1999.

[34] D. Serre, Systems of Conservation Laws. 2, Cambridge University Press, 2000.

[35] D. Serre, La transition vers l'instabilité pour les ondes de choc multi-dimensionnelles, Trans. Amer. Math. Soc. 353 (12) (2001) $5071-$ 5093. 